DNA Library of Life, research article

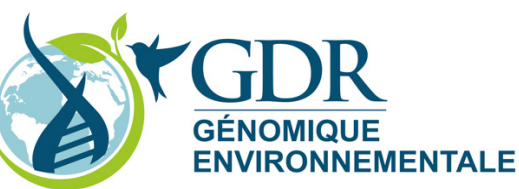

urn:1sid:zoobank.org:pub:FC663FAD-BCCB-4423-8952-87E93B14DEEA

\title{
Review of the Nassarius pauperus (Gould, 1850) complex (Nassariidae): Part 3, reinstatement of the genus Reticunassa, with the description of six new species
}

\author{
Lee Ann GALINDO ${ }^{1 *}$, Hugo H. KOOL ${ }^{2} \&$ Henk DEKKER ${ }^{3}$ \\ ${ }^{1}$ Muséum national d'Histoire naturelle, Département Systématique et Evolution, ISyEB Institut \\ (UMR 7205 CNRS/UPMC/MNHN/EPHE), 43, Rue Cuvier, 75231 Paris, France. \\ ${ }^{2,3}$ Associate Mollusca Collection, Naturalis Biodiversity Center, \\ P.O. Box 9517, 2300 RA Leiden, the Netherlands. \\ *Corresponding author: galindo@mnhn.fr \\ ${ }^{2}$ Email: hugo.kool@naturalis.nl \\ ${ }^{3}$ Email: h-dekker@quicknet.nl \\ ${ }^{1}$ urn:Isid:zoobank.org:author:B84DC387-F1A5-4FE4-80F2-5C93E41CEC15 \\ ${ }^{2}$ urn:1sid:zoobank.org:author:5E718E5A-85C8-404C-84DC-6E53FD1D61D6 \\ ${ }^{3}$ urn:1sid:zoobank.org:author:DA6A1E69-F70A-42CC-A702-FE0EC80D77FA
}

\begin{abstract}
In this review (third part), several species within the Nassarius pauperus complex from the eastern Indian Ocean and western Pacific are treated, including a revised concept of Nassa paupera Gould, 1850, type species of the genus Reticunassa Iredale, 1936. In the most recent taxonomic revision, several species had been synonymized with Nassarius pauperus (Gould, 1850), despite distinctive differences among these species in shell morphology. We sequenced a fragment of the mitochondrial COI and the nuclear 28S genes of all available Nassarius pauperus complex species. Automatic barcode gap discovery and reciprocal monophyly were applied to propose species delimitation hypotheses and to support a new concept of the genus. Using morphological and molecular characters, Reticunassa is elevated to full genus rank. Six new species are described: Reticunassa visayaensis sp. nov., $R$. poppeorum sp. nov., $R$. annabolteae sp. nov., $R$. goliath sp. nov., $R$. intrudens sp. nov. and $R$. thailandensis sp. nov., while $R$. tringa (Souverbie, 1864) is recognized as a valid species.
\end{abstract}

Keywords. Nassarius pauperus complex, taxonomy, species delimitation, neotype, barcoding.

Galindo L.A., Kool H.H. \& Dekker H. 2017. Review of the Nassarius pauperus (Gould, 1850) complex (Nassariidae). Part 3, reinstatement of the genus Reticunassa, with the description of six new species. European Journal of Taxonomy 275: 1-43. http://dx.doi.org/10.5852/ejt.2017.275

\section{Introduction}

In his systematic revision of the species of Nassariidae, Cernohorsky (1984) treated 16 taxa as junior synonyms of Nassarius (Hinia) pauperus (Gould, 1850) (as "Hima"). All these nassariids are rather 
similar and the small morphological differences were interpreted by Cernohorsky (1984) as representing intraspecific variation. Kool \& Dekker $(2006,2007)$ discussed, in parts 1 and 2 of this ongoing review, whether many of these synonyms should not be considered valid species. DNA data obtained from live specimens collected during several MNHN expeditions were used here as evidence to support species hypotheses originally based solely on morphological features.

Kool \& Dekker $(2006,2007)$ defined some informative morphological characters for the species included in the Nassarius pauperus complex (often as "pauper-complex"). Among these characters are the type and sculpture of the protoconch, the morphology of the spiral cords, the sculpture on the surface between the spiral cords, and the shape and sculpture of the columellar callus. Using molecular characters, Galindo et al. (2016) demonstrated that species attributed to the Nassarius pauperus complex clustered together in a clade separate from the genus Nassarius Duméril, 1805 s. str.

In part 1 of the Nassarius pauperus complex review, Kool \& Dekker (2006) described six species with intercordal axial sculpture. In part 2, Kool \& Dekker (2007) treated four species with intercordal spiral sculpture, from the western Indian Ocean to the central Pacific Ocean. In this third review, we treat eight species (of which six are new to science) from the eastern Indian Ocean and the Western Pacific, all with intercordal spiral sculpture. The new taxonomic scheme is supported by a species tree based on molecular data.

On the basis of the molecular analysis presented here, Reticunassa Iredale, 1936 is elevated to full genus status to include the species formally considered as constituting the Nassarius pauperus complex.

\section{Material and methods}

Material was obtained from various natural history museums and private collections, listed in the acronyms section. Live-collected specimens used for molecular studies were anesthetized with $\mathrm{MgCl}_{2}$ or microwaved (see Galindo et al. 2014). The bodies, preserved in $95 \%$ ethanol, were removed from the shells in the laboratory to prevent progressive deterioration of the shells by etching. The shells are kept dry and have been assigned the same registration number as the corresponding body or tissue in ethanol.

A stereo microscope was used to observe shell morphology and color patterns. A digital caliper (accurate to $0.1 \mathrm{~mm}$ ) was used for measurements. Shells of the new species were photographed in dorsal, ventral and lateral views, using a Canon EOS 60D camera. Scanning electron microscope (SEM) images were obtained by backscattered electron diffraction on a VEGA II LSU TESCAN, under conventional high vacuum at $20 \mathrm{kV}$ acceleration voltage (12 to $15 \mathrm{~Pa}$ chamber pressure) at the SEM-EDS of the 'Direction des Collections' at MNHN. In general, the terms and methods used for the morphological analysis follow part 1 of the review (Kool \& Dekker 2006).

\section{Acronyms}

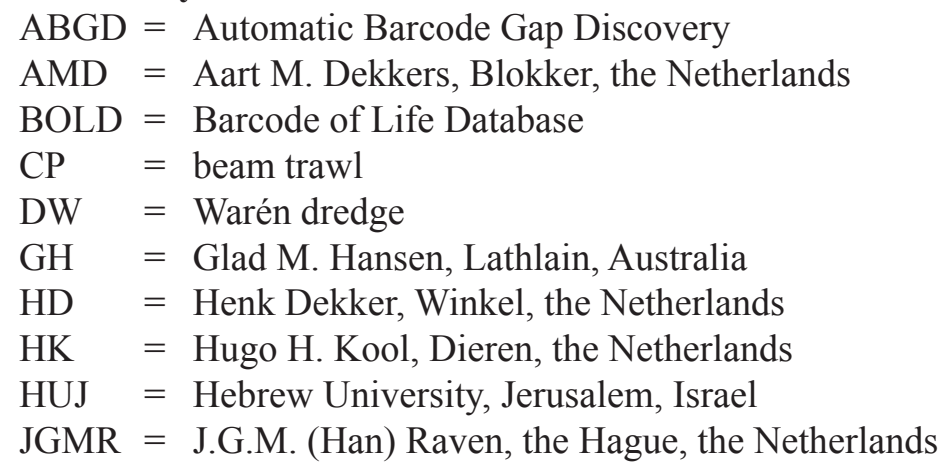



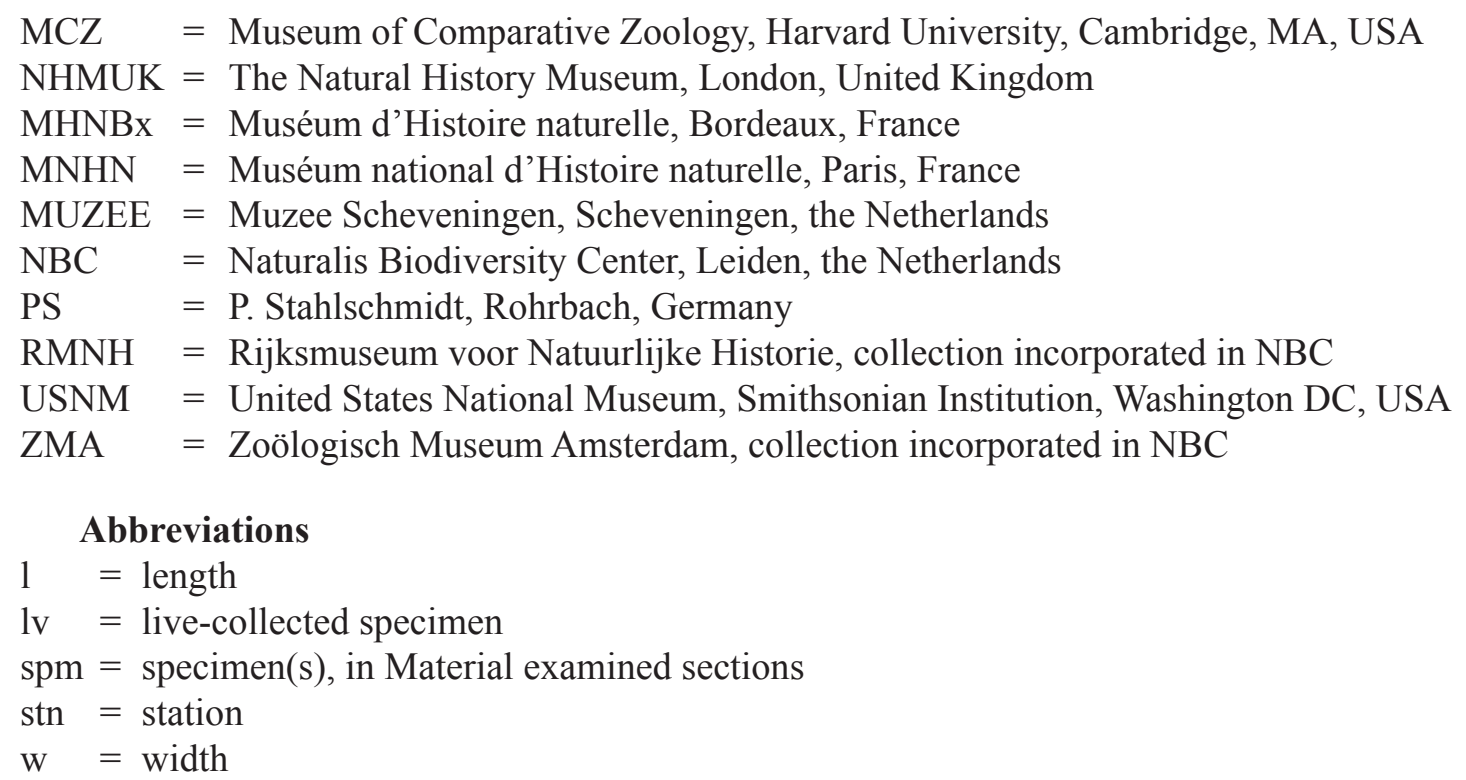

Molecular sequencing was carried out at the Service de Systématique Moléculaire (MNHN UMS 2700). Procedures from the Consortium for the Barcode of Life barcoding protocols (www.barcodeoflife.org) adapted to large biodiversity collections were followed (see Puillandre et al. 2012a). A piece of muscle from the foot, sufficient for approximately five DNA extractions, was stored in a 2D barcode tube as a reference tissue collection. Extraction was done with the NucleoSpin ${ }^{\circledR} 96$ Tissue Core Kit adapted to a NucleoSpin ${ }^{\circledR} 96$ Plasmid Binding Plate (Macherey-Nagel) using a liquid handling workstation (epMotion ${ }^{\circledR} 5075$ VAC, Eppendorf). Purified DNA was stored in a second 2D tube. PCR reactions were performed in $20 \mu \mathrm{l}$, containing $2 \mu \mathrm{l}$ of DNA, $1 \times$ reaction buffer, $2.5 \mathrm{mM} \mathrm{MgCl}_{2}, 0.26 \mathrm{mM}$ dNTP, $0.3 \mu \mathrm{M}$ of each primer, $1 \mu \mathrm{LMSO}, 1 \mu \mathrm{l} \mathrm{BSA}(10 \mathrm{mg} / \mathrm{l})$ and 1.5 units of Q-Bio Taq DNA polymerase (QBiogene, Carlsbad, California, USA). A fragment of $658 \mathrm{bp}$ of the cytochrome oxidase I (COI) mitochondrial gene was amplified, using the universal primer set (LCO1490 and HCO2198) (Folmer et al. 1994) and a primer set specific for Nassariidae (5COIF: 5'ACAAATCATAAAGAYATTGGAAC-3', 492COIR: 5'CGCTCAAATTGTATTCCTCG-3' and 492COIRD: 5'CGYTCRAATTGTATYCCTCG-3') (Galindo et al. 2016). Annealing temperature was $50^{\circ} \mathrm{C}$ for 35 seconds. The first subunit of the $28 \mathrm{~S}$ gene was amplified using the $\mathrm{C} 1$ ' and D2 primers of Chisholm et al. (2001). Annealing temperature was $58^{\circ} \mathrm{C}$ for 40 seconds or a touchdown gradient from $66^{\circ} \mathrm{C}$ to $56^{\circ} \mathrm{C}$ for 8 seconds (plus $58^{\circ} \mathrm{C}$ for 30 seconds) whenever double bands were present. Sequencing was carried out by the Centre National de Séquençage, Genoscope (Évry, France) and Eurofins Genomics (Paris, France). In all cases, both directions were sequenced to confirm the accuracy of each haplotype.

Sequence cleaning and alignment was done by using Codon Code Aligner software. GenBank accession numbers for all the sequences obtained are included in Table 1. We compiled a dataset of sequences available for several closely related species, identified in a phylogenetic tree, based on a large dataset of Nassariidae (Galindo et al. 2016) to compare with our new species hypotheses. The analysis involved 89 COI sequences: 72 of them belong to the Nassarius pauperus complex, 16 sequences to other species of Nassariidae and one to a distant outgroup. The Automatic Barcode Gap Discovery (ABGD) method using the default parameters (Puillandre et al. 2012b) was employed. It automatically detects, when present, a threshold (the barcode gap) in the pairwise distribution of the genetic distances calculated between each pair of specimens.

To test whether the species hypotheses proposed by ABGD corresponded to monophyletic groups, Bayesian analyses, consisting of two Markov chains (35 million generations each with a sampling 


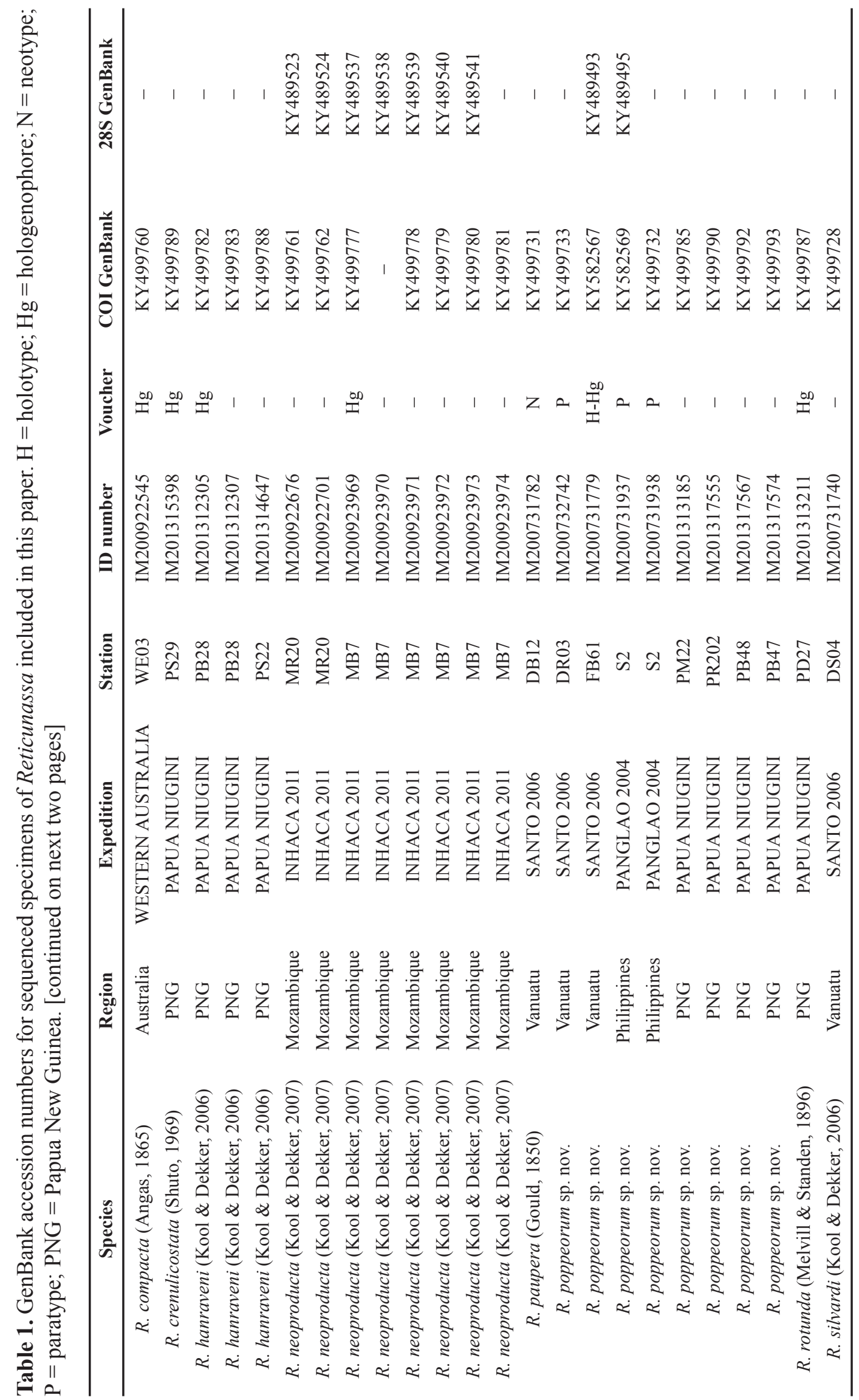




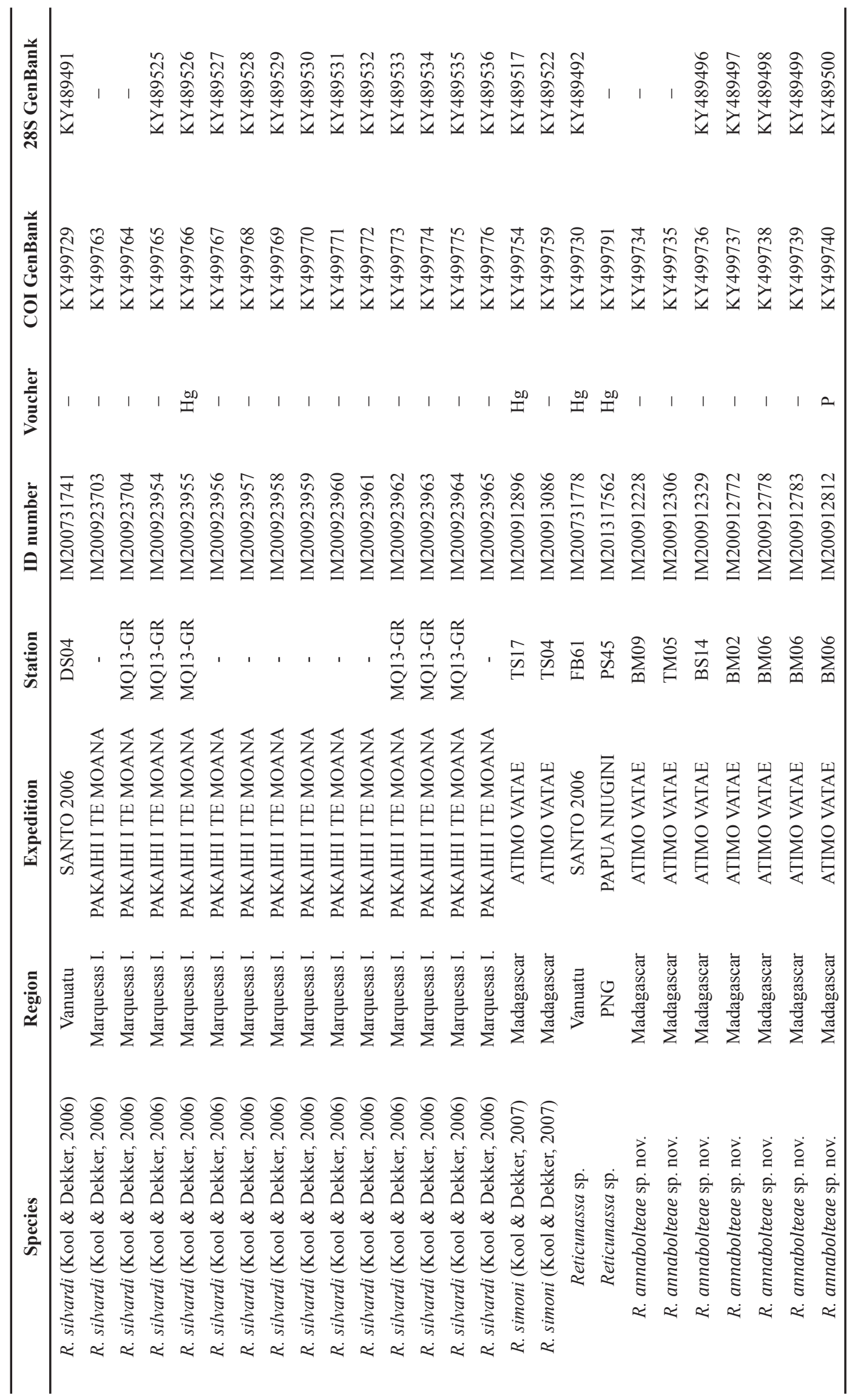




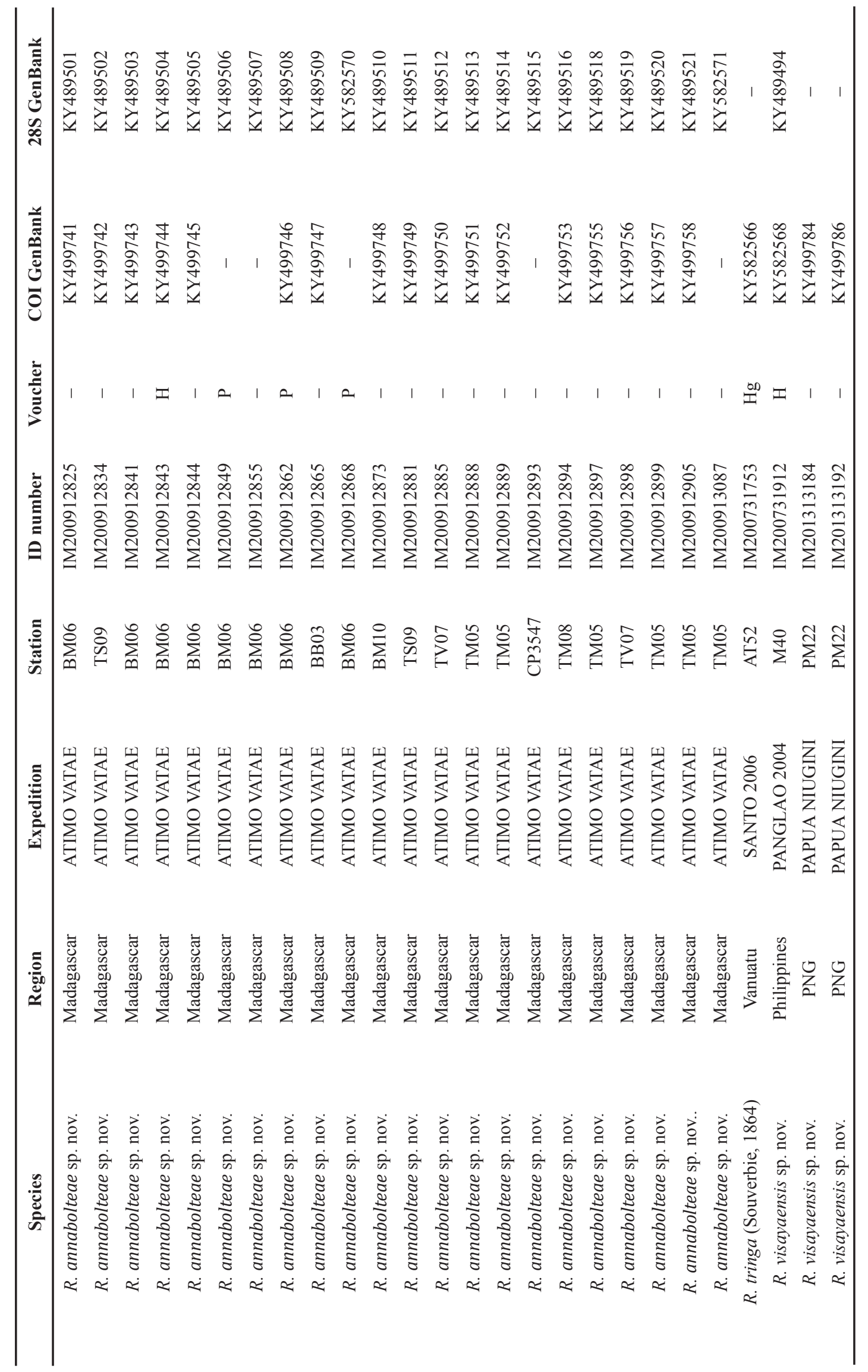


frequency of one tree for every hundred generations) run in eight parallel analyses, was performed using MrBayes v. 3.2.6 (Ronquist \& Huelsenbeck 2003) run on XSEDE. When the log-likelihood scores were found to stabilize, a consensus tree was calculated after omitting the first $25 \%$ trees as burn-in. The analyses were performed using the CIPRES Science Gateway (Miller et al. 2010). Kimura 2-parameter (K2P) distances, estimated with MEGA v. 6 (Tamura et al. 2011), were used to compare each new species with its relatives as defined above.

For type specimen selection, we attributed more importance to the sequenced specimens (genophores sensu Pleijel et al. 2008) than to shell features. All the material (type series and sequences) belongs to MNHN unless otherwise mentioned. Detailed information on the tropical deep sea surveys run by MNHN, from which we obtained most of the new species described herein, can be found in Bouchet et al. (2008) and the BasExp database at http://expeditions.mnhn.fr/.

\section{Results}

A comprehensive dataset on the Nassarius pauperus complex was gathered. For COI, 72 partial sequences were unambiguously aligned without gaps; 244 sites were variable positions, of which 204 were parsimony informative. For $28 \mathrm{~S}, 51$ sequences of the first subunit were successfully amplified. The $28 \mathrm{~S}$ tree supported all the species hypotheses evidenced by COI.

The resulting trees, constructed after the analyses of $C O I$ and $28 \mathrm{~S}$ markers separately (Fig. 1A-B), are in general characterized by strong support values (posterior probability, PP) of deep and terminal nodes. The Nassarius pauperus complex was recovered as a monophyletic group $(\mathrm{PP}=1)$. Branch lengths (sequence divergences), similar to those found for other major clades within Nassariinae (i.e., Phrontis Adams \& Adams, 1853, Nassarius, Tritia Risso, 1826 and Naytia Adams \& Adams, 1853), support our taxonomic decision to consider this clade as a separate genus. Reticunassa is the only available genus group name for this clade, because Nassarius pauperus, its type species, was included in it (see genus remarks for more details). The African clade Naytia is confirmed as the sister group ( $\mathrm{PP}=0.96,0.73)$ of the Indo-Pacific Reticunassa.

Within the Reticunassa clade, two subclades (SC) were recovered: $\mathrm{SC} 1$ is composed of species without spiral sculpture on the intercordal surface (e.g., R. compacta (Angas, 1865), R. rotunda (Melvill \& Standen, 1896), $R$. hanraveni (Kool \& Dekker, 2006) and $R$. silvardi (Kool \& Dekker, 2006)); SC2 includes species with intercordal spiral sculpture (e.g., R. paupera (type species of Reticunassa), $R$. simoni (Kool \& Dekker, 2007), R. crenulicostata (Shuto, 1969), R. tringa (Souverbie, 1864) and R. neoproducta (Kool \& Dekker, 2007)). The COI and 28S taxonomic trees are completely congruent with the morphological hypotheses formulated by Kool \& Dekker $(2006,2007)$. The tree for COI strongly supports the existence of several lineages, which constitute the primary species hypotheses to be delimitated by an integrative approach.

The differences between the intra- and the interspecific genetic distances are shown in Fig. 1C. Among species of Reticunassa, the intraspecific genetic distances range between 0.000 and 0.029 . However, no clear barcode gap (sensu Puillandre et al. 2012b) was found within this Reticunassa data set. Fig. 1C shows two main low frequency regions on the pairwise distance frequencies, one around $2 \%$ and a second near $5 \%$.

ABGD was able to recover 13 groups (Fig. 1): seven correspond to species recognized as valid: G1 $R$. compacta, G2 $R$. rotunda, G3 $R$. hanraveni, G4 $R$. silvardi, G7 R. paupera, G8 $R$. simoni and G9 $R$. crenulicostata. G11 corresponds to a separate lineage represented by a single sequence for a specimen morphologically close to the original description of Nassa tringa Souverbie in Souverbie \& Montrouzier, 1864, a species considered a synonym of Nassarius pauperus by Cernohorsky (1984). 


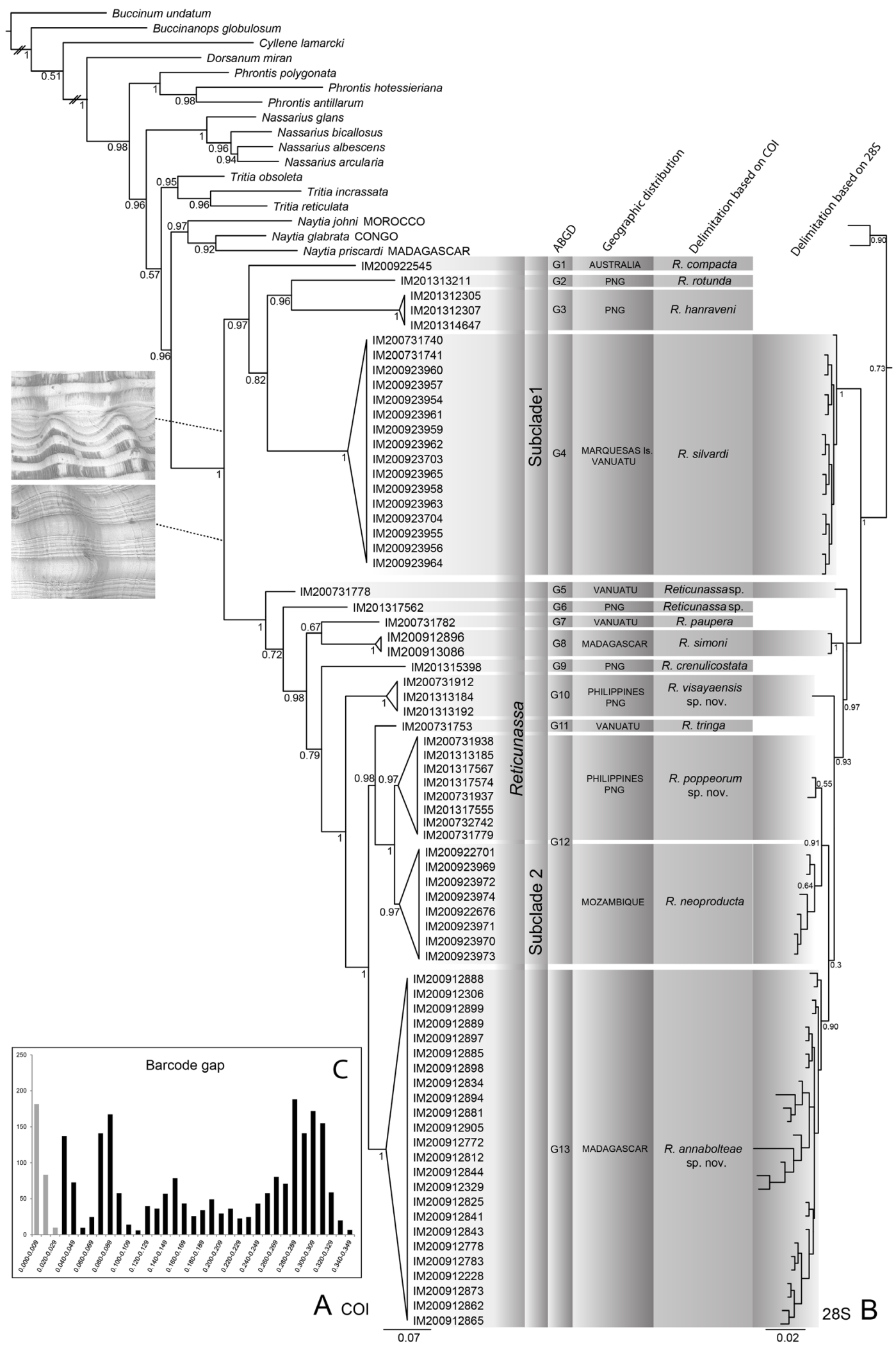

Fig. 1. A. Bayesian tree based on COI sequences of species of Reticunassa. B. Bayesian tree based on $28 \mathrm{~S}$ sequences of species of Reticunassa. C. Frequency of pairwise distance (K2P model) values among COI sequences. In gray, intra-specific variation. In black, inter-specific variation. PNG $=$ Papua New Guinea. 
Four lineages (G5, G6, G10 and G13) potentially correspond to new species. Two of these, G10 and G13, could be distinguished by shell features. However, it was not possible to find differences in shell morphology for the other two species (G5 and G6) and formal descriptions of these await the discovery of informative characters.

The ABGD approach grouped specimens from both Mozambique (originally identified as $R$. neoproducta), the Philippines and Papua New Guinea into G12. Those samples formed two separate (and sister) clades, well supported by reciprocal monophyly in the $C O I(\mathrm{PP}=1)$ and the $28 \mathrm{~S}(\mathrm{PP}=0.91)$ trees, and in accordance with the geographical distribution.

In this paper the genus Reticunassa is redefined, together with its type species. Additionally, six species are described as new to science.

\author{
Class Gastropoda Cuvier, 1797 \\ Subclass Caenogastropoda Cox, 1960 \\ Order Neogastropoda Wenz, 1938 \\ Superfamily Buccinoidea Rafinesque, 1815 \\ Family Nassariidae Iredale, 1916
}

Genus Reticunassa Iredale, 1936

\title{
Type species
}

Nassa paupera Gould, 1850 by original designation (Iredale 1936: 322).

\section{Diagnosis}

Morphological features of the protoconch are of great importance when recognizing species of Reticunassa. Several species have a multispiral protoconch, indicating a planktotrophic/planktonic larval development, resulting in a larger range of distribution. Others have a paucispiral protoconch, indicating a short, non-planktotrophic/direct larval development. All species of Reticunassa possess an unkeeled protoconch (Fig. 2A-B), as do other Nassariinae genera in the Atlantic Ocean (Galindo et al. 2016). However, Reticunassa lives in sympatry with the Indo-Pacific genus Nassarius s. str., characterized by a keeled protoconch. Protoconchs of Reticunassa can be rather broad and flattened; a few are pointed. They might possess microscopic sculpture, such as spiral ridges, axial striae and minute pustules (Fig. 2A-B), but frequently a much higher resolution is needed to see details of this sculpture. Due to the obvious importance of protoconch characters, the identification of specimens in this genus lacking a protoconch becomes more difficult.

Species of Reticunassa have strongly or weakly developed continuous spiral cords, from 10 to 15 on the body whorl (Fig. 2D-F), crossing over the axial ribs. The space between these spiral cords, the intercordal surface, can be smooth, axially striated or can display a number of (occasionally extremely) fine spiral striae (Fig. 2F). This makes the sculpture of the intercordal surface important for identification purposes within Reticunassa. Fig. 1A-B shows that differences on these intercordal surfaces are useful synapomorphies to separate $\mathrm{SC} 1$ from $\mathrm{SC} 2$.

Species within Reticunassa have an axially-ribbed teleoconch (Fig. 2C). These axial ribs may become weaker or absent on the body whorl towards the base and/or the outer lip. Ribs can be round or flat. The number of ribs differs among species. Most species develop a strong varix near the outer lip. The suture is usually deeply impressed. 


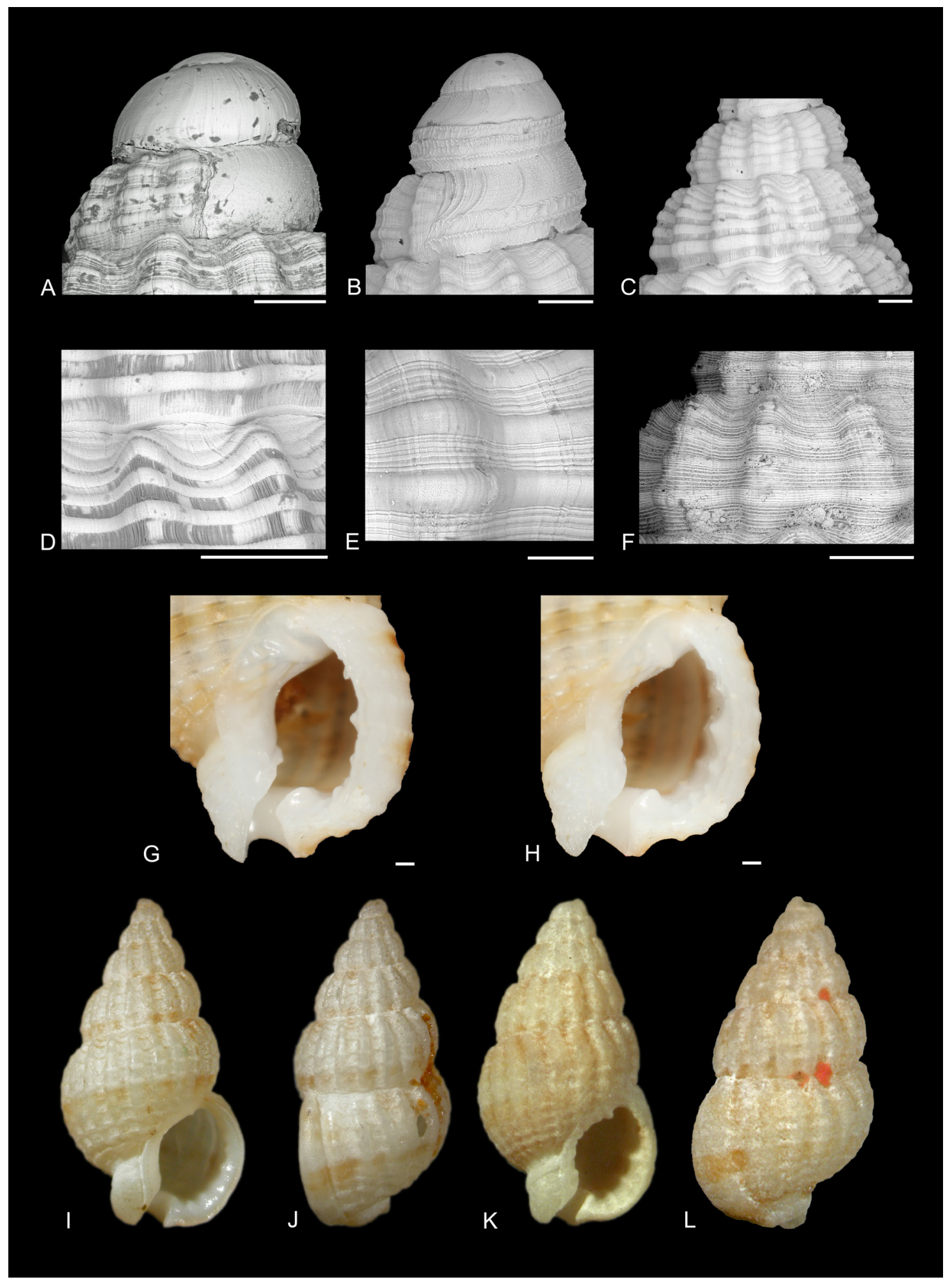

Fig. 2. Characters of Reticunassa Iredale, 1936. A-B. Protoconchs. A. R. paupera (Gould, 1850), MNHN IM-2007-31782. B. R. silvardi (Kool \& Dekker, 2006). C. Suture detail of $R$. paupera. D. Axial striae in R. silvardi. E-F. Intercordal spirals. E. R. tringa (Souverbie, 1864). F. R. paupera. G-H. Columellar details of $R$. paupera. I-L. Type series of $R$. paupera. I-J. Lectotype, USNM 5726, length $9.1 \mathrm{~mm}$, width $4.8 \mathrm{~mm}$. K-L. Paralectotype, MCZ 216602, length $7.2 \mathrm{~mm}$, width $3.5 \mathrm{~mm}$. All scale bars $=200 \mu \mathrm{m}$. 
The aperture is oval to sub-circular, the inside of the outer lip with a number of denticles or lirae (Fig. 2G-H). A columellar callus is present and varies in shape and sculpture. It can be limited to the columella or partly extend over the body whorl.

In some species, the color is variable; generally, it is cream-yellowish to orange, or white to dark brown. Banding with darker colors may also be present. Sometimes only some of the spiral cords are darker than the remaining part of the shell.

Reticunassa species are small, usually varying between 5 and $12 \mathrm{~mm}$, but one is up to $16.5 \mathrm{~mm}$ (R. goliath sp. nov.). Most species live in littoral to sublittoral ecosystems, only empty shells having been found in waters deeper than $260 \mathrm{~m}$. Their occurrence is in the Indo-Pacific Ocean, from the Miocene to the present.

\section{Remarks}

The genus name Reticunassa was introduced to group together "species around pauperus Gould" by Iredale (1936: 322). Cernohorsky (1984: 175) regarded Reticunassa as a synonym of the subgenus "Hima Leach in Gray, 1852", within the genus Nassarius Duméril, 1805. However, Hima is not an available name, but an incorrect subsequent spelling of Hinia Leach in Gray, 1847 (Gray 1847a: 269), since "Hima" included the same three species as in the original description of Hinia (during a period in which original descriptions only consisted of introducing new names). Nonetheless, "Hima" and not Hinia was listed by Adams \& Adams (1853). Cossmann (1901) considered both Hinia and "Hima" as valid names, with "Hima" treated sensu Adams \& Adams (1853), not Leach in Gray, 1852, and with "type species" Nassa incrassata "Müller". Woodring (1928) noted the misspelling and this was reiterated by Lee (2013). Despite this, the error in the use of "Hima" has persisted. On the other hand, Hinia Leach in Gray, 1847 is a junior objective synonym of Tritia Risso, 1826 as they have the same type species, Buccinum reticulatum Linnaeus, 1758, designated for both genera by Gray (1847b: 139). Molecular results of Galindo et al. (2016) indicated that species considered part of the Nassarius pauperus complex, including "Nassarius pauper", form a clade distant from Buccinum reticulatum (accepted as Tritia reticulata), the type species of Tritia (= Hinia), also shown in Fig. 1A. The only available genus group name for this pauperus clade is Reticunassa Iredale, 1936, which is herein reinstated with full generic status.

Other species might be confused as members of the genus Reticunassa because of their morphological similarities. As it is impossible to treat all the small and ridged species, we focus on the following characters: (1) Reticunassa is an Indo-Pacific group of molluscs, not occurring in Atlantic waters (Galindo et al. 2016); (2) species of Reticunassa lack keeled protoconchs (all species with this keel belong to the genus Nassarius); (3) fine spiral striae or axial striae on the intercordal surface (Fig. 2D-F) may be an indication of at least kinship with Reticunassa; (4) all species of Reticunassa have a distinct, elongate and slender shape (Figs 3, 8). These criteria exclude from Reticunassa any Mediterranean species, such as Tritia incrassata (Strøm, 1768) (previously Nassarius incrassatus), West African species, such as Tritia senegalensis (Von Maltzan, 1884) (previously Nassarius senegalensis) and Caribbean species, such as Phrontis candidissima (C.B. Adams, 1845) (previously Nassarius candidissimus) (Galindo et al. 2016). Most Indo-Pacific species, such as Nassarius vanuatuensis Kool \& Galindo, 2014, can be distinguished from Reticunassa by the protoconch features. Additionally, Kool \& Galindo (2014) demonstrated a 19\% genetic distance between Nassarius vanuatuensis and Reticunassa paupera. Naytia priscardi (Bozzetti, 2006) from Madagascar is a member of the sister group of Reticunassa and exhibits great morphological similarity to some species of this genus. Molecular synapomorphies indicate that $N$. priscardi belongs to Naytia. 


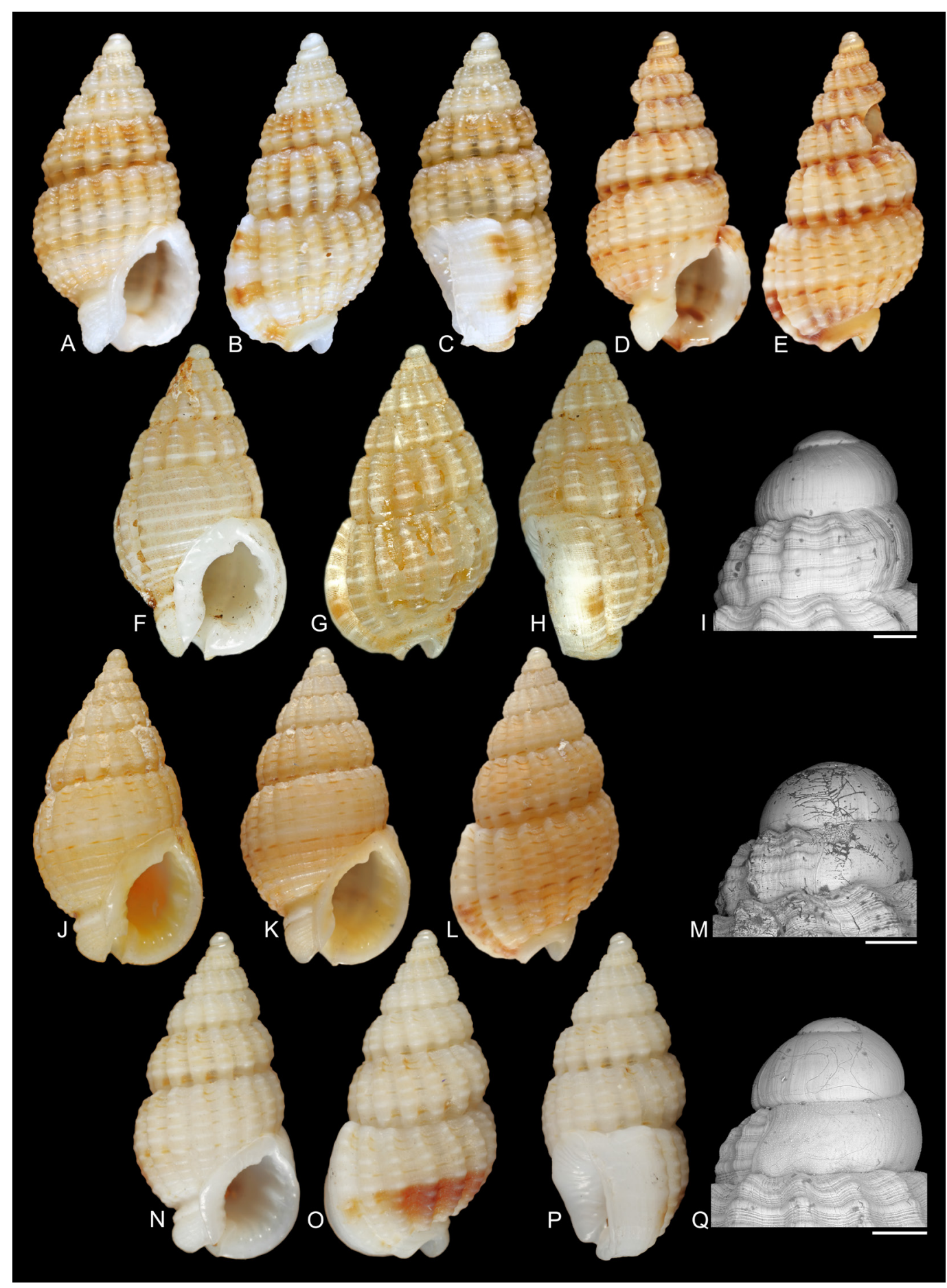

Fig. 3. Shells of Reticunassa Iredale, 1936. - A-C. Possible neotype of R. paupera (Gould, 1850), MNHN IM-2007-31782, length 6.9 mm, width $3.6 \mathrm{~mm}$, Vanuatu, Aore I., $15^{\circ} 36.6^{\prime} \mathrm{S}, 17^{\circ} 10.1^{\prime} \mathrm{E}$, 10-18 m. - D-I. R. tringa (Souverbie, 1864). D-E. Hologenophore, MNHN IM-2007-31753, length $9.5 \mathrm{~mm}$, width $4.6 \mathrm{~mm}$. F-H. Lectotype, MHNBx 2004.TY.89.1, length $9.0 \mathrm{~mm}$, width $5.0 \mathrm{~mm}$. I. Protoconch of the hologenophore. - J-M. $R$. visayaensis sp. nov. J-L. Holotype, MNHN IM2007-31912, length $9.5 \mathrm{~mm}$, width $4.6 \mathrm{~mm}$, Philippines, Panglao I., 9 $9^{\circ} 35.7^{\prime} \mathrm{N}, 123^{\circ} 44.7^{\prime} \mathrm{E}, 0-3 \mathrm{~m}$. M. Protoconch. - N-Q. R. poppeorum sp. nov. N-P. Holotype, MNHN IM-2007-31779, length $9.0 \mathrm{~mm}$, width $4.5 \mathrm{~mm}$, Vanuatu, Aore I., $15^{\circ} 34.4^{\prime} \mathrm{S}, 167^{\circ} 12.6^{\prime} \mathrm{E}, 2-3 \mathrm{~m}$. Q. Protoconch. Scale bars: I, M, Q = $200 \mu \mathrm{m}$. 
Reticunassa paupera (Gould, 1850)

Figs 2A, C, F-L, 3A-C, 4; Tables 1-2

Nassa paupera Gould, 1850: 155.

Nassa paupera - Gould 1852: 12, 262, pl. 19, fig. 330a-b; 1862: 70. — Johnson 1964: 124.

Reticunassa paupera - Iredale 1936: 322.

Nassarius (Hima) pauperus [in part] - Cernohorsky 1972: 186, figs 151-154; 1974: 54, fig. 7; 1984: 176, pl. 37, fig. 1.

Nassarius pauperus - Richard et al. 1984: 12, fig. 24.

\section{Type material}

Lectotype (ICZN Art. 74.5; Johnson 1964: 124)

PACIFIC OCEAN: length $9.1 \mathrm{~mm}$, width $4.8 \mathrm{~mm}$ (USNM 5726, Fig. 2I-J, http://n2t.net/ ark:/65665/3f1 f5ebf5-d0d6-4c9a-bddd-3677d3efb7e2).

Paralectotype (ICZN Art. 74.5; Johnson 1964: 124)

PACIFIC OCEAN: $1 \mathrm{spm}$, type locality, length $7.2 \mathrm{~mm}$, width $3.5 \mathrm{~mm}$ (MCZ 216602, ex USNM, Fig. 2K-L).

\section{Neotype}

Both the lectotype and the paralectotype have been affected by Byne's disease (Kool \& Dekker 2006). However, remnants of microscopic spiral sculpture on the intercordal surface of both specimens are vaguely visible. Gould (1850) did not mention this feature in his original description. The protoconch of the lectotype is badly damaged and that of the paralectotype is completely absent. The protoconch, figured by Cernohorsky (1984: 5, fig. 12) cannot be the protoconch of either of the types, but most likely belongs to Reticunassa tringa, which he considered a synonym of Nassarius pauperus. The poor condition of both existing types calls for the designation of a neotype for this species. Among the specimens in the MNHN collection, there is one sequenced individual that is very similar to the original types of the species. A proposal to designate a neotype is in the process of being submitted to the International Commission on Zoological Nomenclature (ICZN). If accepted, the neotype will be MNHN IM-2007-31782, length $6.9 \mathrm{~mm}$, width $3.6 \mathrm{~mm}$ (Fig. 3A-C; http://coldb.mnhn.fr/catalognumber/mnhn/im/2007-31782).

\section{Type locality}

Pacific Ocean. If the designation of a neotype is accepted by the ICZN, the locality of MNHN IM-200731782 becomes the new type locality: SANTO 2006 Expedition, stn DB12, Vanuatu, South of Aore Island, $15^{\circ} 13.6^{\prime} \mathrm{S}, 167^{\circ} 10.1^{\prime} \mathrm{E}, 10-18 \mathrm{~m}$.

\section{Other material examined}

INDONESIA: Bali, Benoa Port, in sand dumps, leg. T. de Boer (HK 995.06, 1 spm); Bali, Nusa Dua, Benoa Beach, leg. J. Post (HK 995.09, 1 spm); Flores Sea, Pulau Sabalana, Lamuruang, 10-20 m (HD 17295, $1 \mathrm{spm}$ ); Arafura Sea, near Pulau Kuringarankeke, W of Makassar, southern Sulawesi, 5 5'34" S, $119^{\circ} 17^{\prime} 09^{\prime \prime}$ E, leg. H. Morrison (GH, $1 \mathrm{spm}$ ); West-Papua, Manokwari, leg. D. Smits (HK 995.03, 5 spm; HD 38513, 6 spm); SNELLIUS 1929, Kera, near Timor (RMNH, 1 spm); Java, Bay of Jakarta, Pulau Dapur, leg. J.D.F. Hardenberg, 1938 (ZMA.Moll.096216, 1spm); Java, Bay of Jakarta, Pulau Ubi, leg. J.D.F. Hardenberg, 1938 (ZMA.Moll.096218, 4 spm); Java, Bay of Jakarta, Pulau Kelor, leg. J.D.F. Hardenberg, 1938 (ZMA.Moll.096219, 5 spm); Java, Bay of Jakarta, Pulau Untung Djava, leg. J.D.F. Hardenberg, 1938 (ZMA.Moll.096220, 3 spm); Java, Bay of Jakarta, Pulau Dapur, leg. J.D.F. Hardenberg, 1938 (ZMA.Moll.096221, 9 spm); Java, Bay of Jakarta, Pulau Damar, leg. J.D.F. Hardenberg, 1938 (ZMA. Moll.096222, 2 spm); Java, Bay of Jakarta, Pulau Sakit, leg. J.D.F. Hardenberg, 1938 (ZMA.Moll.096223, 


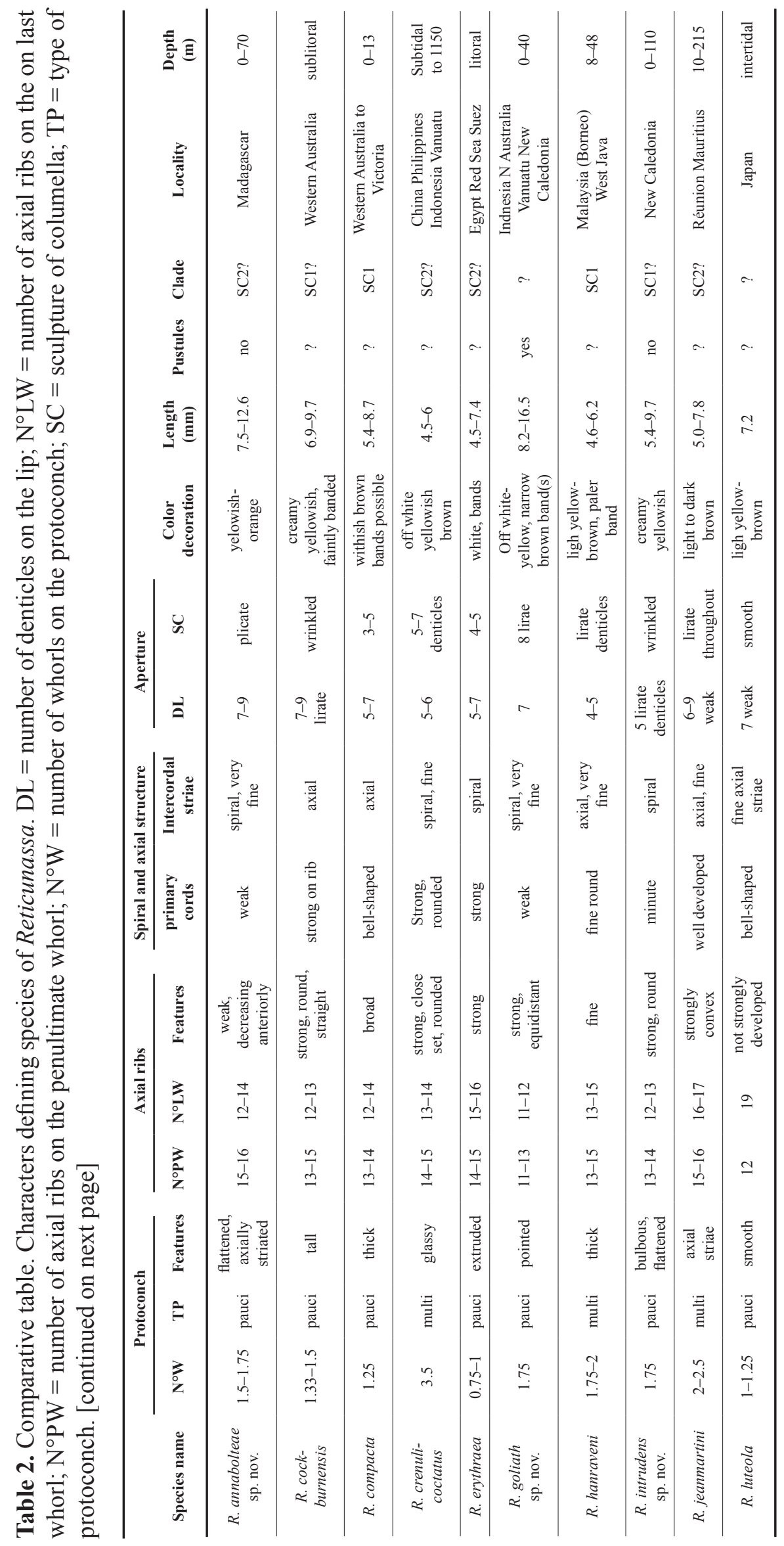




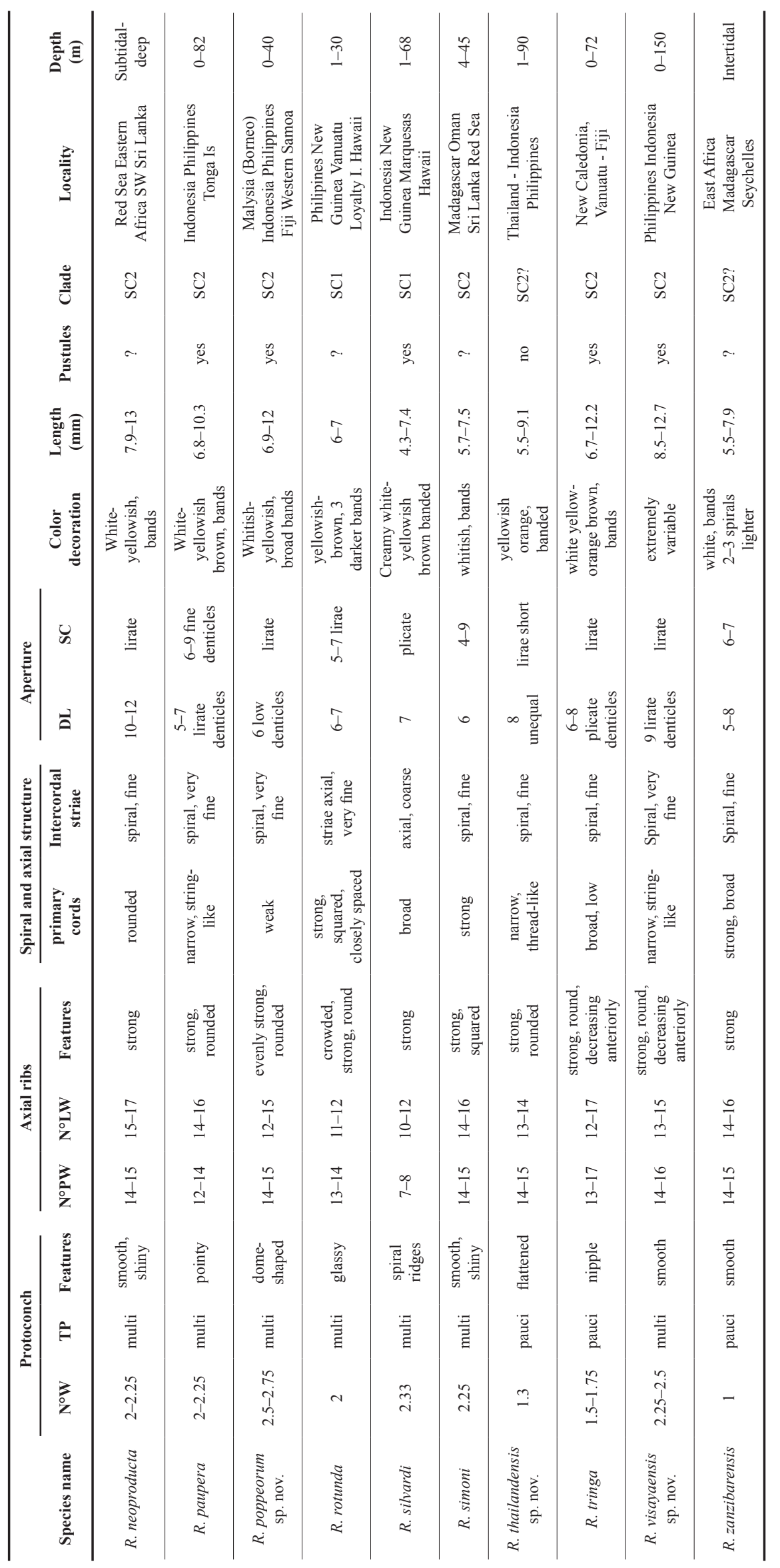


3 spm); Java, Bay of Jakarta, Pulau Njamoek, leg. J.D.F. Hardenberg, 1938 (ZMA.Moll.096224, 10 spm); Java, Bay of Jakarta, Pulau Noesi, leg. J.D.F. Hardenberg, 1938 (ZMA.Moll.096225, 15 spm); Java, Bay of Jakarta, Pulau Ubi, leg. J.D.F. Hardenberg, 1938 (ZMA.Moll.096226, 9 spm); Java, Bay of Jakarta, Pulau Ramboet, leg. J.D.F. Hardenberg, 1938 (ZMA.Moll.096227, 1 spm); KEPULAUAN SERIBU 2005, Java, Hantu Besar, 5³1.8' S, 106³2.3' E, 5-26 m (ZMA.Moll.175389, 1 spm); KEPULAUAN SERIBU 2005, stn SER05/33, Java, Pari East, 551.3’ S, 106³8.3’ E, 5-25 m (ZMA, 1 spm); KEPULAUAN SERIBU 2005, stn SER05/43, Java, Payung Besar, 540.3' S, 106³3.8' E, 5-25 m (ZMA.Moll.154531, $2 \mathrm{spm}$ ).

PHILIPPINES: all PANGLAO 2004: stn M10, Panglao I., Bingag/Tabalong, 9³7.8' N, $123^{\circ} 48.4^{\prime}$ E, 0-3 m (1 spm); stn M26, Bohol I., Taloto, 940.9' N, 12351.1' E, 0-2 m (1 spm); stn S25, Bohol I.,

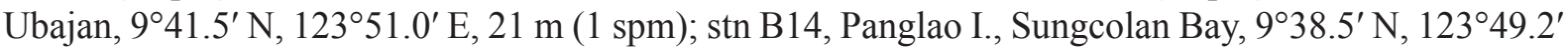
E, $2-4 \mathrm{~m}(3 \mathrm{spm})$.

AUSTRALIA: Queensland, Fitzroy I. (HK 995.08, 2 spm; HD 17298, 1 spm; PS, 1 spm); Queensland, Innisfail, leg. Pini (ZMA.Moll.096207, 1 spm).

GUAM: Neye I. Channel, leg. S.P. Kool (HK 995.04, 2 spm); Apra Harbor, by diving to $10 \mathrm{~m}$, leg. H. Morrison (HK 995.05, 1 spm); Glass Breakwall, snorkeling, 1-2 m (HD 12297, 2 spm); Glass Breakwall, $6 \mathrm{~m}$, in rubble (GH, $1 \mathrm{spm})$.

CAROLINE ISLANDS: Truk I., Moen I. (HK 995.10, 2 spm).

NEW CALEDONIA: CHALCAL 1: stn D17, Plateau Chesterfield-Bellona, $19^{\circ} 11^{\prime} \mathrm{S}, 158^{\circ} 55.8^{\prime} \mathrm{E}, 44 \mathrm{~m}$

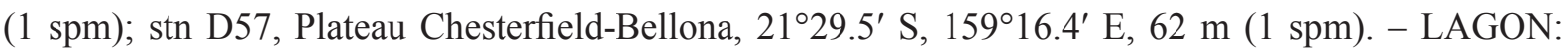
Ile Quen, Baie Prony, stn 64, 22 $28^{\prime}$ S, 166 $25^{\prime}$ E, 15 m (2 spm; NBC, 1 spm; HK 995.01, 1 spm); Ile

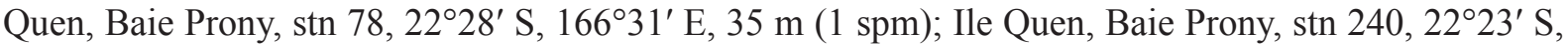

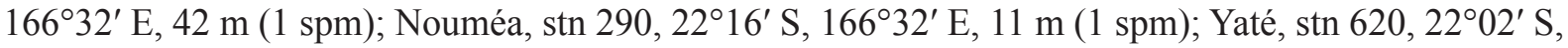

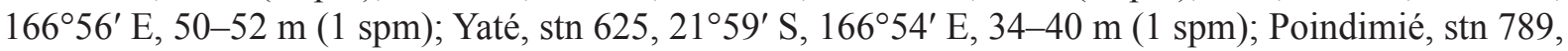
$21^{\circ} 03^{\prime} \mathrm{S}, 164^{\circ} 34^{\prime} \mathrm{E}, 29 \mathrm{~m}$ (1 spm); Poum, stn 1039, Balabio Section, $20^{\circ} 01^{\prime} \mathrm{S}, 164^{\circ} 02^{\prime} \mathrm{E}, 20-21 \mathrm{~m}(1$

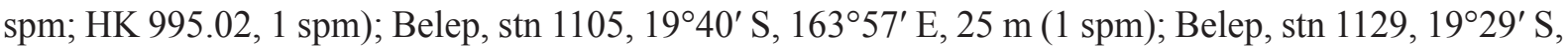
$163^{\circ} 49^{\prime}$ E, $40 \mathrm{~m}(1 \mathrm{spm})$; Belep, stn 1168, 19 ${ }^{\circ} 16^{\prime} \mathrm{S}, 163^{\circ} 09^{\prime} \mathrm{E}, 50 \mathrm{~m}$ (2 spm); Nouméa, Pointe Magnin, stn $1355,22^{\circ} 18.9^{\prime} \mathrm{S}, 166^{\circ} 26.6^{\prime} \mathrm{E}, 7-10 \mathrm{~m}$ (3 spm). - MONTROUZIER 1993: Touho, Lagon du grand Récif Mengalia, stn 1253, 20 46.6' S, $165^{\circ} 14.1^{\prime}$ E, 2-3 m (1 spm); Touho, Lagon du grand Récif Mengalia, stn 1266, 20³9.6' S, $165^{\circ} 14.7^{\prime} \mathrm{E}, 10-15 \mathrm{~m}$ (1 spm); Touho, Lagon du grand Récif Mengalia, stn 1269, $20^{\circ} 35.1^{\prime} \mathrm{S}, 165^{\circ} 08.1^{\prime} \mathrm{E}, 15-20 \mathrm{~m}$ (2 spm); Koumac, stn 1298, 2035.2' S, $1^{\circ} 64^{\circ} 16.6^{\prime} \mathrm{E}, 2-4 \mathrm{~m}$ (1 spm); Koumac, stn 1299, 20³4' S, $164^{\circ} 13.0^{\prime}$ E, $12-14$ m (11 spm; NBC, 1 spm; HD 24147, 1 spm); Koumac, stn $1308,24^{\circ} 40^{\prime} \mathrm{S}, 164^{\circ} 15.2^{\prime} \mathrm{E}, 15-20 \mathrm{~m}$ (3 spm; ZMA, $1 \mathrm{spm}$ ); Koumac, stn 1312, 20 $40^{\prime} \mathrm{S}, 164^{\circ} 14.9^{\prime} \mathrm{E}$, 26-40 m (6 spm; HK 995.11, 1 spm); Koumac, stn 1314, 20³9.8' S, 164¹5.3' E, 30-63 m (7 spm); Koumac, stn $1315,20^{\circ} 40.7^{\prime} \mathrm{S}, 164^{\circ} 14.7^{\prime} \mathrm{E}, 66-87 \mathrm{~m}$ (3 spm); Koumac, stn 1318, $20^{\circ} 41.4^{\prime} \mathrm{S}, 164^{\circ} 14.8^{\prime} \mathrm{E}$,

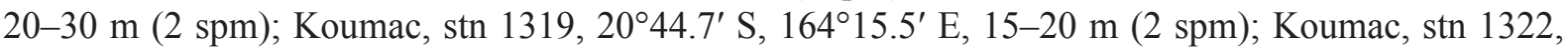
$20^{\circ} 45.2^{\prime} \mathrm{S}, 1^{\circ} 64^{\circ} 15.2^{\prime} \mathrm{E}, 53-71 \mathrm{~m}(1 \mathrm{spm})$; Koumac, stn $1323,20^{\circ} 40.9^{\prime} \mathrm{S}, 1^{\circ} 4^{\circ} 14.8^{\prime} \mathrm{E}, 82-120 \mathrm{~m}$ (1 spm). - CORAIL 2: stn DW32, Plateau de Chesterfield, $19^{\circ} 25^{\prime} \mathrm{S}, 158^{\circ} 49^{\prime} \mathrm{E}, 55 \mathrm{~m}(1 \mathrm{spm})$; stn DW34, Plateau de Chesterfield, $19^{\circ} 18^{\prime} \mathrm{S}, 158^{\circ} 47^{\prime}$ E, $47 \mathrm{~m}$ (2 spm); stn DW 38, Plateau de Chesterfield, $19^{\circ} 22^{\prime} \mathrm{S}, 158^{\circ} 43^{\prime}$ E, $61 \mathrm{~m}(1 \mathrm{spm})$; stn DW56, Plateau de Chesterfield, $19^{\circ} 18^{\prime} \mathrm{S}, 158^{\circ} 47^{\prime} \mathrm{E}, 66 \mathrm{~m}(1$ spm); stn DW59, Plateau de Chesterfield, $19^{\circ} 19^{\prime} \mathrm{S}, 158^{\circ} 57^{\prime} \mathrm{E}, 50 \mathrm{~m}$ (1 spm); stn DW65, Plateau de Chesterfield, $19^{\circ} 15^{\prime} \mathrm{S}, 158^{\circ} 41^{\prime} \mathrm{E}, 62 \mathrm{~m}$ (1 spm); stn DW71, Plateau de Chesterfield, $19^{\circ} 15^{\prime} \mathrm{S}, 158^{\circ} 24^{\prime} \mathrm{E}$, $55 \mathrm{~m}$ (HD 22467, $1 \mathrm{spm})$; stn DW72, Plateau de Chesterfield, $19^{\circ} 15^{\prime} \mathrm{S}, 158^{\circ} 21^{\prime} \mathrm{E}, 32 \mathrm{~m}$ (1 spm);

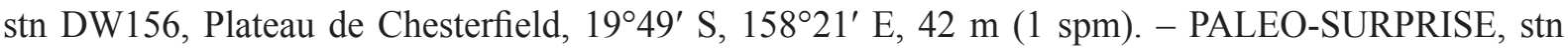
DW1382, $18^{\circ} 26.5^{\prime} \mathrm{S}, 163^{\circ} 05.9^{\prime} \mathrm{E}, 41-42 \mathrm{~m}$ (1 spm). 
LOYALTY ISLANDS: both LIFOU 2000: Lifou, Santal Bay, stn 1423, 2054.0' S, $167^{\circ} 07.1^{\prime} \mathrm{E}, 12 \mathrm{~m}$ (2 spm); Lifou, Santal Bay, stn 1444, 2055.0' S, 16705.2' E, 9-20 m (1 spm).

VANUATU: all SANTO 2006: stn FB56, NE of Tangisi I., $15^{\circ} 35.2^{\prime} \mathrm{S}, 167^{\circ} 02.1^{\prime} \mathrm{E}, 3-18 \mathrm{~m}$ (3 spm);

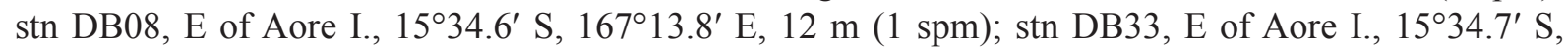
$167^{\circ} 13.8^{\prime} \mathrm{E}, 14-25 \mathrm{~m}(1 \mathrm{spm})$; stn ZB36, E of Aore I., $15^{\circ} 34.3^{\prime} \mathrm{S}, 167^{\circ} 12.4^{\prime} \mathrm{E}$, intertidal (1 spm);

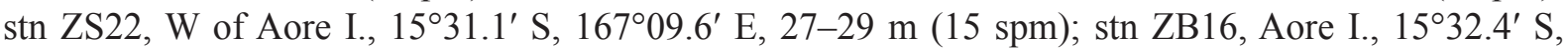

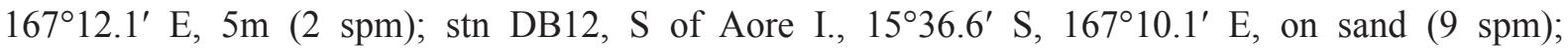
stn LD35, Aore I., $15^{\circ} 32.7 / 32.8^{\prime} \mathrm{S}, 167^{\circ} 11.5 / 11.6^{\prime} \mathrm{E}, 3-8 \mathrm{~m}$ (1 spm); stn DB48, W of Malo I., 16 $38.7^{\prime} \mathrm{S}$, $167^{\circ} 5.2^{\prime} \mathrm{E}, 10-17 \mathrm{~m}(1 \mathrm{spm})$; stn DB83, E of Malo I., $15^{\circ} 43.4^{\prime} \mathrm{S}, 167^{\circ} 15.0^{\prime} \mathrm{E}$, on sand $(2 \mathrm{spm})$; stn DS49, Malo I., $15^{\circ} 38.7^{\prime} \mathrm{S}, 167^{\circ} 05.2^{\prime} \mathrm{E}, 10-17 \mathrm{~m}$ (4 spm); stn ZM15, NW of Malo I., $15^{\circ} 38.1^{\prime} \mathrm{S}$, $167^{\circ} 05.9^{\prime} \mathrm{E}$, intertidal (1 spm); stn DB75, SE of Matewulu, $15^{\circ} 22.9^{\prime} \mathrm{S}, 167^{\circ} 11.9^{\prime} \mathrm{E}, 20 \mathrm{~m}$ (4 spm);

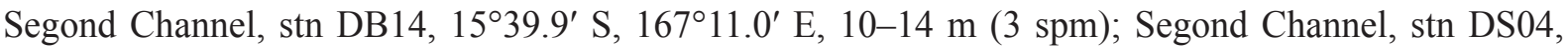
$15^{\circ} 31.4^{\prime} \mathrm{S}, 167^{\circ} 14.1^{\prime}$ E, 25 m (33 spm; HD 38512, 2 spm; HK 995.12, 3 spm); Segond Channel, stn

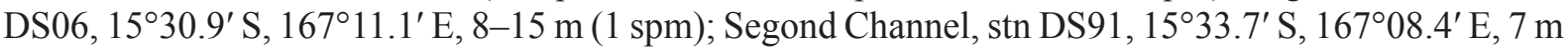

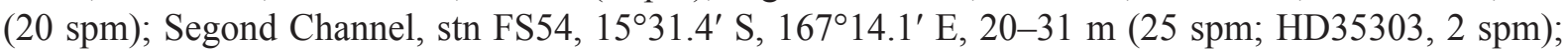
Segond Channel, stn FS77, 15³3.1' S, 16709.6' E, 29 m (17 spm; HK 995.14, 2 spm); Segond Channel,

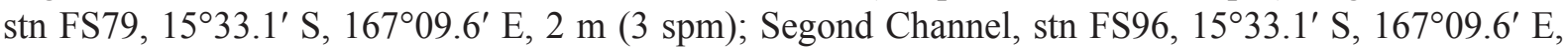

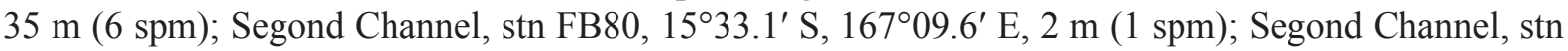

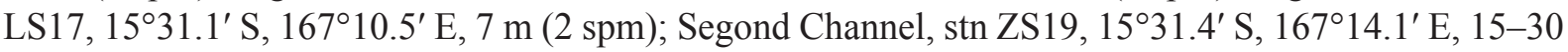
m (20 spm); Segond Channel, stn NB12, 15³3.1' S, 167009.6' E, 20 m (1 spm); Segond Channel, stn NS37, $15^{\circ} 31.4^{\prime} \mathrm{S}, 167^{\circ} 09.8^{\prime}$ E, 2-3 m (4 spm); Palikulo Bay, stn DB53, $15^{\circ} 28.8^{\prime} \mathrm{S}, 167^{\circ} 15.2^{\prime} \mathrm{E}, 5 \mathrm{~m}(2$

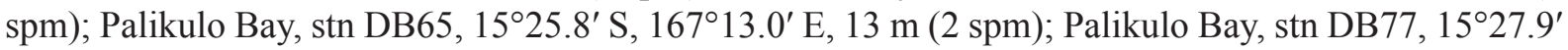

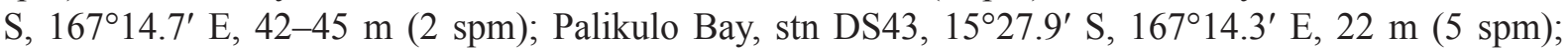

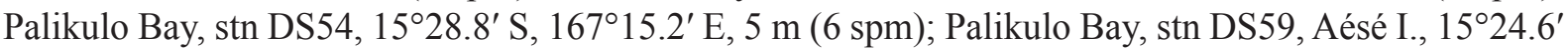
S, $167^{\circ} 14.3^{\prime}$ E, 6-43 m (1 spm); Palikulo Bay, stn DB16, Tutuba I., $15^{\circ} 35.5^{\prime} \mathrm{S}, 167^{\circ} 15.8^{\prime} \mathrm{E}, 32-40 \mathrm{~m}$

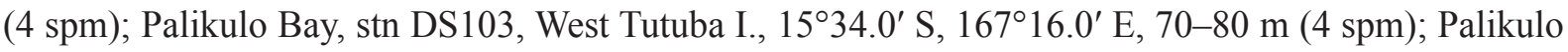
Bay, stn LD36, West Taluba I.,15³9.5' S, 167¹6.8' E, 8-10 m (1 spm); Palikulo Bay, stn DS105, NW

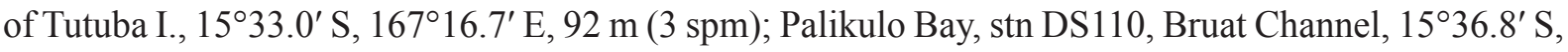
$167^{\circ} 08.8^{\prime}$ E, $35 \mathrm{~m}$ (1 spm); Palikulo Bay, stn LD12, Bruat Channel, $15^{\circ} 36.6^{\prime} \mathrm{S}, 167^{\circ} 11.3^{\prime} \mathrm{E}, 2-4 \mathrm{~m}$ (1 spm); Palikulo Bay, stn ZB06, SW Urélapa I., 15³6.8' S, 16701.3' E (1 spm); SE corner of SANTO 2006, shallow water (10 spm; NBC, 1 spm; HD 38511, 1 spm; HK 995.13, 2 spm).

FIJI: MUSORSTOM 10, stn DW1384, 18²18.5' S, 17806' E, 260-305 m (1 spm).

TONGA: BORDAU 2, stn DW1581, $18^{\circ} 41^{\prime} \mathrm{S}, 174^{\circ} 02^{\prime} \mathrm{E}, 75-85 \mathrm{~m}(1 \mathrm{spm})$.

\section{Description}

Protoconch. Approximately 2.25 shiny whorls (Fig. 2A). SEM photography shows fine axial striae and minute pustules on last whorl.

SHELL. Teleoconch consists of approximately 5 convex whorls. Suture deep. Penultimate whorl with 12-14 and body whorl with 14-16 pronounced and rounded axial ribs. Outer lip with a strong varix.

SPIRAL CORDS. Penultimate whorl with 5-6 and body whorl with 10-11 continuous, narrow, string-like spiral cords, some more pronounced on axial ribs than in between them.

InTERCORDAL SCULPTURE. Between spiral cords, spiral striae faintly visible on well-preserved specimens (Fig. 2F). 
APERTURE. Aperture oval-rounded, outer lip with 5-7 fairly prominent, lirate denticles, peripheral denticle most prominent. Columella (Fig. 2G-H) with 6-9 fine denticles, columellar callus sharply bordered and limited to columella. Parietal denticle strong, anal canal wide.

OPERCULUM. Whitish, serrated.

SiPHONAL CANaL. Fasciole strong, siphonal area with 5-7 weak cords, last one most prominent.

CoLor. Creamy-white to yellowish brown, occasionally with 1-3 darker spiral bands.

Adult SIZE. $6.8-10.3 \mathrm{~mm}$, usually $7.5-8.5 \mathrm{~mm}$.

\section{Remarks}

Several species, previously synonymized with $R$. paupera by Cernohorsky (1984), have been taken out of synonymy in the past few years. A series of published reviews (Kool \& Dekker 2006, 2007), including the present article, demonstrate that the Nassarius pauperus complex is composed of several valid species. Therefore, the synonymy given for Reticunassa paupera s. str. has become very short and caution is advised with citations of this species name in the literature.

The combination of the multispiral protoconch, the many pronounced, round ribs, the deep suture, the broad varix, the very fine spirals, the sharply-bordered columella and the columellar denticles not lirae characterize this species, one of the smaller within the genus.

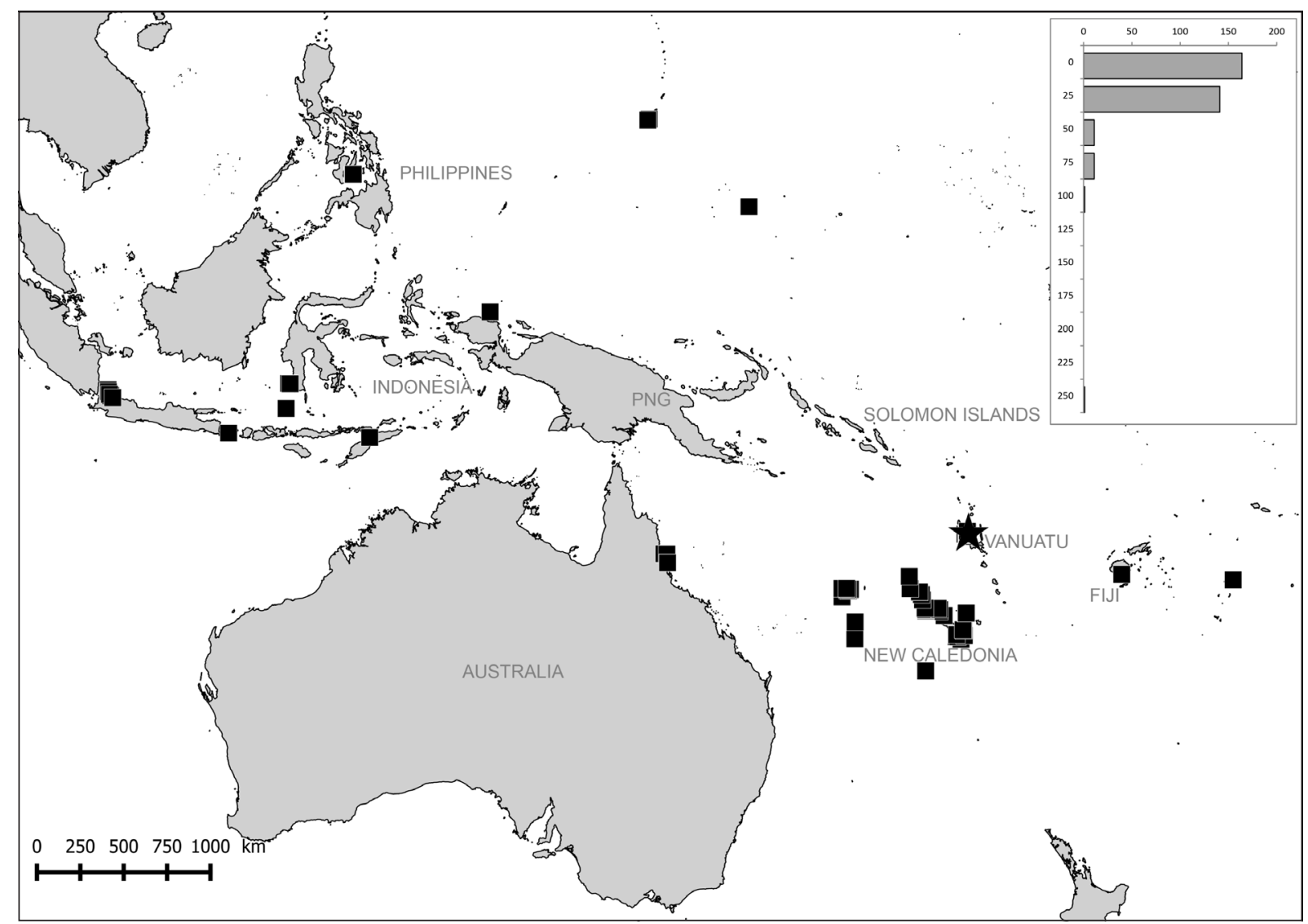

Fig. 4. Geographical and bathymetrical distribution of Reticunassa paupera (Gould, 1850). Star indicates type locality. Each bar represents all lv or dd specimens. 
The differences between $R$. paupera and the other species of Reticunassa, described below, will be discussed in the remarks section in each case.

In the taxonomic history of Nassa paupera, several declensions on the specific epithet have been used. In Latin, there are two different adjectives with the same meaning: the classical pauper $\left(3^{\text {rd }}\right.$ declension, nominative unchanged) and the post-classical (medieval, generally clerical) pauperus ( $1^{\text {st }}$ declension, paupera feminine, pauperum neuter), both meaning "poor." The second adjective is obviously a derivation of the first. The fact that Gould (1850) used paupera in his original description indicates that he was referring to the second meaning. Combined with Reticunassa, the new combination is R. paupera (Gould, 1850).

\section{Habitat}

Specimens were collected from the intertidal to a depth of $82 \mathrm{~m}$, mostly from 0 to $25 \mathrm{~m}$; one empty shell was obtained at $260 \mathrm{~m}$ (Fiji Islands). The proposed neotype was collected on sand with dead corals. Specimens from Guam (HD 12797) were found in rubble.

\section{Distribution}

Widely distributed in the western Pacific Ocean, from Indonesia and the Philippines to the Tonga Islands (Fig. 4).

Reticunassa tringa (Souverbie, 1864)

Figs 2E, 3D-I, 5; Tables 1-2

Nassa tringa Souverbie in Souverbie \& Montrouzier, 1864: 272, pl. 10, fig. 7.

Nassa mamillata Preston, 1907: 267, textfig. 1.

Nassarius (Hima) pauperus [in part] - Cernohorsky 1972: 186, fig. 152 only; 1984: 176, pl. 37, figs 5-6 (syntypes of Nassa tringa), fig. 7 (holotype of N. mamillata).

\section{Type material}

NEW CALEDONIA: 6 syntypes of Nassa tringa (MHNBx 2014.TY.89.0, 2 spm; MNHN IM-200028389, $4 \mathrm{spm}$, http://coldb.mnhn.fr/catalognumber/mnhn/im/2000-28389). We designate MHNBx 2004.TY.89.1 (from the former MHNBx 2004.TY.89.0) as the lectotype (Fig. 3F-H), length $9.0 \mathrm{~mm}$, width $5.0 \mathrm{~mm}$.

NEW CALEDONIA: holotype of N. mamillata (NHMUK 1923.2.20.8).

\section{Other material examined}

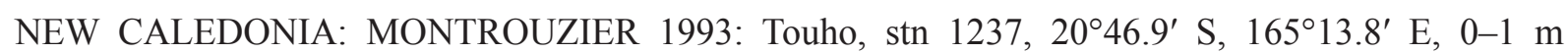
(15 spm; HD, 2 spm; HK 994.02, 3 spm); Touho, stn 1240, 2046.5' S, 165¹4.0' E, 0-2 m (19 spm;

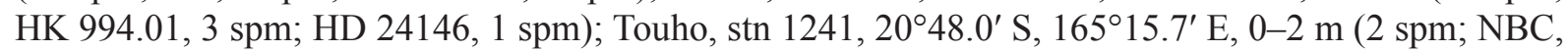
$1 \mathrm{spm})$; Touho, stn $1242,20^{\circ} 46.2^{\prime} \mathrm{S}, 165^{\circ} 14.5^{\prime} \mathrm{E}(29 \mathrm{spm})$; Touho, stn $1244,20^{\circ} 49.3^{\prime} \mathrm{S}, 165^{\circ} 15.2^{\prime} \mathrm{E}$ (1 spm); Touho, stn $1245,20^{\circ} 45.2^{\prime} \mathrm{S}, 165^{\circ} 16.3^{\prime} \mathrm{E}$ (3 spm); Touho, stn $1246,20^{\circ} 42.8^{\prime} \mathrm{S}, 1^{\circ} 05^{\circ} 08.7^{\prime} \mathrm{E}$

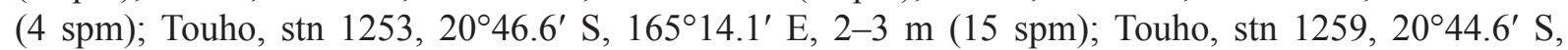
$165^{\circ} 13.7^{\prime} \mathrm{E}, 15-35 \mathrm{~m}$ (2 spm); Touho, stn $1261,20^{\circ} 46^{\prime}-20^{\circ} 47^{\prime} \mathrm{S}, 165^{\circ} 15^{\prime}-165^{\circ} 16.5^{\prime} \mathrm{E}, 45-56 \mathrm{~m}$ (2 spm); Koumac, stn 1277, $20^{\circ} 34^{\prime} \mathrm{S}, 164^{\circ} 16^{\prime} \mathrm{E}, 0-2 \mathrm{~m}$ (4 spm); Koumac, stn $1278,20^{\circ} 34^{\prime} \mathrm{S}, 1^{\circ} 4^{\circ} 16^{\prime} \mathrm{E}$, 0-2 m (2 spm); Koumac, stn 1279, 20 $0^{\circ} 35^{\prime} \mathrm{S}, 164^{\circ} 15.5^{\prime} \mathrm{E}$ (3 spm); Koumac, stn 1282, $20^{\circ} 33.5^{\prime} \mathrm{S}$,

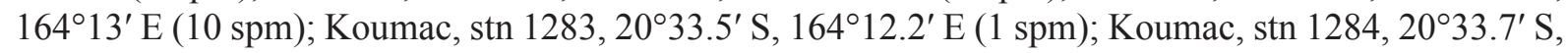
$164^{\circ} 19^{\prime}$ E (19 spm); Koumac, stn 1286, 20 $38^{\prime}-20^{\circ} 39^{\prime} \mathrm{S}, 164^{\circ} 16^{\prime}-164^{\circ} 17^{\prime}$ E (2 spm); Koumac, stn $1288,20^{\circ} 40^{\prime} \mathrm{S}, 1^{\circ} 4^{\circ} 19^{\prime} \mathrm{E}(1 \mathrm{spm})$; Koumac, stn $1289,20^{\circ} 29.2^{\prime} \mathrm{S}, 1^{\circ} 4^{\circ} 10.2^{\prime} \mathrm{E}$ (6 spm); Koumac, stn $1290,20^{\circ} 29.2^{\prime} \mathrm{S}, 164^{\circ} 10.2^{\prime} \mathrm{E}$ (1 spm); Koumac, stn $1291,20^{\circ} 22.4^{\prime} \mathrm{S}, 164^{\circ} 06.8^{\prime} \mathrm{E}$ (1 spm); Koumac, 


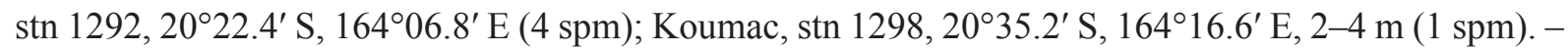

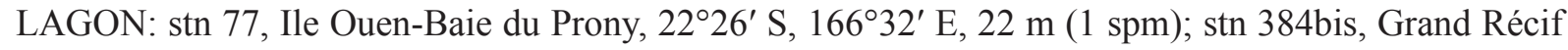
Sud, $22^{\circ} 34^{\prime} \mathrm{S}, 167^{\circ} 11^{\prime} \mathrm{E}, 72 \mathrm{~m}$ (1 spm); stn 441, Atoll de Huon, $18^{\circ} 04^{\prime} \mathrm{S}, 162^{\circ} 56^{\prime} \mathrm{E}, 37 \mathrm{~m}$ (1 spm). New Caledonia, leg. Rossiter, ex coll. Dautzenberg (ZMA.Moll.098635, $5 \mathrm{spm}$ ); beach S of Noumea, leg. Mrs Wijsman-Best, 1968 (ZMA Moll. 096210, 2 spm); Ile des Pins (PS, 2 spm); Nouméa, leg. A.P. Greeven-Lels, 1965 (HD 21379, 9 spm).

VANUATU: SANTO 2006: stn AT52, Segond Channel, 15³1.5' S, 167¹2.7' E, 52-62 m (MNHN IM2007-31753, 1 lv, 19.5 mm, w 4.6 mm, Fig. 3D-E, I); stn LM23, Segond Channel, 15³1.5' S, $167^{\circ} 09.6^{\prime}$ E,

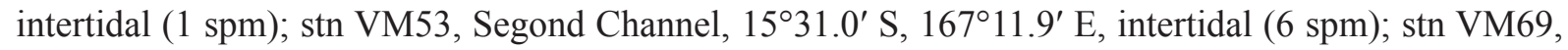
Tutuba I., $15^{\circ} 33.4^{\prime} \mathrm{S}, 167^{\circ} 16.7^{\prime} \mathrm{E}$, intertidal (MNHN IM-2007-31758, $1 \mathrm{lv}$ ); stn FB92, Tutuba I.,

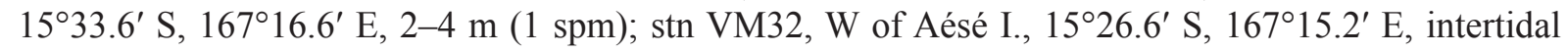
(2 spm). - Efate, snorkeling, 2-3 m, leg. T. McCleery, 2002 (HD 17091, 4 spm; HK 994.20, 3 spm).

FIJI: Yasawa I., leg. S.P. Kool (coll. S.P. Kool, 3 spm; HK 994.26, 1 spm).

\section{Description}

Protoconch. Whitish, paucispiral, consisting of 1.5-1.75 whorls (Fig. 3I). SEM photograph shows very fine growth lines, and in addition minute pustules on part of last whorl.

SHELL. Elongate-ovate, teleococh with 5-6 whorls, suture impressed. On penultimate whorl 13-17 and on body whorl 12-17 pronounced, round axial ribs, on ventral side of body whorl often decreasing in height towards base.

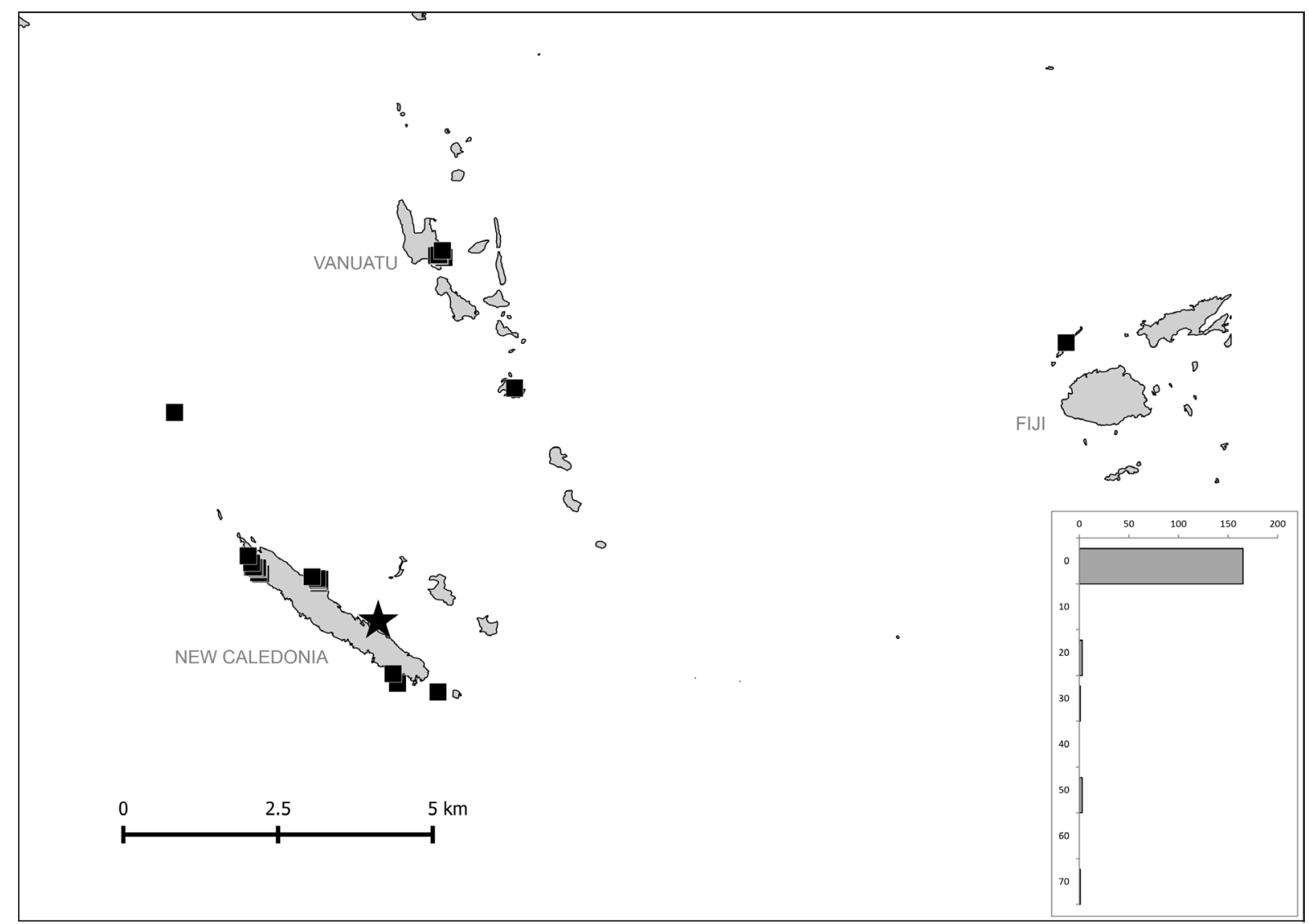

Fig. 5. Geographical and bathymetrical distribution of Reticunassa tringa (Souverbie, 1864). Star indicates type locality. Each bar represents all lv or dd specimens. 
SPIRAL CORDS. Continuous, moderately broad, rounded primary cords, approximately $4-5$ on penultimate and approximately 10-12 on body whorl.

INTERCORDAL SCULPTURE. Fine, evenly-spaced, continuous striae (Fig. 2E), 7-9 on body whorl, sometimes visible between, as well as on spiral cords.

APERTURE. Roundish; outer lip thick, plicate inside, with strong varix; peripheral denticle and more pronounced pleat near siphonal canal. Columella callused, callus sharply bordered, posteriorly slightly extending over body whorl. Columellar lip lirate throughout. Parietal denticle moderately strong.

Operculum. Yellowish, serrated.

Siphonal canal. Narrow, with strong fasciole. Siphonal area with weak cords and in some specimens faint axial striae between cords.

Color. Off white, yellowish to orange or brown. Banding very variable: some specimens with chestnut to dark brown, or nearly black bands; on penultimate whorl a sub- and/or a supra-sutural band; on body whorl occasionally one band directly below periphery and one at base. Occasionally, some or most spiral cords darker, especially between axial ribs. Aperture yellowish, showing outside banding, if present.

Adult SIZE. 6.7-12.2 mm, usually $8-9.5 \mathrm{~mm}$.

\section{Remarks}

Characteristics of this species are a paucispiral protoconch, low spiral cords, in combination with numerous, evenly-spaced intercordal spiral striae (Fig. 2E), axial ribs decreasing in height anteriorly on the ventral side of the body whorl, and the presence of lirae on the columellar callus. In wellpreserved specimens, spiral striae are also visible on top of the spiral cords. The intercordal spiral striae were not mentioned in the original description by Souverbie (in Souverbie \& Montrouzier, 1864). However, Preston (1907) mentioned this sculpture in the description of Nassa mamillata. We agree with Cernohorsky (1984), that the latter is a synonym of Reticunassa tringa.

$R$. tring a can easily be distinguished from $R$. paupera by its paucispiral protoconch, probably figured by Cernohorsky (1984: 5, fig. 12). Cernohorsky did not discuss protoconch morphology or other conchological features shared by species within the Nassarius pauperus complex, resulting in numerous synonyms of $R$. paupera, followed by many authors.

$R$. tringa closely resembles $R$. visayaensis sp. nov. and $R$. poppeorum sp. nov. For the differences between $R$. tringa and these species, see respective Remarks sections.

\section{Habitat}

Intertidal to $72 \mathrm{~m}$, more frequently within the first $10 \mathrm{~m}$ of depth.

\section{Distribution}

New Caledonia, Vanuatu and Fiji (Fig. 5).

\section{Reticunassa visayaensis sp. nov. urn:1sid:zoobank.org:act:742DB3E4-4D0F-40DD-985C-948F113C4C65}

Fig. 3J-M, 6; Tables 1-2

Nassarius mamillatus - Martin 2008: 122, pl. 356, fig. 3 (non Preston). Nassarius pauperus - Martin 2008: 126, pl. 358, figs 1-4 (non Gould). 
Nassarius fuscolineatus - Martin 2008: 126, pl. 358, fig. 6 (non Smith).

\section{Etymology}

The name refers to the Visayas, a group of central Philippine Islands where this species is common.

\section{Type material}

Holotype

PHILIPPINES: lv, Panglao Island, $9^{\circ} 35.7^{\prime} \mathrm{N}, 123^{\circ} 44.7^{\prime} \mathrm{E}, 0-3 \mathrm{~m}$, seagrass and hard bottom, length $9.5 \mathrm{~mm}$, width $4.6 \mathrm{~mm}$, sequenced (MNHN IM-2007-31912).

\section{Paratypes}

PHILIPPINES: Panglao Island, Momo Beach, $9^{\circ} 36.1^{\prime} \mathrm{N}, 123^{\circ} 45.2^{\prime} \mathrm{E}, 0-3 \mathrm{~m}$ (MNHN IM-200028395, 9.2 mm; MNHN IM-2000 28405; MNHN IM-2000-28404, 3 spm); Panglao I. area (HD 24336, 60 spm; HK 184.13, 2 spm).

\section{Other material examined}

PHILIPPINES: PANGLAO 2004: Panglao I., stn B8, Napaling, 9³7.1' N, 12346.1' E, 3 m (2 spm); Panglao I., stn M1, Alona Beach, $9^{\circ} 32.9^{\prime} \mathrm{N}, 123^{\circ} 50.5^{\prime} \mathrm{E}, 5 \mathrm{~m}$ (2 spm); Panglao I., stn M5, Doljo Point, $9^{\circ} 35.5^{\prime} \mathrm{N}, 123^{\circ} 43.3^{\prime} / 123^{\circ} 44.3^{\prime} \mathrm{E}, 0-2 \mathrm{~m}$ (1 spm); Panglao I., stn M7, Momo Beach, $9^{\circ} 36.1^{\prime} \mathrm{N}$, $123^{\circ} 45.2^{\prime}$ E, 0-3 m (2 spm); Panglao I., stn M9, near Doljo Point, $9^{\circ} 35.1^{\prime} \mathrm{N}, 123^{\circ} 43.6^{\prime} \mathrm{E}, 0.5 \mathrm{~m}$ (1 spm); Panglao I., stn M10, Bingag/Tabalong, $9^{\circ} 37.8^{\prime} \mathrm{N}, 123^{\circ} 48.4^{\prime} \mathrm{E}, 0-3 \mathrm{~m}$ (1 spm); Panglao I., stn M22, Napaling, $9^{\circ} 37.2^{\prime} \mathrm{N}, 123^{\circ} 46.4^{\prime} \mathrm{E}, 0-3 \mathrm{~m}$ (1 spm); Panglao I., stn M40, Looc, $9^{\circ} 35.7^{\prime} \mathrm{N}, 123^{\circ} 44.7^{\prime} \mathrm{E}$, 0-3 m (1 spm); Panglao I., stn R19, Napaling, $9^{\circ} 37.1^{\prime} \mathrm{N}, 123^{\circ} 46.1^{\prime} \mathrm{E}, 2-54 \mathrm{~m}$ (2 spm); Panglao I., stn S24, Momo Beach, $9^{\circ} 36.1^{\prime} \mathrm{N}, 123^{\circ} 45.0^{\prime} \mathrm{E}, 2-4 \mathrm{~m}$ (1 spm); Pamilacan I., stn S42, 9³0.1' N, $123^{\circ} 55.5^{\prime} \mathrm{E}$, 15-20 m (1 spm). - Cebu, leg. R. Martin (ZMA.Moll.175495, $10 \mathrm{spm})$; Cebu (HK 184.09, $1 \mathrm{spm}$; ZMA Moll. 080981, 1 spm); Cebu, Liloan, 50-150 m, 2013 (HD 35803, 1 spm); Mactan I., Maribago, Buyon, in sandy tide pools, leg. O.K. McCausland (HK 184.07, 1 spm); Punta Engaño, in tangle nets, 150 m (HK 184.05, 3 spm); Punta Engaño, in coral rubble, 20 m (HK 184.14, 3 spm); Punta Engaño (HD 11610, 8 spm); Punta Engaño, 80-100 m (HD 17300, 3 spm); Mactan I., Punta Engaño, 25-50 m (HD 33879, 1 spm); Punta Engaño, leg. S.P. Kool, 2005 (HK 184.11, 1 spm); Punta Engaño, 1-2 m, live (HK 184.12, 2 spm); Punta Engaño, 25-30 m, 2009 (AMD, 2 spm); Punta Engaño, 100-150 m, 2009 (AMD, 2 spm); Balicasag I. (HD 15930, 2 spm); Olango I., night dive, 20-25 m (HD 17292, 3 spm); Olango I., 2014 (HD 35872, 4 spm); Olango I., 20-25 m, 2009 (AMD, 2 spm); Cuyo I., 15-20 m (HD 35488); Calituban I., 10 m (HK 184.01, 6 spm); SW side of Catanduanes, San Rafael, leg. S.P. Kool, 2005 (HK 184.10, 1 spm); Aliguay I., tangle nets, 150-180 m (HK 184.08, 2 spm); Palawan, 10-25 m, 2009 (AMD, 2 spm).

INDONESIA: SNELLIUS 1929: Ternate I., 1-2 Apr. 1930 (RMNH, 1 spm); Tidore I., 24-29 Nov. 1929 (RMNH, 1 spm). - RUMPHIUS 1990: Ambon, stn 05 Leitimur, Ambon Bay, outer bay, Tg. Benteng (RMNH, 2 spm); Ambon, stn 17, SE side of Pombo I. (RMNH, 2 spm); Ambon, stn 20 Hitu, N coast, Hitulama (RMNH, $1 \mathrm{spm}$ ); Ambon, stn 21 Hitu, N coast, Mamala (RMNH, $2 \mathrm{spm}$ ); Ambon, stn 23 Hitu, Kaitetu (near Hila), 22-23 Nov. 1990 (RMNH, 1 spm); Ambon, stn 26 Hitu, 4 km W of Kaitetu, 23 Nov. 1990 (RMNH, 3 spm); Ambon, stn 27 Leitimur, S coast, Hutumuri (RMNH, 1 spm); Ambon, stn 34 Hitu, Ruhmatiga 3-5 Dec. 1990 (RMNH, 4 spm; HK 184.03, 1 spm). - LAGON, stn Seith, Karubar, Amboine, low tide (1 spm); South Moluccas, leg. Rijkschroeff (ZMA.Moll.096203, ex coll. Butot 12403, 7 spm); South Moluccas, leg. Rijkschroeff (ZMA.Moll.096204, ex coll. Butot 12404, 5 spm); Ceram, N coast, Seleman Bay, leg. H. Strack (HK 184.04, 1 spm); Sulawesi, Lintido, leg. Semmelink (ZMA.Moll.096205, ex coll. Schepman, 1 spm); Bali, Sanur, leg. K. van Duin, 1989 (ZMA. Moll.099329, $1 \mathrm{spm}$ ); Bali, Kaliyasem, Lovina area, $8^{\circ} 09.7^{\prime} \mathrm{S}, 115^{\circ} 01.7^{\prime} \mathrm{E}, 1-2 \mathrm{~m}$ (HK 184.16, $\left.3 \mathrm{spm}\right)$; 
Flores, Labuan Bajo, Binongko Beach, leg. J.N.J. Post (HK 184.15, 1 spm); West Papua, Manokwari, near Uriami River, leg. D. Smits 1958/1961 (HK 184. 02, 11 spm; HD 38514, 11 spm).

PAPUA NEW GUINEA: PAPUA NIUGINI: stn PM22, Sek I., $05^{\circ} 04.7^{\prime} \mathrm{S}, 145^{\circ} 48.9^{\prime} \mathrm{E}, 0-1 \mathrm{~m}$ (MNHN IM-2013-13184; MNHN IM-2013-13192, 2 lv); stn PM19, Islet SE of Megas Islet, 0505.4' S, $145^{\circ} 48.6^{\prime}$ E, 0-1 m (2 spm); stn PB23, Lauhamug I., outer slope, 045․ $595^{\prime} \mathrm{S}, 145^{\circ} 47.7^{\prime} \mathrm{E}, 13 \mathrm{~m}$

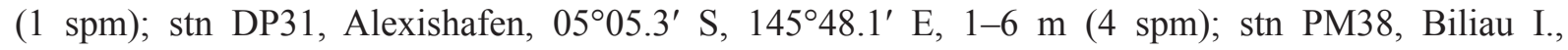
$05^{\circ} 11.8^{\prime} \mathrm{S}, 145^{\circ} 48.2^{\prime} \mathrm{E}$ (1 spm); stn PD78, Tabad I., 0508. $2^{\prime} \mathrm{S}, 145^{\circ} 48.7^{\prime} \mathrm{E}, 5 \mathrm{~m}$ (1 spm); stn PR202, $\mathrm{S}$ of Tab I., $05^{\circ} 10.3^{\prime} \mathrm{S}, 145^{\circ} 50.3^{\prime} \mathrm{E}, 2-4 \mathrm{~m}$ (2 spm).

\section{Description}

\section{Holotype}

Protoconch. Smooth, multispiral, whitish-yellowish, consisting of 2.5 whorls. Beset with rows of minute pustules (Fig. 3M).

SHELL. Elongate-ovate, 5.5 postnuclear whorls, suture impressed. Penultimate whorl with 15 nearly equally pronounced, round ribs; body whorl with 14 ribs, decreasing in height or disappearing on ventral side. Varix broad and strong.

SPIRAL CORDS. Continuous, flat and narrow, 7 cords on penultimate, and 11 on body whorl, peripheral one somewhat darker.

INTERCORDAL SCULPTURE. Approximately 6 very fine, evenly spaced striae between spiral cords.

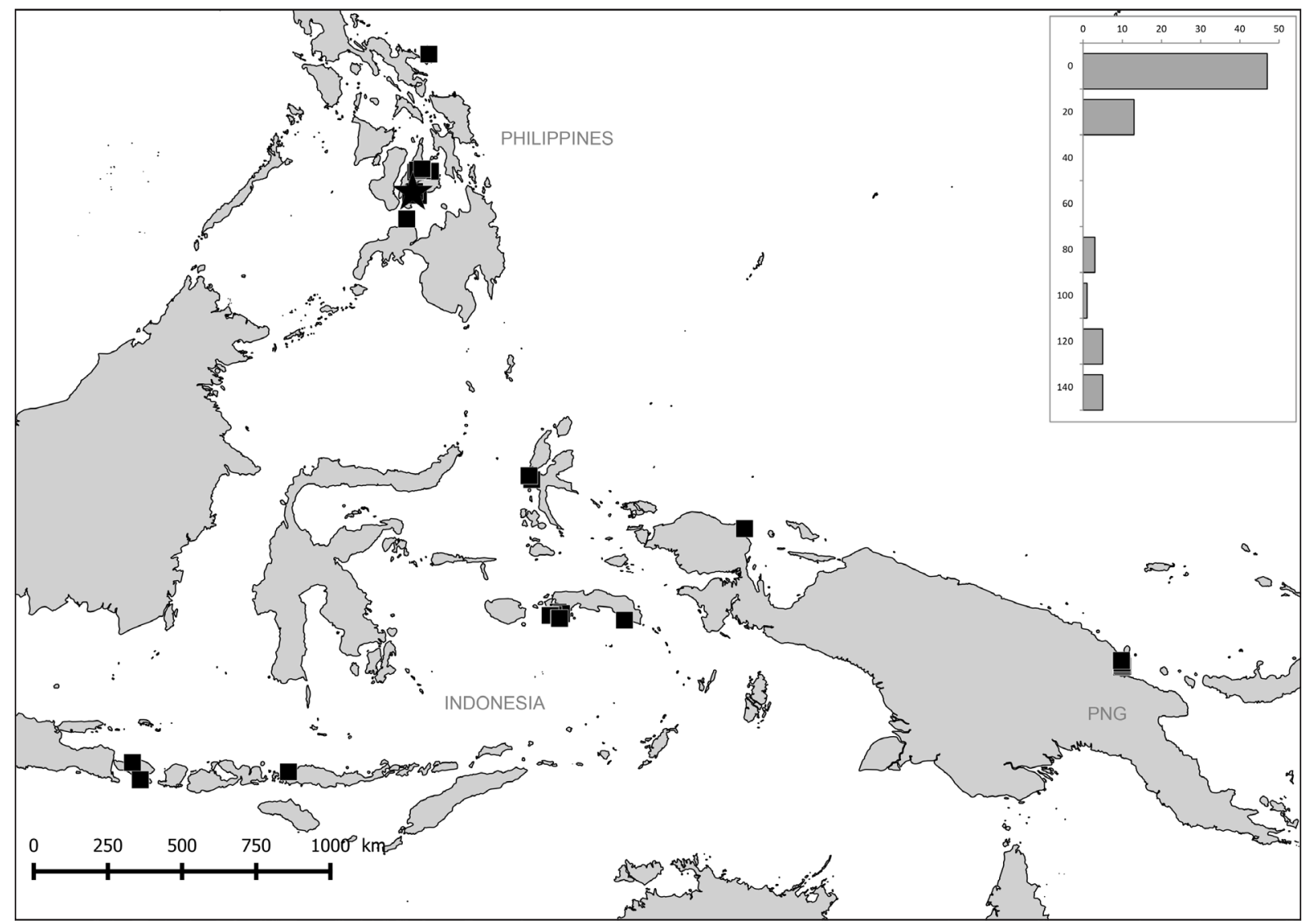

Fig. 6. Geographical and bathymetrical distribution of Reticunassa visayaensis sp. nov. Star indicates type locality. Each bar represents all lv or dd specimens. 
APERTURE. Oval, inside outer lip with 9 lirate denticles, peripheral denticle slightly more pronounced. Parietal denticle moderate, anal canal wide. Columellar callus wide, anteriorly somewhat elevated, posteriorly partly extending over whorl; well delineated border. Fine lirae over entire surface.

OPERCULUM. Yellowish, serrated.

SipHONAL CANAL. Fasciole strong, siphonal area with 1 strong and 5 weak cords.

CoLor. Yellowish, most spiral cords reddish between ribs.

Adult SIZE. $8.5-12.7 \mathrm{~mm}$, usually $8.5-10.5 \mathrm{~mm}$.

\section{Remarks}

The intraspecific variability is considerable. The number of ribs and spiral cords may vary, and the color is extremely variable, white to yellow and orange to brown, unicolor or with narrow or broad yellow, brown, dark brown, or grey bands on all whorls or only on the body whorl.

Reticunassa visayaensis sp. nov. has a broader, less pointed protoconch than $R$. paupera (Gould, 1850). $R$. visayaensis sp. nov. also differs from $R$. paupera by its larger size, its more bulbous shape and its lower ribs, especially on the body whorl, and by usually displaying dark bands.

$R$. visayaensis sp. nov. is very similar to $R$. tringa. When the protoconch is missing, a positive identification is almost impossible. The protoconch of $R$. tringa is paucispiral with $1.5-1.75$ whorls, whereas $R$. visayaensis sp. nov. has a protoconch of $2.25-2.5$ whorls. The protoconch of $R$. tringa is nipple-shaped, hence the name "mamillata" (Preston 1907); the protoconch of $R$. visayaensis sp. nov. is dome-shaped. Preston's description is accompanied by a drawing of the paucispiral protoconch.

R. visayaensis sp. nov. is the Reticunassa species most commonly offered in the shell trade from the central Philippines, as well as $R$. crenulicostata (Shuto, 1969). The latter is smaller $(5-7 \mathrm{~mm})$ and has a large multispiral protoconch of 3.5 whorls (Cernohorsky 1984: pl. 38, figs 1-2; Martin 2008: pl. 354, figs 4-5). These features are the most distinguishing differences between $R$. crenulicostata and $R$. visayaensis sp. nov. Reticunassa tringa has a paucispiral protoconch. Identification based on geographical distribution alone (Fig. 6) may be possible in specimens lacking a protoconch or welldefined teleoconch sculpture.

\section{Habitat}

Intertidal to $150 \mathrm{~m}$, commonly from 0 to $20 \mathrm{~m}$.

\section{Distribution}

Philippines, Indonesia and New Guinea (Fig. 6).

Reticunassa poppeorum sp. nov. urn:1sid:zoobank.org:act:21521D97-7E7D-4627-A806-43AF0E2AE7EA

Fig. 3N-Q, 7; Tables 1-2

\section{Etymology}

The name is given to honor Guido T. and Philippe Poppe for their many valuable contributions to the knowledge of and myriad publications on molluscs, including four volumes on Philippine marine molluscs and the magazine "Visaya." 
GALINDO L.A. et al., The Nassarius pauperus complex, part 3

\section{Type material}

\section{Holotype}

VANUATU: SANTO 2006, stn FB61, Aore Island, Port Benier, $15^{\circ} 34.4^{\prime} \mathrm{S}, 167^{\circ} 12.6^{\prime} \mathrm{E}, 2-3 \mathrm{~m}$, in fine coral sand (MNHN IM-2007-31779, lv, length $9.0 \mathrm{~mm}$, width $4.5 \mathrm{~mm}$ ).

\section{Paratypes}

VANUATU: SANTO 2006, stn DR03, Segond Channel, $15^{\circ} 31.0^{\prime} \mathrm{S}, 167^{\circ} 15.8^{\prime} \mathrm{E}, 2-30 \mathrm{~m}$, reef slope, fine rubble with Halimeda (MNHN IM-2007-32742, 1 lv); Éfate, snorkeling, 2-3 m, leg. T. McCleery, 2002 (HD 35802, 1 spm); Tutuba I., 15³2.3' S, 167²16.9’ E, 13 m (HK 137.23, 1 spm).

PHILIPPINES: PANGLAO 2004, stn S2, Bohol I., Baclayon, $9^{\circ} 37.4^{\prime} \mathrm{N}, 123^{\circ} 54.5^{\prime} \mathrm{E}, 4-5 \mathrm{~m}$ (MNHN IM-2007-31938, MNHN IM-2007-31937, 2 lv; HK 137.21, 2 spm); stn S15, Cortes Takot, 941.3' N, $123^{\circ} 49.5^{\prime}$ E, 4-6 m (MNHN IM-2000-22721, 1 lv; HK 137.01, 3 spm; HD 24148, 2 spm).

\section{Other material examined}

MALAYSIA: Sarawak, South China Sea, Muri I., off NW coast, 01 ${ }^{\circ} 54^{\prime} 13^{\prime \prime}$ N, $108^{\circ} 38^{\prime} 49.4^{\prime \prime}$ E, 10-30 m, leg. H. Morrison (GH, 1 spm); Sarawak, Miri, outer reef off Tanjung Lobang (JGMR, 1 spm); Sabah, Kota Kinabalu, Tanjung Aru (JGMR, 1 spm).

PHILIPPINES: PANGLAO 2004, stn S2, Bohol I., Baclayon, 9³7.4' N, 1235․ 5' E, 4-5 m (41 spm); stn S15, Bohol I., Cortes Takot, 941.3' N, 12349.5' E, 4-6 m (15 spm; ZMA.Moll.4.09.449, 1 spm);

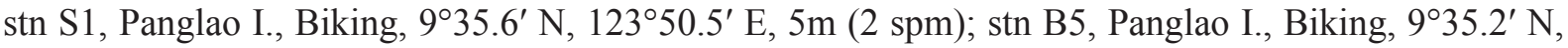
$123^{\circ} 50.4^{\prime}$ E, 4 m (2 spm); stn B8, Panglao I., Napaling, 9³7.1' N, 12346.1' E, 3 m (3 spm); stn S5,

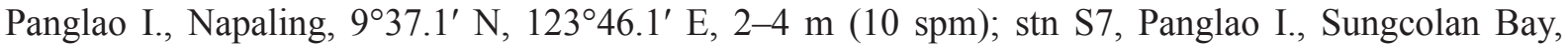

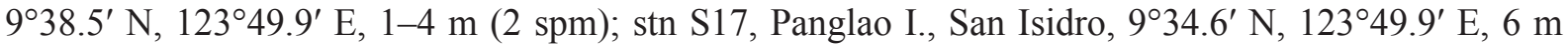
(1 spm); stn S24, Panglao I., Momo Beach, $9^{\circ} 36.1^{\prime} \mathrm{N}, 123^{\circ} 45.0^{\prime} \mathrm{E}, 2-4 \mathrm{~m}$ (1 spm); stn S32, Panglao

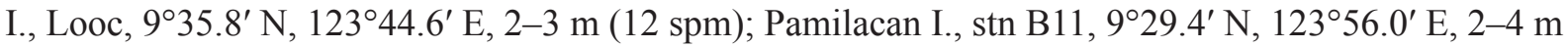
(1 spm); Pamilacan I., stn B24, 929.4' N, 12356.0' E, 38 m (2 spm); Pamilacan I., stn S10, 9²9.4' N, $123^{\circ} 56.0^{\prime}$ E, 6-14 m (2 spm); Pamilacan I., stn S14, 9²9.3' N, 123⒌ $55.1^{\prime}$ E, 5-12m (1 spm). - Cebu, leg. R. Martin (ZMA.Moll.175495, 2 spm); Cebu, by lumen lumen, 50-150 m (HK 137.19, 2 spm); Panglao I. area (HD 14809, 15 spm; HK 137.14, 1 spm); Siquijor I., tangle nets, 100 m (HD 17294, 1 spm); Balicasag I. (MUZEE, 2 spm); Mactan I., 10-30 m, 2012 (HD 28994, 7 spm); Mactan I., 20 m, 2014 (HD 35816, 1 spm); Olango I., 2014 (HD 35873, 1 spm); Camotes I., Santiago, 15 m, 2007 (HD 21366, 1 spm); Palawan I., 10-15 m, 2009 (HD 33880, 2 spm).

INDONESIA: Kalimantan, Berau Is, 02 ${ }^{\circ} 12^{\prime} 16.3^{\prime \prime}$ N, 118 35'18.9" E, 10-25 m (ZMA.Moll.175383, $1 \mathrm{spm}$ ); RUMPHIUS 1990, Ambon, stn 39 Hitu, W coast, S side of Larike (RMNH, 1 spm); Nusa Tenggara, Alor Strait, SE bay of Pantar I., 9-25 m, leg. H. Morrison (GH, 1 spm); Banda Sea, Wakatobi Group, E side of Moromaho I., 06 $08^{\prime} 59^{\prime \prime} \mathrm{S}, 124^{\circ} 35^{\prime} 11^{\prime \prime}$ E, leg. H. Morrison (GH, $6 \mathrm{spm}$ ); Flores Sea, $\mathrm{S}$ of Buton, 7-40 m, leg. H. Morrison (HK 137.08, 2 spm); N coast of Nusa Penida, leg. H. Morrison (GH, $1 \mathrm{spm}$ ); NW side of Sulawesi, Makassar Strait, Tambu Bay, sand and rubble slope, 10-40 m, among gorgonian corals, $00^{\circ} 01^{\prime} 07^{\prime \prime} \mathrm{S}, 119^{\circ} 43^{\prime} 77^{\prime \prime} \mathrm{E}$ (GH, $6 \mathrm{spm}$ ); Karang Kapota Atoll, SE of Buton I., 5 $5^{\circ} 7^{\prime} 73^{\prime \prime} \mathrm{S}, 123^{\circ} 24^{\prime} 10^{\prime \prime} \mathrm{E}$, on ledges of wall, 10-40 m, leg. H. Morrison (GH, $\left.1 \mathrm{spm}\right)$; Berau I., W side of Maratua I., 02 $12^{\prime} 16.3^{\prime \prime}$ N, 118 $35^{\prime} 18.9^{\prime \prime}$ E, 10-25 m (ZMA.Moll.175383, $1 \mathrm{spm}$ ); near Kalimantan, Pejantan I., $0^{\circ} 30^{\prime} 48.8^{\prime \prime}$ N, 107 $13^{\prime} 24^{\prime \prime}$ E, 10-20 m, leg. H. Morrison (GH, 1 spm); sand, near wreck of Japanese fighter, $0^{\circ} 45^{\prime} 16^{\prime \prime} \mathrm{N}, 105^{\circ} 36^{\prime} 11^{\prime \prime} \mathrm{E}, 45 \mathrm{~m}$, leg. H. Morrison (GH, $\left.2 \mathrm{spm}\right)$; near wreck, Acasta Rock, 01 ${ }^{\circ} 39^{\prime} 12^{\prime \prime} \mathrm{N}, 106^{\circ} 18^{\prime} 26^{\prime \prime} \mathrm{E}, 9-50 \mathrm{~m}$ (GH, $\left.1 \mathrm{spm}\right)$; sand, near wreck, 0 $45^{\prime} 15^{\prime \prime} \mathrm{N}$, $105^{\circ} 36^{\prime} 11^{\prime \prime}$ E, leg. H. Morrison (GH, 2 spm); Badas I. Group, 0³3'8.9" N, 106 58'47.4" E, 8-30 m, leg. H. Morrison (GH, $1 \mathrm{spm}) ; 01^{\circ} 38^{\prime} 7^{\prime \prime} \mathrm{N}, 106^{\circ} 21^{\prime} 23^{\prime \prime} \mathrm{E}$, on reef, 10-25 m, leg. H. Morrison (GH, 1 spm); Sebangmawang I., S of Bunguran I., 03 $33.92^{\prime}$ N, 108 $02.08^{\prime}$ E, 10-15 m, leg. H. Morrison 
(GH, 4 spm); Sumatra, Kelulekabung I. (HK 137.07, 1 spm); West Papua, Manokwari, mouth of Uriami River, leg. D. Smits (HK 137.06, 2 spm); Bay of Hollandia, leg. Mrs H. Grootens-Boerefijn (HK 137.11, $6 \mathrm{spm}$ ); off NW coast, Waigeo I., Sorong, leg. H. Morrison (HK 137.04, 2 spm); off Sorong, Kri I., 10-15 m, leg. H. Morrison (HK 137.05, 2 spm).

PAPUA NEW GUINEA: PAPUA NIUGINI: stn PB18, Sek I., outer slope, $05^{\circ} 06.3^{\prime} \mathrm{S}, 145^{\circ} 49.1^{\prime} \mathrm{E}$,

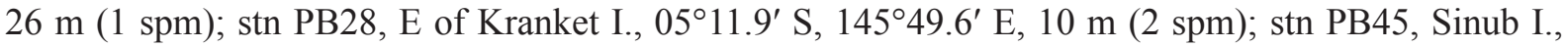
$05^{\circ} 07.9^{\prime} \mathrm{S}, 145^{\circ} 48.9^{\prime} \mathrm{E}, 8 \mathrm{~m}$ (4 spm); stn PB47, N of Kranket I., $05^{\circ} 11.3^{\prime} \mathrm{S}, 145^{\circ} 49.6^{\prime} \mathrm{E}, 5 \mathrm{~m}$ (MNHN

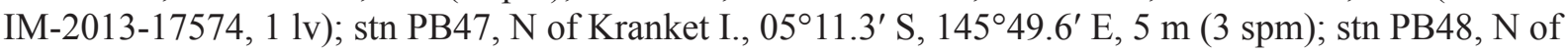

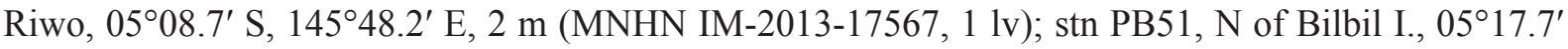

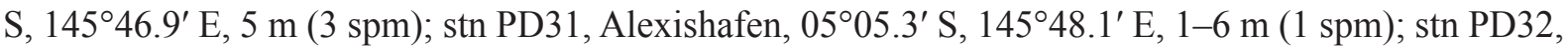
$\mathrm{N}$ of Sek I., Ottilien Passage, $05^{\circ} 04.4^{\prime} \mathrm{S}, 145^{\circ} 48.7^{\prime} \mathrm{E}, 1-8 \mathrm{~m}$ (1 spm); stn PD36, Rempi Area, W of Barag I., 0501.2' S, $145^{\circ} 47.9^{\prime} \mathrm{E}, 5-10 \mathrm{~m}$ (1 spm); stn PM19, Alexishafen, islet SE of Megas Islet,

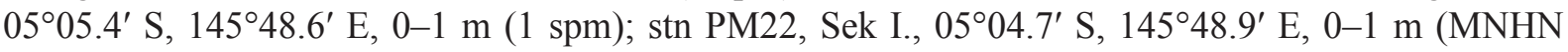

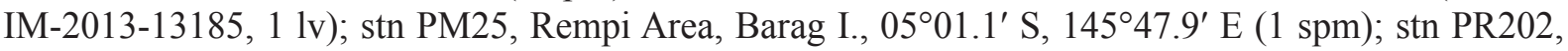
S of Tab I., $05^{\circ} 10.3^{\prime} \mathrm{S}, 145^{\circ} 50.3^{\prime} \mathrm{E}, 2-4$ m (MNHN IM-2013-17555, IM-2013-17556, IM-2013-17557, IM-2013-17558, IM-2013-17559, IM-2013-17560, 6 lv); stn PS04, Tab I., 05¹0.0' S, $145^{\circ} 50.1^{\prime}$ E,

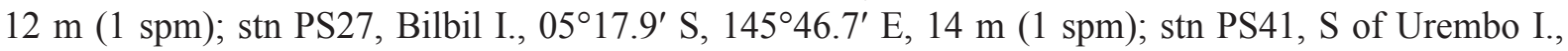
outer slope, $05^{\circ} 15.9^{\prime} \mathrm{S}, 145^{\circ} 47.1^{\prime} \mathrm{E}, 10 \mathrm{~m}$ (HK 137.20, $3 \mathrm{spm}$ ); stn PS45, W Yabob I., 05 $15.4^{\prime} \mathrm{S}$, $145^{\circ} 47.0^{\prime}$ E, 6 m (MNHN IM-2013-17562, 1 lv); stn PS47, N of Sek I., inner slope, $05^{\circ} 04.7^{\prime}$ S, $145^{\circ} 48.9^{\prime}$ E, $8 \mathrm{~m}$ (1 spm). - Bismarck Archipelago, New Ireland, Kavieng, Baudisson Bay, 15-35 m, leg. H. Morrison (HK 137.03, 2 spm).

SOLOMON ISLANDS: SALOMON 2, stn DW2234, Choiseul I., 651' S, 156 $24^{\prime}$ E, 182-277 m (1 spm); SALOMONBOA 3, stn DW2827, N of San Cristobal, $10^{\circ} 26^{\prime} \mathrm{S}, 161^{\circ} 51^{\prime} \mathrm{E}, 134-272 \mathrm{~m}$ (2 spm).

FIJI: Suva Point, leg. S.P. Kool (HK 137.15, 1 spm); Yasawa Group, Wayasewa I., 17²1' S, $177^{\circ} 08^{\prime}$ E, intertidal (HK 137.17, $1 \mathrm{spm}$ ).

SAMOA: Savaii I., Asau Bay (PS, 4 spm; HK 137.10, 2 spm).

\section{Description}

\section{Holotype}

Protoconch. Multispiral consisting of 2.5 whitish whorls, body whorl with rows of microscopic pustules, otherwise smooth (Fig. 3Q).

SHELL. Teleoconch consisting of 5.5 whorls; penultimate whorl with 15 and body whorl with 12 rounded ribs of equal size; broad varix.

SPIRAL CORDS. Body whorl with approximately 10 continuous, weak spiral cords.

INTERCORDAL SCULPTURE. Very fine, evenly spaced spiral striae, occasionally not only between, but also on spiral cords.

APERTURE. Six low denticles on outer lip, that at periphery more pronounced. Columellar callus elevated anteriorly, backside of elevation with microscopic growth lines. Callus partly extending posteriorly over body whorl onto anal canal. Columellar lip lirate onto moderately strong parietal denticle; anal canal deep.

Operculum. Cream colored, serrated. 
SiPHONAl CANAL. Narrow. A strong fasciole; siphonal area with several fine, one dominating, cords.

CoLOR. Whitish to yellowish; some spiral cords light brown between ribs. Lower half of dorsal side of body whorl frequently with broad, orange-brown band (Fig. 3O).

AdULT SIZE. 6.9-12.0 mm.

\section{Remarks}

The color varies from white to yellow and orange; the spiral cords occasionally brown, sometimes only locally between the axial ribs. Some specimens have 2 brown bands on the body whorl, occasionally only visible on the varix. Some specimens, including the holotype, display a broad orange-brown band on the lower part of the body whorl. $R$. poppeorum sp. nov. is often recognizable by a broad, dark orange-brown band on the last half or the last third of the ultimate whorl, and is characterized by its more slender shape compared to other species in this genus.

Reticunassa poppeorum sp. nov. shows resemblance to $R$. tringa and $R$. visayaensis $\mathrm{sp}$. nov. The greatest difference among the three species is the morphology of the protoconch. $R$. tringa has a paucispiral protoconch with $1.5-1.75$ whorls, $R$. visayaensis sp. nov. has a multispiral protoconch of $2.25-2.5$ whorls, whereas the protoconch of $R$. poppeorum sp. nov. has $2.5-2.75$ whorls. The axial ribs of $R$. poppeorum sp. nov. are more pronounced and are present on the entire body whorl, whereas they decrease in height on the ventral side of the body whorl in $R$. tringa and $R$. visayaensis sp. nov.

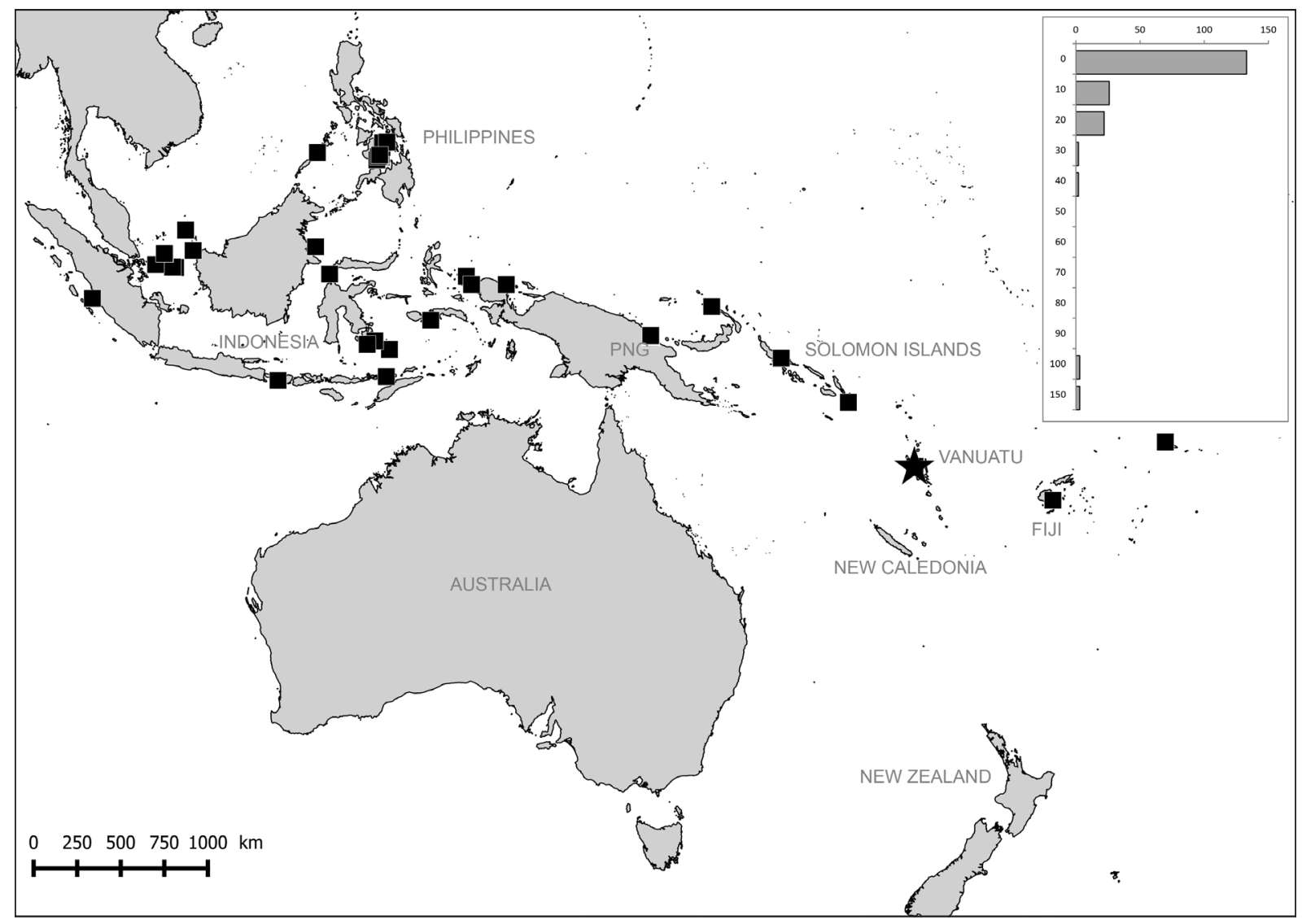

Fig. 7. Geographical and bathymetrical distribution of Reticunassa poppeorum sp. nov. Star indicates type locality. Each bar represents all lv or dd specimens. 
The most noticeable difference between $R$. paupera on the one hand and $R$. tringa, $R$. visayaensis $\mathrm{sp}$. nov. and R. poppeorum sp. nov. on the other, is the presence of lirae in the latter three species over the entire surface of the extended columella. Besides this sculptural feature, the shape of the columellar callus differentiates $R$. paupera from the other three species; the callus of $R$. paupera is practically limited to the columella, whereas the callus of the other three partly extends over the body whorl.

There are no morphological differences between R. neoproducta Kool \& Dekker, 2007 and the closely related R. poppeorum sp. nov. (Fig. 3N-Q). However, we decided to considerer these two lineages as different species based on the reciprocal monophyly indicated by the taxonomic trees (COI and $28 \mathrm{~S}$ separately). Furthermore, $R$. neoproducta is found in the western Indian Ocean and $R$. poppeorum occurs in the western Pacific Ocean. To date, no specimens of these two species have been found east of Sri Lanka or west of Sumatra, respectively. It seems very unlikely that these two allopatric lineages still share any gene flux.

\section{Habitat}

In sand and coral rubble, 0-40 m, mainly between 1 to $20 \mathrm{~m}$. Some freshly dead specimens with operculum were collected on a hard bottom with small pockets of sediment and in coral sand at a depth of 4-6 m. Some specimens were collected in mangrove areas and in seagrass.

\section{Distribution}

Malaysia, Indonesia, the Philippines, Fiji, and Western Samoa (Fig. 7).

Reticunassa annabolteae sp. nov. urn:1sid:zoobank.org:act:781F25AD-B216-47A1-8084-FC46ED4C8029

Fig. 8A-D, 9; Tables 1-2

\section{Etymology}

The species is dedicated to Mrs Anna Bolte, the second author's partner and travel companion for more than 20 years.

\section{Type material}

\section{Holotype}

MADAGASCAR: ATIMO VATAE, stn BM06, South Madagascar, Ambatobe, Bavarama, 25⒉ $29^{\prime}$ S, $44^{\circ} 57.6^{\prime} \mathrm{E}, 0-1 \mathrm{~m}$, in rolling stones (MNHN IM-2009-12843, lv, sequenced, length $11.5 \mathrm{~mm}$, width $5.3 \mathrm{~mm})$.

\section{Paratypes}

MADAGASCAR: same collection data as holotype (MNHN IM-2009-12812, MNHN IM-2009-12849, MNHN IM-2009-12862, MNHN IM-2009-12868, 4 lv; HK 302.01, 3 spm; HD 37306, 2 spm); S coast, Lavanono (HK 302.05, 5 spm; HK 302.06, 2 spm).

\section{Other material examined}

MADAGASCAR: ATIMO VATAE, South Madagascar: stn BB03, Lavanono, 25⒉ $26.4^{\prime} \mathrm{S}, 4^{\circ} 56.1^{\prime} \mathrm{E}$,

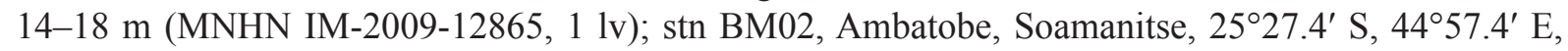

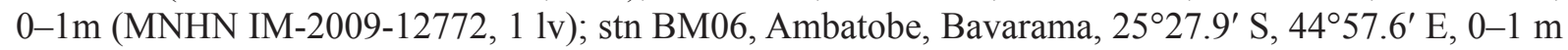
(MNHN IM-2009-12778, MNHN IM-2009-12783, MNHN IM-2009-12825, MNHN IM-2009-12841, MNHN IM-2009-12844, 5 lv); stn BM09, Saririaka, 2529.0' S, 4459.6' E, 0-1 m (MNHN IM-2009-

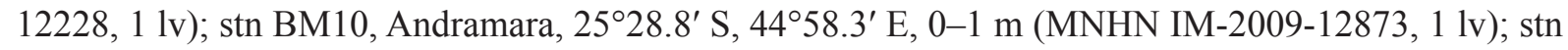




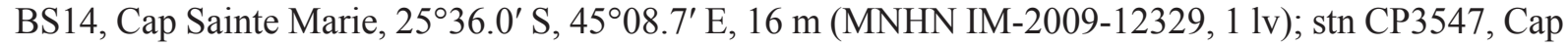
Andavaka, 25⒙0' S, 46 40.3' E, 69-70 m (MNHN IM-2009-12893, 1 lv); stn TM01, Monseigneur

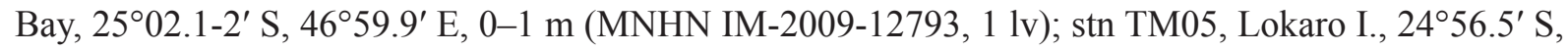
4707.1' E, 0-1 m (MNHN IM-2009-12306, MNHN IM-2009-12888, MNHN IM-2009-12889, MNHN IM-2009-12897, MNHN IM-2009-12899, MNHN IM-2009-12905, MNHN IM-2009-13087, 7 lv); stn

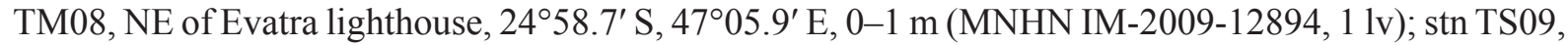

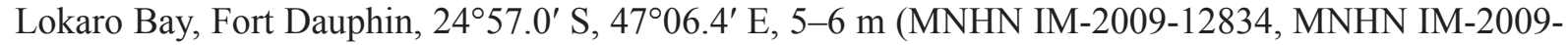

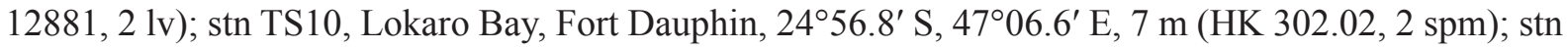
TS21, Phare Flacourt, $25^{\circ} 01.5^{\prime} \mathrm{S}, 47^{\circ} 00.0^{\prime}$ E, 2-4 m (HK 302.03, 2 spm); stn TV07, Phare Flacourt,

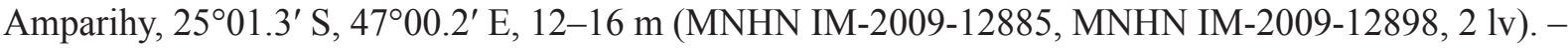
South Madagascar (HK 302.04, 1 spm); Lavanono (HD 21156, 2 spm); Lavanono (HD 36646, $21 \mathrm{spm}$ ); Soamanitse (HD 20287, $3 \mathrm{spm}$ ).

\section{Description}

\section{Holotype}

Protoconch. Paucispiral consisting of $1.5-1.75$ broad, flattened, axially-striated whorls. Separation between protoconch and teleoconch indistinct (Fig. 8D).

SHELl. Elongate-ovate, consisting of 5.5 convex whorls, suture deep; axial ribs poorly developed, decreasing in height on body whorl; 16 ribs on penultimate whorl and 14 on body whorl; broad varix.

SPIRAL CORDS. Fine, weak compared to other species in genus, more pronounced where they cross axial ribs. Two anterior spiral cords more developed, resulting in two deep spiral grooves.

INTERCORDAL SCULPTURE. Very fine spiral striae between spiral cords.

APERTURE. Oval, inside outer lip 8 lirate denticles; columellar callus plicate throughout, somewhat elevated anteriorly, posteriorly slightly extended over body whorl; parietal denticle weak, anal canal wide; siphonal area with 4-5 low cords.

OPERCULum. Yellowish, serrated.

Siphonal Canal. Deep and narrow. Fasciole deep.

CoLoR. Yellow-orange, narrow brown band above suture and broad brown band below periphery. Most spiral cords darker.

AduLt SIZE. 7.5-12.6 mm, usually 9-11 mm.

\section{Remarks}

Besides the variability in color, from off-white to yellowish-orange, the difference in size of adult specimens is remarkable. Some specimens have somewhat more developed spiral cords.

Comparison with some other Reticunassa species with a paucispiral protoconch: $R$. tringa is a smaller, more bulbous species with a pointed protoconch; $R$. goliath sp. nov. also has fine spiral cords as found in $R$. annabolteae sp. nov., but differs in its pointed protoconch; $R$. intrudens sp. nov. has a protoconch intruded by the teleoonch spiral cords; R. zanzibarensis (Kool \& Dekker, 2007) has a smooth protoconch contrasting the axially-striated protoconch in $R$. annabolteae sp. nov. 


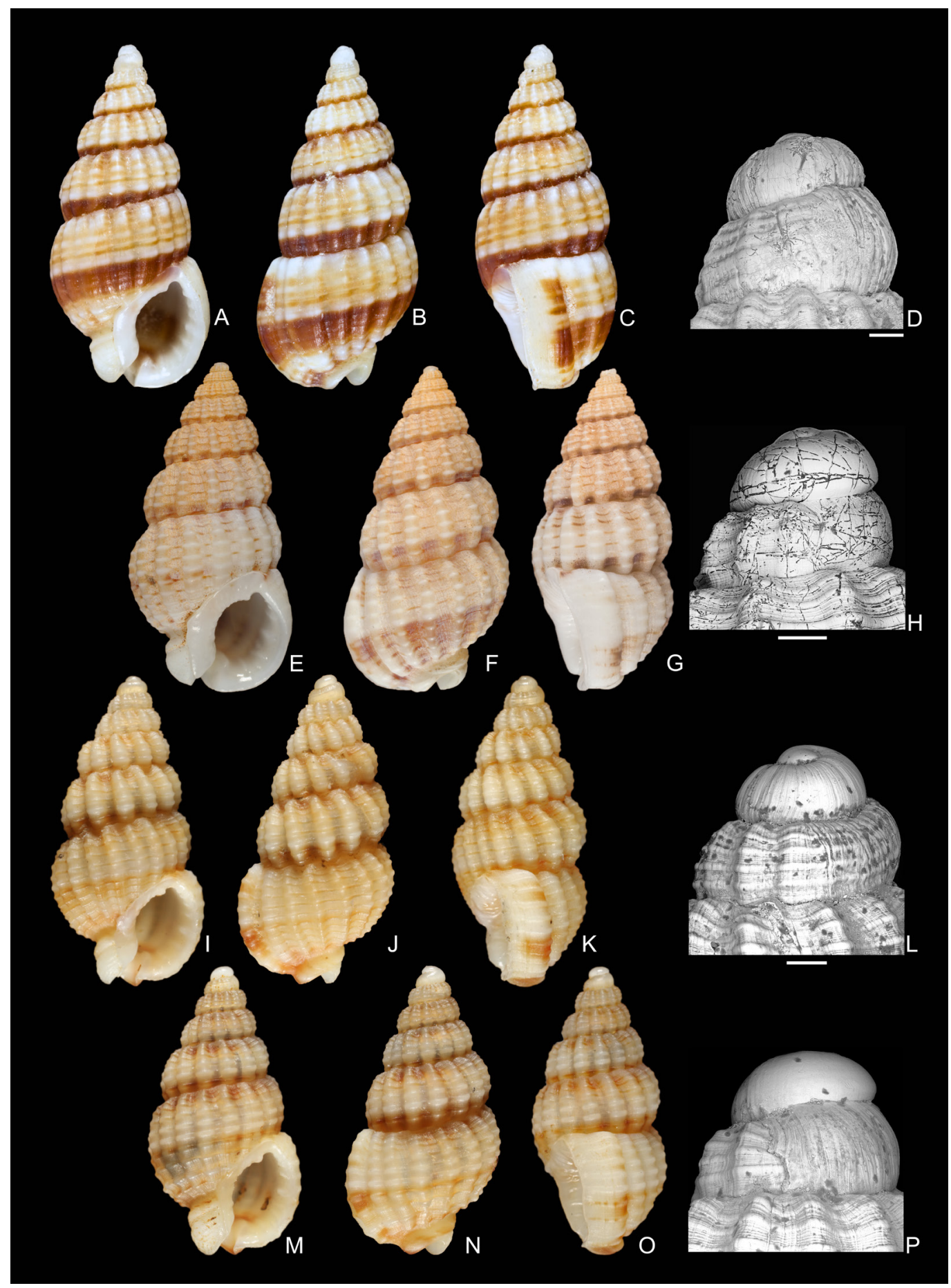

Fig. 8. Shells of Reticunassa Iredale, 1936. - A-D. Reticunassa annabolteae sp. nov. A-C. Holotype, MNHN IM-2009-12843, length $11.5 \mathrm{~mm}$, width $5.3 \mathrm{~mm}$, S Madagascar, Ambatobe, Bavarama, 25⒉ $2.9^{\prime} \mathrm{S}$, 445․ $6^{\prime}$ E, 0-1 m. D. Protoconch. - E-H. R. goliath sp. nov. E-G. Holotype, MNHN IM-200022724, length $15.5 \mathrm{~mm}$, width $7.9 \mathrm{~mm}$, New Caledonia, Nouméa, leg. D. Massemin, ex coll. H. Kool. H. Protoconch of paratype, MNHN IM-2000-28394. - I-L. R. intrudens sp. nov. Holotype, MNHN IM-2000-22726, length $7.9 \mathrm{~mm}$, width $4.3 \mathrm{~mm}$, New Caledonia, Touho, 204' $\mathrm{S}, 165^{\circ} 14^{\prime} \mathrm{E}, 49-59 \mathrm{~m}$. L. Protoconch. - M-P. R. thailandensis sp. nov. M-O. Holotype, ZMA Moll.4.09.047, length $7.35 \mathrm{~mm}$, width $3.8 \mathrm{~mm}$, Thailand, Gulf of Thailand, $6^{\circ} 40.313^{\prime} \mathrm{N}, 101^{\circ} 43.852^{\prime} \mathrm{E}, 3-15 \mathrm{~m}$. P. Protoconch. Scale bars: $\mathrm{D}, \mathrm{H}, \mathrm{L}, \mathrm{P}=200 \mu \mathrm{m}$. 


\section{Habitat}

Intertidal to $70 \mathrm{~m}$, frequently between 0 and $10 \mathrm{~m}$. Some specimens were found living on rocky platforms with algae, associated to eunicid tubes, coarse sand, and rolling stones.

\section{Distribution}

Only known from southern Madagascar (Fig 9).

Reticunassa goliath sp. nov. urn:Isid:zoobank.org:act:5C34E90F-3AD2-42ED-B7A6-582525AD11DB

Fig. $8 \mathrm{E}-\mathrm{H}, 10$; Table 2

\section{Etymology}

This species is named after the biblical Goliath, the giant who fought against David. This name is chosen because Reticunassa goliath sp. nov. is to date the largest known species in the genus Reticunassa.

\section{Type material}

\section{Holotype}

NEW CALEDONIA: Nouméa, in sandy areas within coral reef, snorkeling at night, leg. D. Massemin (MNHN IM-2000-22724, ex coll. H. Kool, length 15.4 mm, width 7.9 mm).

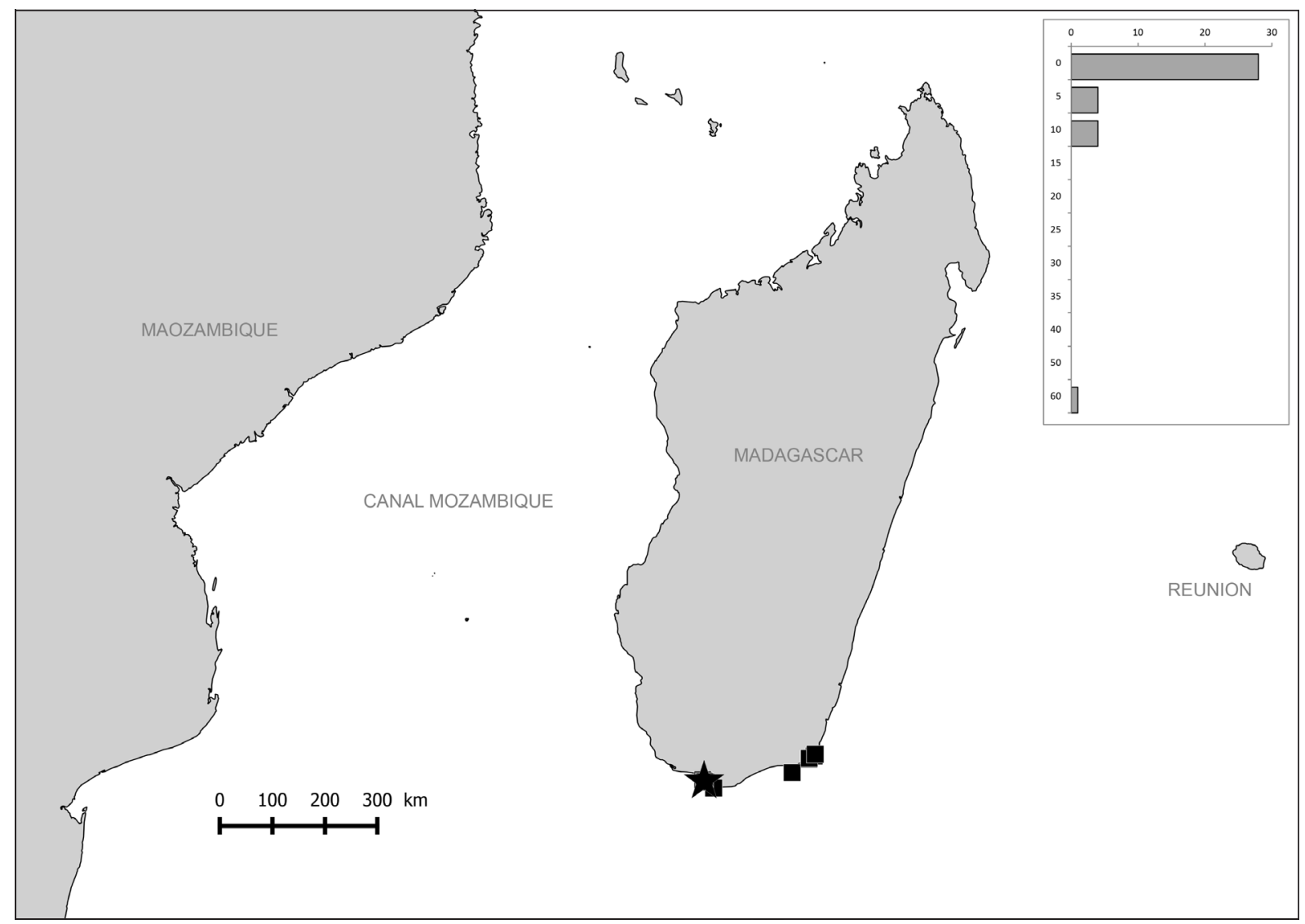

Fig. 9. Geographical and bathymetrical distribution of Reticunassa annabolteae sp. nov. Star indicates type locality. Each bar represents all lv or dd specimens. 
Paratypes

NEW CALEDONIA: PLOUVEAL, stn 1221, Lagon d'Ouvéa, 20²9' S, 166³1' E, 10 m (MNHN IM2000-22725, MNHN IM-2000-22723, MNHN IM-2000-28394, 3 spm; ZMA.Moll.4.09.050, 1 spm; GH, 1 spm; HD 24143, 2 spm; HK 997.01, 3 spm; HUJ, 1 spm); PLOUVEAL, stn 1227, 12³7' S, 166 25' E, 12 m (HD 35304, 2 spm; HK 997.05, 2 spm); Nouméa, sand between coral reefs, snorkeled at night (HK 997.07, $1 \mathrm{spm}$ ); Nouméa, Nouville Beach, brown sand, $10 \mathrm{~m}$ (HK 997.08, $1 \mathrm{spm}$ ); New Caledonia, 5-10 m (HD 26939, $1 \mathrm{spm}$ ).

\section{Other material examined}

INDONESIA: West Papua, Manokwari, leg. D. Smits (HK 997.03, 2 spm).

AUSTRALIA: North Queensland, Bowen, Grey’s Bay, ex coll. Hessel (ZMA, 4 spm; HK 997.06, 1 spm).

VANUATA: SANTO 2006, stn FB52, Malokilikili, 1542.7' S, 167²15.1' E, 7 m (1 spm).

NEW CALEDONIA: LAGON: stn 161, Ile Ouen-Baie du Prony, 22 $2^{\circ} 34^{\prime}$ S, $166^{\circ} 38^{\prime}$ E, 20 m (1 spm); stn 943, Koumac, $20^{\circ} 37^{\prime} \mathrm{S}, 164^{\circ} 11^{\prime} \mathrm{E}, 15 \mathrm{~m}$ (1 spm). - PLOUVEAL, Lagon d'Ouvéa: stn 1218, 20³6' S, $166^{\circ} 30^{\prime} \mathrm{E}, 13 \mathrm{~m}(8 \mathrm{spm})$; stn $1219,20^{\circ} 30^{\prime} \mathrm{S}, 166^{\circ} 28^{\prime} \mathrm{E}, 15 \mathrm{~m}$ (26 spm); stn 1220, $20^{\circ} 29^{\prime} \mathrm{S}, 1^{\circ} 6^{\circ} 29^{\prime} \mathrm{E}$,

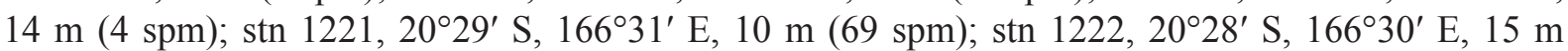

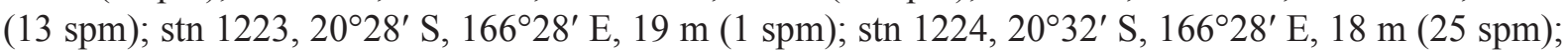

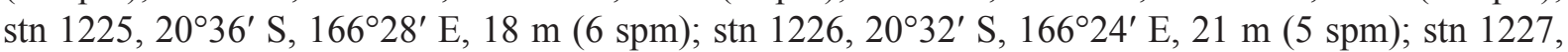
$12^{\circ} 37^{\prime} \mathrm{S}, 166^{\circ} 25^{\prime} \mathrm{E}, 12 \mathrm{~m}$ (29 spm); stn $1228,20^{\circ} 36^{\prime} \mathrm{S}, 166^{\circ} 24^{\prime} \mathrm{E}, 18 \mathrm{~m}(8 \mathrm{spm})$; stn $1229,20^{\circ} 37^{\prime} \mathrm{S}$,

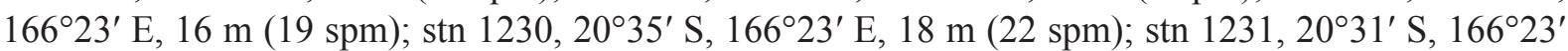

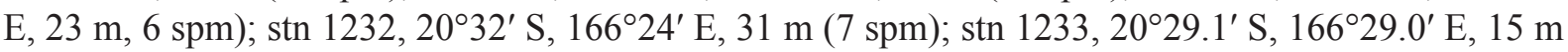

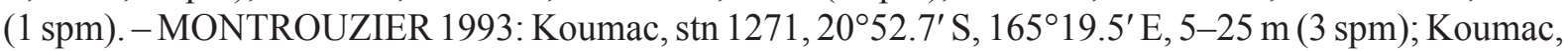
stn 1286, Plateau Karembé, $20^{\circ} 38^{\prime}-20^{\circ} 39^{\prime}$ S, $164^{\circ} 16^{\prime}-164^{\circ} 17^{\prime}$ E (3 spm); Koumac, stn 1287, Récif de

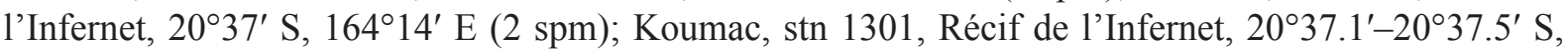
$164^{\circ} 14.7^{\prime}-164^{\circ} 15^{\prime}$ E, $1-5 \mathrm{~m}$ (3 spm); Koumac, stn 1303, Lagon, parages du Plateau Karembé, $20^{\circ} 37.7^{\prime}-$ $20^{\circ} 38.8^{\prime} \mathrm{S}, 164^{\circ} 15.9^{\prime}-164^{\circ} 17.1^{\prime} \mathrm{E}, 0-8 \mathrm{~m}$ (2 spm); Koumac, stn 1304, Chenal de l'Infernet, $20^{\circ} 38.6^{\prime} \mathrm{S}$, $164^{\circ} 13.2^{\prime} \mathrm{E}, 12-15 \mathrm{~m}$ (2 spm); Koumac, stn 1306 , Chenal de l'Infernet, $20^{\circ} 39.1^{\prime} \mathrm{S}, 1^{\circ} 4^{\circ} 12.4^{\prime} \mathrm{E}, 11-13 \mathrm{~m}$

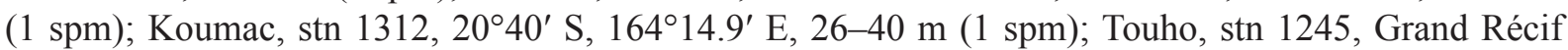
Mengalia, $20^{\circ} 45.2^{\prime} \mathrm{S}, 165^{\circ} 16.3^{\prime} \mathrm{E}$ (4 spm); Touho, stn 1273, Touho region, outer reef, $20^{\circ} 50.4^{\prime} \mathrm{S}$, $165^{\circ} 22.8^{\prime} \mathrm{E}, 20 \mathrm{~m}(2 \mathrm{spm})$. - PALEO-SURPRISE 1999, stn CP1388, $18^{\circ} 23.8^{\prime} \mathrm{S}, 1^{\circ} 3^{\circ} 06.9^{\prime} \mathrm{E}, 40 \mathrm{~m}$ $(2 \mathrm{spm})$.

LOYALTY ISLANDS: LIFOU 2000, stn 1419, Lifou, Santal Bay, Bay of Gaatcha, 20 $55.6^{\prime} \mathrm{S}, 167^{\circ} 04.5^{\prime} \mathrm{E}$, 5 m (8 spm; HK 997.02, 1 spm); LIFOU 2000, stn 1426, Lifou, Santal Bay, $20^{\circ} 45.9^{\prime}$ S, $167^{\circ} 06.2^{\prime}$ E, $4-7$ m (2 spm); Lifou, leg. J.R. le B. Tomlin (ZMA.Moll.096228, ex coll. Schepman, 6 spm); Lifou, leg. R.P. Goubin (ZMA.Moll.089599, ex coll. Dautzenberg, 3 spm); Lifou (HUJ 9055, ex coll. Dautzenberg, ex coll. Coen 7553, $3 \mathrm{spm})$.

\section{Description}

\section{Holotype}

Protoconch. Paucispiral, pointed, 1.75 milky whorls, beset with rows of microscopic pustules. Protoconch missing in holotype, seen in paratype (Fig. 8H).

SHELl. Rather heavy, conical, acuminate, teleoconch consisting of 7 whorls, suture impressed. On penultimate 13 and on body whorl 12 well pronounced, equidistant axial ribs and strong varix. Ribs equally strong on dorsal and ventral side of body whorl. 
SPIRAL CORDS. Continuous, moderately broad on top of axial ribs, but between ribs narrower and weak, approximately 6 cords on penultimate and 10 on body whorl.

INTERCORDAL SCULPTURE. Numerous very fine, evenly spaced spiral striae, occasionally also on top of spiral cords.

APERTURE. Round, proportionally small, approximately $1 / 5$ of shell length. Outer lip with 7 unequal denticles. Columella callused, somewhat elevated anteriorly and with evenly spaced lirae throughout. Callus sharply bordered, bending over part of fasciole and somewhat extending over body whorl posteriorly. Inside outer lip with 8 lirae and pronounced tooth at siphonal canal. Elevated part of callus with fine growth lines on left (out)side. Parietal denticle strong, anal canal deep.

OperCulum. Yellowish, serrated.

SiPHONAl CANAL. Narrow, fasciole strong. Siphonal area with one strong and some weak cords.

COLOR. Off white to yellow, narrow band just above suture and broader band below periphery; columella and outer lip white, aperture yellowish.

AduLt SIZE. $8.2-16.5 \mathrm{~mm}$, usually $13-15 \mathrm{~mm}$.

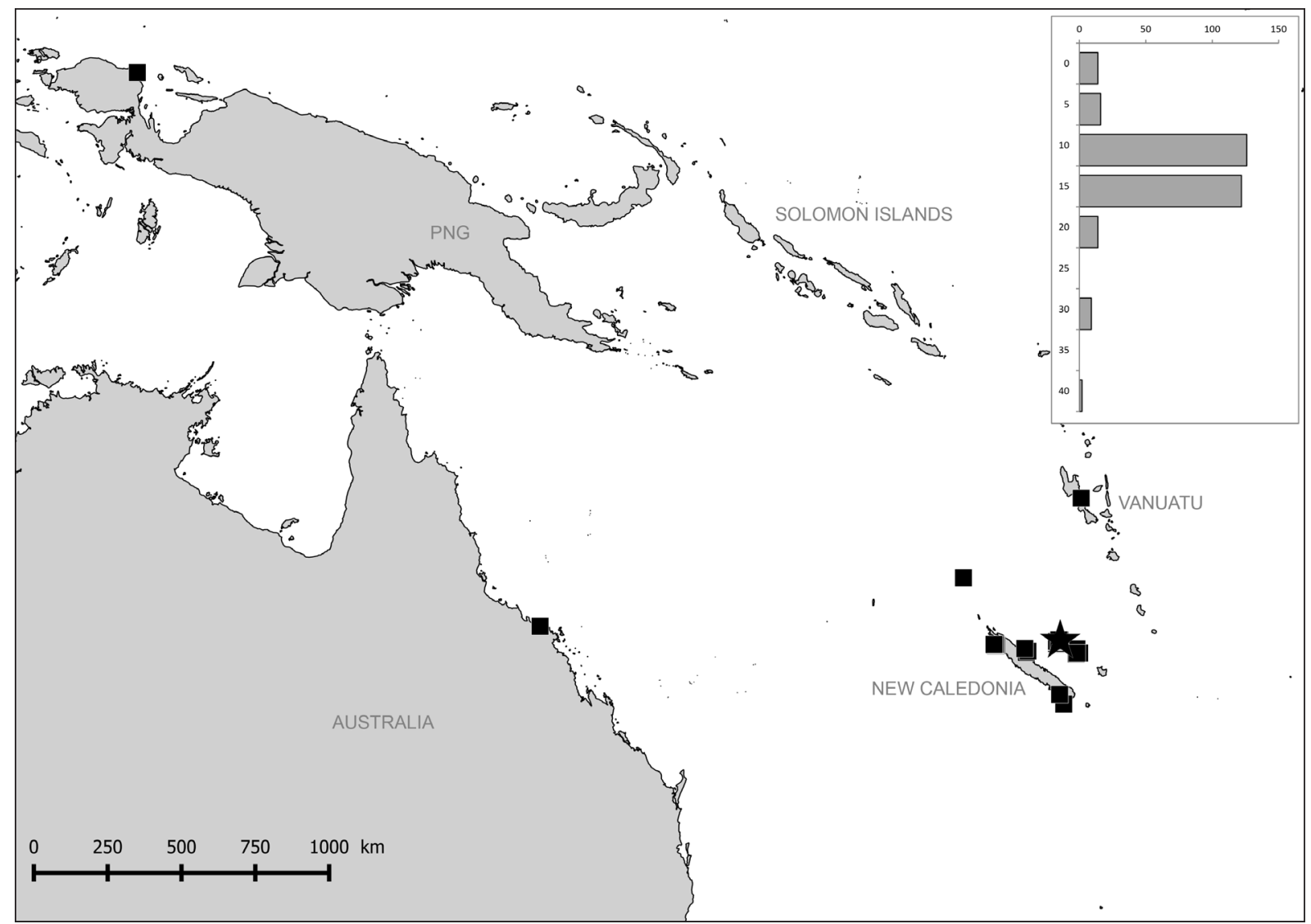

Fig. 10. Geographical and bathymetrical distribution of Reticunassa goliath sp. nov. Star indicates type locality. Each bar represents all lv or dd specimens. 


\section{Remarks}

Color and banding pattern variable; some specimens have light to dark brown bands and others lack banding. In banded specimens, one band occurs on the body whorl, with an additional band on the sutural area. In some specimens, the spiral cords may be colored orange/brown, but only between the axial ribs. Aperture white, inside faintly showing the outside bands if present.

Reticunassa visayaensis $\mathrm{sp}$. nov. and $R$. poppeorum sp. nov. have a multispiral protoconch, whereas the protoconch of $R$. goliath sp. nov. is paucispiral.

The strong columellar callus, together with the thickening of the inside of the outer lip and the strong varix, gives the aperture a round, "open mouth" appearance, whereas the other species in this group have a more oval-shaped aperture.

$R$. goliath sp. nov. and $R$. tringa both have a paucispiral protoconch, but the former has a considerably heavier and larger shell. It is similar to $R$. tringa, $R$. visayaensis sp. nov. and $R$. poppeorum sp. nov. in having weak spiral cords. $R$. goliath sp. nov. differs from $R$. paupera in weight, in its larger size, in the number of whorls, and in the size of the aperture relative to the total shell length.

\section{Habitat}

Sublittoral to $0-40 \mathrm{~m}$, frequently between 10 and $20 \mathrm{~m}$.

\section{Distribution}

Western Pacific; Indonesia (Papua), Australia (Queensland), Vanuatu, New Caledonia, and Loyalty Islands (Fig. 10).

\section{Reticunassa intrudens sp. nov. urn:1sid:zoobank.org:act:C79E7864-DE80-4A17-94BC-54932F8532CA}

Fig. 8I-L, 11; Table 2

\section{Etymology}

The name of this species refers to the spiral cords that seemingly intrude and continue over part of the body whorl of the protoconch.

\section{Type material}

\section{Holotype}

NEW CALEDONIA: MONTROUZIER 1993, stn 1260, Touho, Banc de Touho, 2044' S, $165^{\circ} 14^{\prime}$ E, 49-59 m, Sep. 1993 (MNHN IM-2000-22726, length 7.9 mm, width $4.3 \mathrm{~mm}$ ).

\section{Paratypes}

NEW CALEDONIA: same collection data as holotype (MNHN IM-2000-22722, MNHN IM-200028406, MNHN IM-2000-28407, 3 spm; ZMA.Moll.4.09.051, 1 spm; HD 35302, 3 spm; HK 196.01, $4 \mathrm{spm})$.

\section{Other material examined}

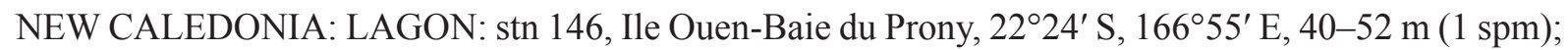
stn 357, Grand Récif Sud, $22^{\circ} 30^{\prime} \mathrm{S}, 167^{\circ} 07^{\prime} \mathrm{E}, 77 \mathrm{~m}$ (1 spm); stn 603, Yaté, $22^{\circ} 16^{\prime} \mathrm{S}, 167^{\circ} 05^{\prime} \mathrm{E}, 78-80 \mathrm{~m}$

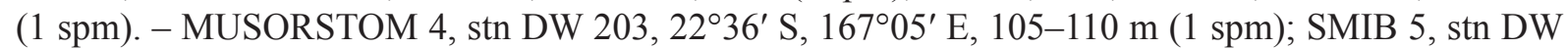
81, S New Caledonia, $22^{\circ} 38^{\prime} \mathrm{S}, 167^{\circ} 35^{\prime} \mathrm{E}, 110 \mathrm{~m}$ (5 spm); BATHUS 1, stn DW 678, W coast, $20^{\circ} 49^{\prime} \mathrm{S}$, $165^{\circ} 19^{\prime}$ E, 94-100 m (7 spm). - MONTROUZIER 1993, Touho, stn 1237, Touho Bay, 2046.9' S, $165^{\circ} 13.8^{\prime} \mathrm{E}, 0-1 \mathrm{~m}(5 \mathrm{spm})$; Touho, stn 1249 , Passe de Touho, $20^{\circ} 49^{\prime} \mathrm{S}, 165^{\circ} 19^{\prime} \mathrm{E}, 80-140 \mathrm{~m}$ (3 spm); Touho, stn $1250,20^{\circ} 46.7^{\prime} \mathrm{S}, 165^{\circ} 13.7^{\prime} \mathrm{E}, 3-6 \mathrm{~m}$ (7 spm); Touho, stn 1251, 2046. $0^{\prime}-20^{\circ} 46.5^{\prime} \mathrm{S}$, 


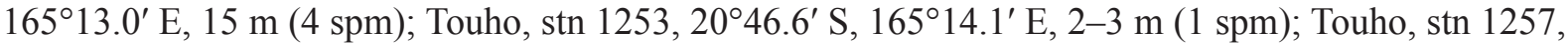

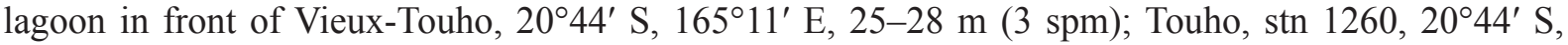
$165^{\circ} 14^{\prime}$ E, 49-59 m (70 spm); Touho, stn 1261, 20 $46^{\prime}-20^{\circ} 47^{\prime} \mathrm{S}, 165^{\circ} 15^{\prime}-165^{\circ} 16.5^{\prime} \mathrm{E}, 45-56 \mathrm{~m}(33$ spm); Touho, stn $1268,20^{\circ} 45.2^{\prime} \mathrm{S}, 165^{\circ} 08.0^{\prime} \mathrm{E}, 9-11 \mathrm{~m}$ (1 spm); Touho, stn $1275,20^{\circ} 49^{\prime} \mathrm{S}, 165^{\circ} 17^{\prime} \mathrm{E}$, $50-62 \mathrm{~m}(1 \mathrm{spm})$.

\section{Description}

\section{Holotype}

Protoconch. Bulbous, flattened, paucispiral, 1.75 whorls, yellowish, axially striate; separation between protoconch and teleoconch indistinct, last half whorl appears to have been "intruded" by the 5 spiral cords on first whorl of teleoconch.

SHELl. Teleoconch consisting of 5 strongly impressed whorls. Penultimate whorl and body whorl with 13 pronounced strong, round axial ribs, varix strong.

SPIRAL CORDS. Penultimate whorl with 7 and body whorl with 10 narrow, fine, equally wide spiral cords.

InTERCORDAL SCULPTURE. Minute spiral striae, occasionally also located on spiral cords.

APERTURE. Outer lip with 5 short and low lirate denticles. Columella wrinkled, columellar callus margined, elevated anteriorly and somewhat curved towards fasciole, posteriorly slightly extending onto body whorl. Parietal denticle prominent, anal canal rather wide.

OperCulum. Unknown.

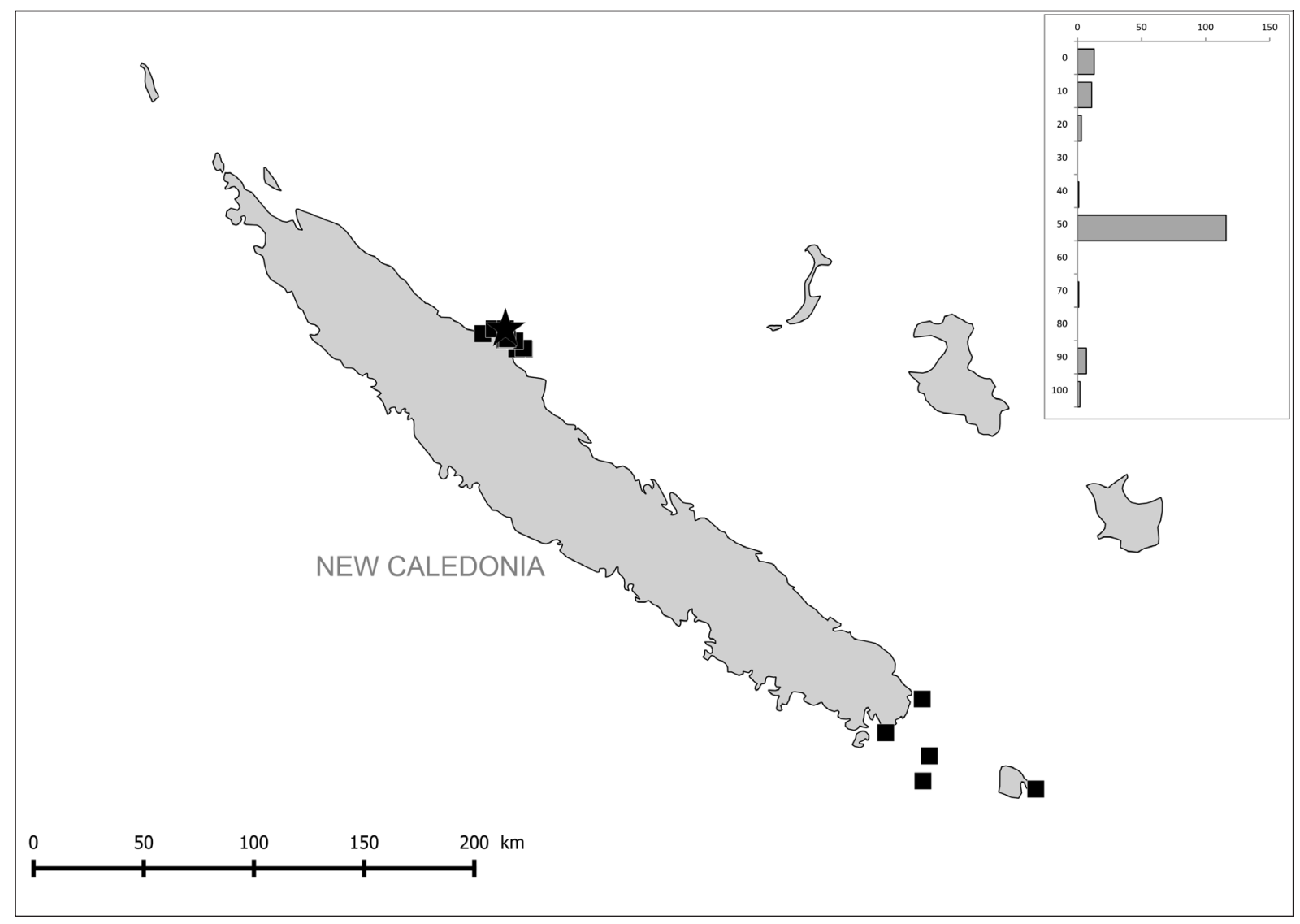

Fig. 11. Geographical and bathymetrical distribution of Reticunassa intrudens sp. nov. Star indicates type locality. Each bar represents all lv or dd specimens. 
SiPHONAL CANAL. Siphonal area with 1 stronger and 4 weak cords. Fasciole strong.

Color. Creamy-yellowish, some spiral cords darker. Whorls with light subsutural band, body whorl with 3 bands, darkest on the thick varix and at base of shell.

Adult SIZE. $5.4-9.7 \mathrm{~mm}$, usually $7.5-8.5 \mathrm{~mm}$.

\section{Remarks}

The most characteristic feature of this species is the broad, flattened, paucispiral and axially striated protoconch with, on the last part of it, 1-5 spiral cords that intrude from the teleoconch (Fig. 8L). This character was also mentioned by Cernohorsky (1984) in his description of Nassarius (Hima) pauperus (Gould, 1850) and was treated as intraspecific variation. These features, the broad, flat paucispiral protoconch and the extremely fine intercordal spiral striae of Reticunassa intrudens sp. nov., make it easy to distinguish it from $R$. paupera and the other species described in this paper. Moreover, $R$. intrudens sp. nov. lacks the minute pustules present in some other species described herein and most of the others also have a wider distribution; $R$. intrudens sp. nov. is only known from New Caledonia. It is one of the smallest species within this group.

\section{Habitat}

0-110 m in muddy sand, with the largest concentration of specimens collected at $45-60 \mathrm{~m}$.

\section{Distribution}

Only known from New Caledonia (Fig. 11).

Reticunassa thailandensis sp. nov. urn:1sid:zoobank.org:act:E0731396-9A77-4C1C-99F7-24B72E5B55DD

Fig. $8 \mathrm{M}-\mathrm{P}, 12$; Table 2

\section{Etymology}

This species is named after Thailand, the type locality.

\section{Type material}

\section{Holotype}

THAILAND: SE coast, Gulf of Thailand, Pattani Province, Ko Lopi, $06^{\circ} 40.313^{\prime}$ N, $101^{\circ} 43.852^{\prime}$ E, scuba diving, 3-15 m, leg. R.G. Moolenbeek and H. Dekker, 2003 (ZMA.Moll.4.09.047, length 7.35 $\mathrm{mm}$, width $3.8 \mathrm{~mm}$ ).

\section{Paratypes}

THAILAND: same collection data as for holotype (ZMA.Moll.4.09.048, $222 \mathrm{spm}$; 5 spm; MNHN IM2000-32481, 10 spm; GH, 2 spm; HD 24145, 6 spm; HK 139.01, 6 spm); Gulf of Thailand, Rayong Province, Talengam, near Suan Son, E of Ban Phe beach, leg. O.K. McCausland (HK 139.03, 1 spm).

\section{Other material examined}

THAILAND: Gulf of Thailand: type locality (4 spm); Ban Pak Nam Sakom, local fishery, $06^{\circ} 57^{\prime} \mathrm{N}$, 10049.36' E (ZMA.Moll.4.09.062, 3 spm; HD 1789, 6 spm); Ban Pak Nam Sakom, near Leela Resort, 06 $6^{\circ} 57.4^{\prime} \mathrm{N}, 100^{\circ} 51.6^{\prime} \mathrm{E}$, muddy pools (ZMA, 3 spm; HD 1190, $6 \mathrm{spm}$ ); Ban Talokapo, 06 $54.0^{\prime} \mathrm{N}$, 1012․ $24^{\prime}$ E, local fishery (HD 2146, 3 spm); Trat Province, Ko Chang, beach (HD 2793, 1 spm); Ko Samui, NE side of Chaweng Beach, 09 $32.6^{\prime} \mathrm{N}, 100^{\circ} 05.7^{\prime} \mathrm{E}$, in seagrass (HD 9572, $5 \mathrm{spm}$ ); Ko Samui, Ban Bangkao, 09²5. 2' N, 99 59.7' E, sand flat (HD 9574, 1 spm); Ko Kham, 0658.4' N, 100 51.6' E, 
scuba diving, 3-5 m (ZMA.Moll.093866, 14 spm); Pattani, in shell grit (ZMA.Moll.098947, 2 spm); off Pattaya, Ko Lan, scuba diving, reef, 3-6 m, leg. R.G. Moolenbeek (ZMA.Moll.4.09.063, 12 spm).

SINGAPORE: Bedok River area of East Coast Park, beached, leg. Chan Sow Yan (HK 139.04, 6 spm).

MALAYSIA: E coast, South China Sea, 02 $38^{\prime} 11^{\prime \prime} \mathrm{N}, 105^{\circ} 13^{\prime} 30^{\prime \prime} \mathrm{E}, 30-35 \mathrm{~m}$, leg. H. Morrison (GH, 2 spm; HK 139.02, 3 spm); South China Sea, on sand near wreck, $0^{\circ} 45^{\prime} 15^{\prime \prime}$ N, $105^{\circ} 36^{\prime} 11^{\prime \prime}$ E, leg. H. Morrison (GH, $1 \mathrm{spm})$.

INDONESIA: Laboratorium Onderzoek der Zee, 1938, stn Bat38/07a, Bay of Jakarta (ZMA. Moll.096217, 2 spm). - KEPULAUAN SERIBU 2005, stn Ser05/03, Java, Bay of Jakarta, Damar Kecil, 5 59'00" S, 106 50'43" E, scuba diving, 5-15 m (ZMA.Moll.175169, 7 spm); stn ser05/07,

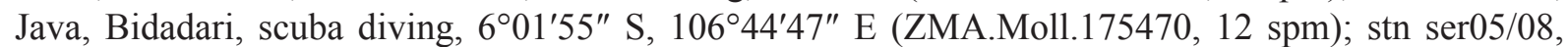
Java, Ayer Besar, 6 ${ }^{\circ} 00^{\prime} 06^{\prime \prime}$ S, 106 46'48" E (ZMA.Moll.154506, 1 spm); stn ser05/09, Java, Ubi Besar, scuba diving, 559'55" S, 1064'23" E (ZMA.Moll.175172, 8 spm); stn ser05/10, Java, Kelor, scuba diving, 5'51'00" S, 106 35'05" E, 5-35 m (ZMA.Moll.175173, 3 spm). - Java, W coast, Tjilintjing (ZMA.Moll.096215, ex coll. Butot 12405, 1 spm); Flores, E coast, near Larantuka, between Weri and Waibalun, leg. Fr.M. Vianney (ZMA.Moll.096211, 1 spm); Kalimantan, South China Sea, Pejantan I., $0^{\circ} 30^{\prime} 48.8^{\prime \prime} \mathrm{N}, 107^{\circ} 13^{\prime} 24^{\prime \prime} \mathrm{E}, 10-20 \mathrm{~m}$, leg. H. Morrison (GH, $5 \mathrm{spm}$ ); NETHERLANDS BERAU 2003, stn ber03/38, Berau Is, Maratua, 10-25 m (ZMA.Moll.175049, 1 spm).

PHILIPPINES: Palawan, Balabac I. (RMNH, 5 spm; 5 spm; HD 24144, 20 spm; GH, 6 spm; HK 139.05, 140 spm; coll. L. van Gemert, Zeist, the Netherlands, 20 spm; AMD, >300 spm); Olango I., Ziczac Point, 10-20 m, 2010 (HD 33413, 1 spm); Mindanao, Surigao del Norte, Basul I., 80-90 m, 2013 (HD $33414,1 \mathrm{spm})$.

\section{Description}

\section{Holotype}

Protoconch. Bulbous, flattened, paucispiral, 1.3 whorls, whitish, part of body whorl with growth lines.

SHELL. Teleoconch consisting of 4.5 impressed whorls. Axial ribs pronounced, rounded, 14 on penultimate and 13 on body whorl. Varix strong and broad.

SPIRAL CORDS. Continuous, narrow, thread-like spiral cords, increasing in height where they cross over axial ribs; 6 on penultimate and approximately 9 on body whorl. Spiral cord on periphery, the two above it, and the three at the base of body whorl more distinct; intercordal distances unequal.

INTERCORDAL SCULPTURE. Fine, distinct spiral striae, number depending on distance between spiral cords.

APERTURE. Oval, outer lip with 8 unequal denticles, one near periphery strongest. Columellar callus well bordered, slightly extending over body whorl; entire lip with short lirae. Parietal denticle strong.

Operculum. Yellowish, finely serrated.

SipHonal CANal. Narrow; fasciole strong. Siphonal area with approximately 4 cords; parietal denticle strong, anal canal wide.

CoLor. Yellowish-orange, penultimate whorl with two, body whorl with three orange-brown bands.

Adult Size. 5.5-9.1 mm, usually 7-8 $\mathrm{mm}$. One of the smaller species of the group. 


\section{Remarks}

The color is variable. Well-preserved specimens are cream or orange to light brown, either unicolor or with several darker-colored spiral cords. Occasionally remnants of bands are only present on the varix and on the fasciole.

The main difference between Reticunassa intrudens sp. nov and $R$. thailandensis sp. nov. is to be found in the protoconch morphology: the former has a protoconch with axial striae, in the latter it is partly smooth. Both are paucispiral and bulbous, and lack the microscopic pustules found in the other species described in this paper. $R$. paupera (Gould, 1850), $R$. visayaensis sp. nov. and $R$. poppeorum sp. nov. have a multispiral protoconch. $R$. thailandensis sp. nov. has more well developed spiral cords than the other species described here. Furthermore, in some specimens the 2-3 most anterior spiral cords are more strongly developed. In general, the distribution of the species, as shown on Fig. 12, might help with the identification.

\section{Habitat}

This species lives in sand and between dead corals, in depths of 1-90 m, usually found between 15 and $20 \mathrm{~m}$.

\section{Distribution}

Thailand, Singapore, Malaysia, Indonesia, and the Philippines, where it is common at Balabac I., but apparently rare in the central Visayas (Fig. 12).

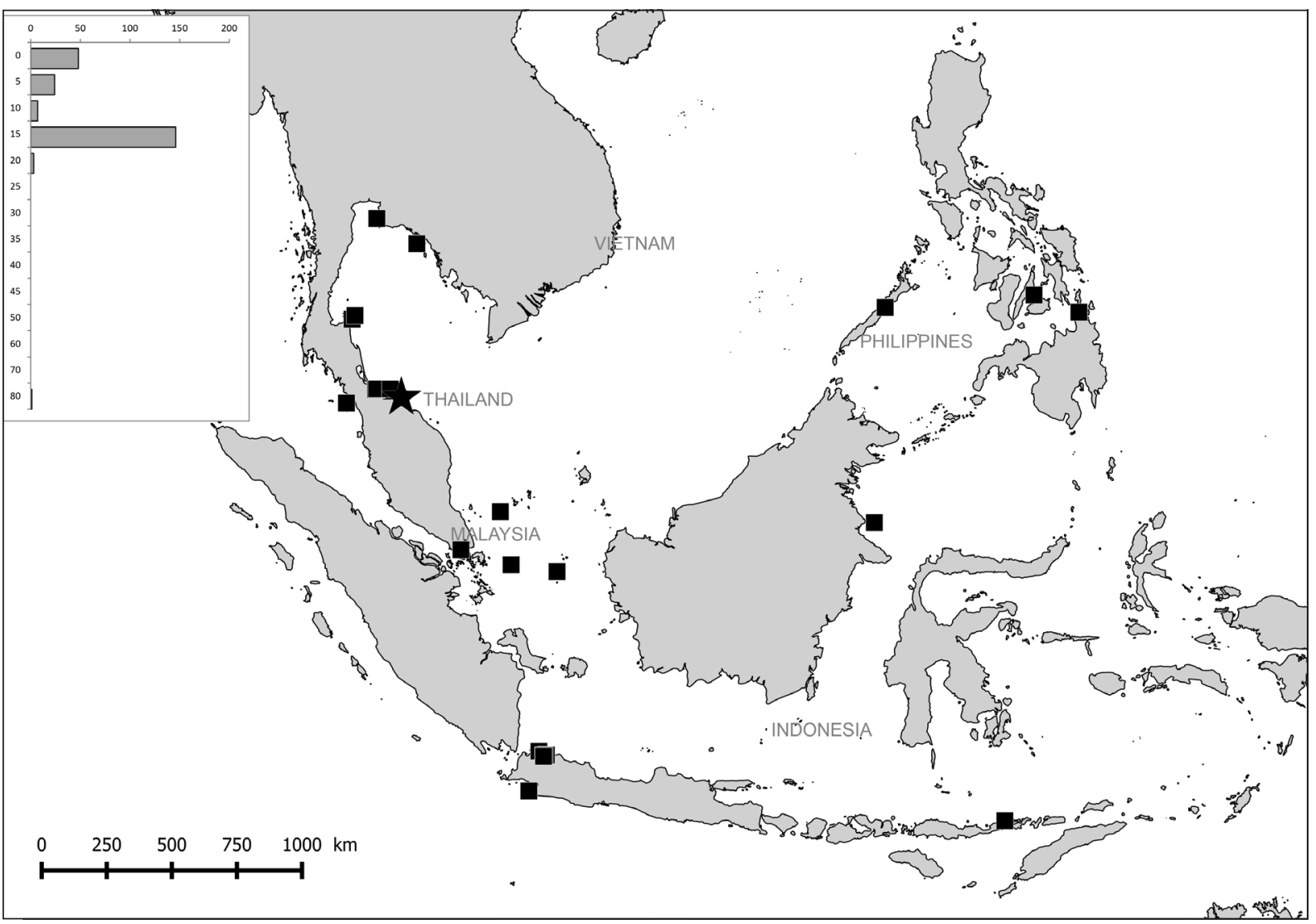

Fig. 12. Geographical and bathymetrical distribution of Reticunassa thailandensis sp. nov. Star indicates type locality. Each bar represents all lv or dd specimens. 


\section{Discussion}

This paper presents the first review on the diversification of genus Reticunassa from a molecular perspective. An integrative approach permitted the match between DNA sequences and morphological features to define the taxa discussed here. Previous references to "Nassarius pauperus" must be treated with caution because other species of Reticunassa might remain masked under that concept. This problem is particularly challenging when working with collections where specimens are kept dry and protoconchs have been lost, considering the small morphological interspecific differences among the species of Reticunassa.

So far, the only available morphological characters to distinguish species of the genus are the features of the protoconch, the spiral cords, the intercordal surface and the columella. Table 2 is a comparison of all available characters to facilitate species identification. However, in this paper we provide COI sequences that could help future identification of molecular samples. Reliably identified hologenophores for previously described species are becoming accessible, with their $C O I$ sequences available in online public libraries. For our new species we have prioritized the choice of name-bearing types such that they are also hologenophores, whenever possible, in order to avoid the perpetuation of a molluscan taxonomy in which many type specimens do not fulfill their function as name bearers (Bouchet \& Strong 2010).

As can be seen in Fig. 1A-B, the trees are congruent with our taxonomic hypotheses concerning the status of Reticunassa and the new species described in this paper, initially based on morphological observations. Our results are also consistent with what has been observed in other nassariids: the intraspecific variability is about $1.9 \%$ among species of Nassarius (Kool \& Galindo 2014) and ranges from $1.1 \%$ to $2.5 \%$ within species of Tritia (Couceiro et al. 2012; Couceiro et al. 2007; Simon-Bouhet et al. 2006). In general, K2P genetic distances between gastropod species span from $4.2 \%$ to $5.9 \%$ (Li et al. 2010; Kantor et al. 2012; Fedosov et al. 2014; Lorenz \& Puillandre 2015).

The absence of a discrete barcode gap in a dataset can be explained many ways. In this case, it can be related to poor intraspecific replication within some species, i.e., there is only one sequence each representing $R$. paupera and $R$. tringa.

The trees (Fig. 1A-B) reveal the existence of several lineages, with, so far, no morphologically recognizable characters (i.e., MNHN IM-2007-31778 and MNHN IM-2013-17562), preventing their description. Further studies on Reticunassa might disclose patterns to help assign names to those taxonomic units.

In this paper, no particular taxonomic ranking has been given to the two subclades found within Reticunassa. However, there are clear differences among them. SC1 is formed by species that lack spiral striae; instead, they have either axial striae ( $R$. hanraveni and $R$. silvardi) or relatively smooth intercordal surfaces (R. compacta, $R$. rotunda). Species of SC1 are distributed from the western Pacific Ocean to the Marquesas Islands. On the contrary, species of SC2 show the presence of spiral striae on the intercordal surface, and they occur in the Indian Ocean and the western Pacific Ocean. This result supports Galindo et al.'s (2016) hypothesis that geographical distribution patterns play an important role in the diversification of Nassariidae.

Kool \& Dekker (2006) placed Nassarius gregarius (Grabau \& King, 1928) in the N. pauperus complex, because it is similar in size and shape and had been treated as a synonym of $N$. pauperus by Cernohorsky (1984). However, because this species has a keeled protoconch, it should not be included in Reticunassa, but should continue to be included in the genus Nassarius. 
An integrative approach to taxonomy with all available data permits us to test the monophyly and the relationships of the species within the Nassarius pauperus complex, to reconstruct its taxonomic history and to present a comprehensive discussion of morphological features that are informative in species descrimination. Our results allow us to confer full generic status to the clade Reticunassa, which now includes 20 Recent species from the Indo-Pacific region.

\section{Acknowledgements}

As sources of material used in this paper, we want to highlight in particular the following expeditions: MONTROUZIER 1993 to New Caledonia; PANGLAO 2004 to the Philippines; SANTO 2006 to Vanuatu; and PAPUA NIUGINI to the Madang District of Papua New Guinea. They were sponsored by the Total Foundation, the Stavros Niarchos Foundation, the Prince Albert II of Monaco Foundation, Entrepose Contracting and Fondation EDF. In the field, Y. Kantor, N. Puillandre, E.E. Strong and M.C. Boisselier specifically preserved mollusk samples for molecular sequencing. Another source of sample material is the series of cruises to New Caledonia, Fiji, Tonga and the Solomon Island as part of the Tropical Deep-Sea Benthos programme (Bouchet et al. 2008), and also Bertrand Richer de Forges' Programme Lagon (material collected by Richer de Forges in 1995). We are grateful to P. Bouchet for placing the nassariid material from these sources at our disposal. Thanks to V. Héros, P. Maestrati and B. Buge for their highly appreciated support and comments. We are much indebted to the following persons for the loan of material, providing information, supplying literature and allowing us access to the collections in their care: J. Goud and R.G. Moolenbeek, both NBC, G.M. Hansen, D. Massemin and P. Stahlschmidt. Special thanks to L. Charles (Musée d'Histoire Naturelle de Bordeaux) for providing the $R$. tringa type series. R.H. Cowei, R.G. Moolenbeek and S.P. Kool reviewed the text and gave very valuable remarks. P. Bouchet guided us through many taxonomic and nomenclatural decisions. M.A. Alonso Zarazaga (International Commission on Zoological Nomenclature) helped us with the Latin declensions. G. Marani built and has managed the Geographic Information System for the biodiversity of Nassariidae. M. Caballer is thanked for photographing the holotypes as part of the E-RECOLNAT ANR-11INBS-0004, MNHN program and S. Pont (SEM-EDS) for securing the electronic microscopic images. This project was supported by the "Bibliothèque du Vivant" network, funded by the CNRS-MNHNINRA-CEA (Genoscope), the 'Service de Systématique Moléculaire' (UMS 2700 CNRS-MNHN), and the deep-sea studies developed in the French-Taiwanese TF-DeepEvo ANR-NSC project (ANR 12ISV7-0005-01). Thanks to B.A. Marshall and an anonymous reviewer for their comments, which helped to improve the manuscript.

\section{References}

Adams C.B. 1845. Specierum novarum conchyliorum, in Jamaica repertorum, synopsis. Proceedings of the Boston Society of Natural History 2: 1-17.

Adams H. \& Adams A. 1853-1858. The Genera of Recent Mollusca; Arranged According to their Organization. London, van Voorst. Vol. 1: xl +484 pp.; vol. 2: 661 pp.; vol. 3: 138 pls. [Published in parts: Vol. 1: i-xl (1858), 1-256 (1853), 257-484 (1854). Vol. 2: 1-92 (1854), 93-284 (1855), 285-412 (1856), 413-540 (1857), 541-661 (1858). Vol. 3: pl. 1-32 (1853), 33-96 (1855), 97-112 (1856), 113128 (1857), 129-138 (1858).]

Bouchet P. \& Strong E.E. 2010. Historical name-bearing types in marine molluscs: an impediment to biodiversity studies? In: Polaszek A. (ed.) Systema Naturae 250 - The Linnaean Ark: 63-74. CRC Press, Boca Raton, LA.

Bouchet P., Héros V., Lozouet P. \& Maestrati P. 2008. A quarter-century of deep-sea malacological exploration in the South and West Pacific: Where do we stand? How far to go? In: Héros V., Cowie R.H. \& Bouchet P. (eds) Tropical Deep-Sea Benthos, vol. 25: 9-40. Mémoires du Muséum national d'Histoire naturelle 196. 
GALINDO L.A. et al., The Nassarius pauperus complex, part 3

Bozzetti L. 2006. Nassarius (Hima) priscardi (Gastropoda: Prosobranchia: Nassariidae) nuova specie dal Madagascar Meridionale. Malacologia Mostra Mondiale 53: 17.

Cernohorsky W.O. 1972. Indo-Pacific Nassariidae (Mollusca: Gastropoda). Records of the Auckland Institute and Museum 9: 125-194.

Cernohorsky W.O. 1974. Remarks on the taxonomy of Japanese Nassariidae. Venus 33 (2): 51-64.

Cernohorsky W.O. 1984. Systematics of the family Nassariidae (Mollusca: Gastropoda). Bulletin of the Auckland Institute and Museum 14: 1-356.

Chisholm L.A., Morgan J.A., Adlard R.D. \& Whittington I.D. 2001. Phylogenetic analysis of the Monocotylidae (Monogenea) inferred from 28S rDNA sequences. International Journal for Parasitology 31: 1537-1547. http://dx.doi.org/10.1016/S0020-7519(01)00313-7

Cossmann M. 1901. Essais de Paléoconchologie Comparée. Fourth edition. The author and Société d'Éditions Scientifiques, Paris.

Couceiro L., Barreiro R., Ruiz J.M. \& Sotka E.E. 2007. Genetic isolation by distance among populations of the netted dog whelk Nassarius reticulatus (L.) along the European Atlantic coastline. Journal of Heredity 98: 603-610. http://dx.doi.org/10.1093/jhered/esm067

Couceiro L., López L., Sotka E.E., Ruiz J.M. \& Barreiro R. 2012. Molecular data delineate cryptic Nassarius species and characterize spatial genetic structure of N. nitidus. Journal of the Marine Biological Association of the United Kingdom 92: 1175-1182. http://dx.doi.org/10.1017/S0025315411000816

Fedosov A., Olivera B.M., Watkins M. \& Barkalova V. 2014. A new species of Casmaria H. Adams \& A. Adams, 1853 (Gastropoda, Cassidae) from the Philippines identified by molecular data. European Journal of Taxonomy 78: 1-13. http://dx.doi.org/10.5852/ejt.2014.78

Folmer O., Black M., Hoeh W., Lutz R. \& Vrijenhoek R. 1994. DNA primers for amplification of mitochondrial cytochrome c oxidase subunit 1 from diverse metazoan invertebrates. Molecular Marine Biology and Biotechnology 3: 294-299.

Galindo L.A., Puillandre N., Strong E.E. \& Bouchet P. 2014. Using microwaves to prepare gastropods for DNA barcoding. Molecular Ecology Resources 14: 700-705. http://dx.doi.org/10.1111/1755$\underline{0998.12231}$

Galindo L.A., Puillandre N., Utge J., Lozouet P. \& Bouchet P. 2016. The phylogeny and systematics of the Nassariidae revisited (Gastropoda, Buccinoidea). Molecular Phylogenetics and Evolution 99: 337-353. http://dx.doi.org/10.1016/j.ympev.2016.03.019

Gould A.A. 1850. Descriptions with drawings of several new species of shells brought home from the United States Exploring Expedition. Proceedings of the Boston Society of Natural History 3: 151-156, 169-172.

Gould A.A. 1852. United States Exploring Expedition during the Years 1838-1842 under the Command of Charles Wilkes, U.S.N. Volume 12. Mollusca and Shells. Gould \& Lincoln, Boston. Available from http://biodiversitylibrary.org/page/10991152 [accessed 21 Dec. 2016].

Gould A.A. 1862. Otia Conchologia: Descriptions of Shells and Mollusks from 1839 to 1862. Gould \& Lincoln, Boston.

Grabau A.W. \& King S.G. 1928. Peking Society of Natural History, Educational Handbooks. No. 2: Shells of Peitaiho. Peking Society of Natural History, Beijing.

Gray J.E. 1847a. The classification of the British Mollusca by W.E. Leach, M.D. Annals and Magazine of Natural History 20 (133): 267-273. http://dx.doi.org/10.1080/037454809496042 
Gray J.E. 1847b. A list of the genera of recent Mollusca, their synonyma and types. Proceedings of the Zoological Society of London 15: 129-219. Available from http://biodiversitylibrary.org/page/12862913 [accessed 21 Dec. 2016].

Gray J.E. 1852. Molluscorum Britanniae. Synopsis of the Mollusca of Great Britain. John van Vorst, London.

Iredale T. 1916. On two editions of Dumeril's Zoologie Analytique. Proceedings of the Malacological Society of London 12: 79-84.

Iredale T. 1936. Australian molluscan notes. No. 2. Records of the Australian Museum 19: 267-340. http://dx.doi.org/10.3853/j.0067-1975.19.1936.704

Johnson R.I. 1964. The recent Mollusca of Augustus Addison Gould. United States National Museum Bulletin 239: 1-182. Available from http://biodiversitylibrary.org/page/7892491 [accessed 21 Dec. 2016].

Kantor Y., Puillandre N., Rivasseau A. \& Bouchet P. 2012. Neither a buccinid nor a turrid: a new family of deep-sea snails for Belomitra P. Fischer, 1883 (Mollusca, Neogastropoda), with a review of Recent Indo-Pacific species. Zootaxa 3496: 1-64.

Kool H.H. \& Dekker H. 2006. Review of the Nassarius pauper (Gould, 1850) complex (Gastropoda Nassariidae). Part 1, with the description of four new species from the Indo-West Pacific. Visaya 1 (6): $54-75$.

Kool H.H. \& Dekker H. 2007. Review of the Nassarius pauper (Gould, 1850) complex (Gastropoda, Nassariidae). Part 2, the Western Indian Ocean species, with the description of two new species and introducing a nomen novum. Visaya 2 (2): 62-77.

Kool H.H. \& Galindo L.A. 2014. Description and molecular characterization of six new species of Nassarius (Gastropoda, Nassariidae) from the Western Pacific Ocean. American Malacological Bulletin 32: 147-164. http://dx.doi.org/10.4003/006.032.0202

Lee H.G. 2013. What to do with Hima of authors, not of Leach, 1852? Shell-O-Gram 54 (2): 3-5.

Li H., Lin D., Fang H., Zhu A. \& Gao Y. 2010. Species identification and phylogenetic analysis of genus Nassarius (Nassariidae) based on mitochondrial genes. Chinese Journal of Oceanology and Limnology 28: 565-572. http://dx.doi.org/10.1007/s00343-010-9031-4

Lorenz F. \& Puillandre N. 2015. Conus hughmorrisoni, a new species of cone snail from New Ireland (Gastropoda: Conidae). European Journal of Taxonomy 129: 1-15. http://dx.doi.org/10.5852/ ejt.2015.129

Martin J.C. 2008. Nassariidae. In: Poppe G.T. (ed.) Philippine Marine Mollusks, Volume 2 (Gastropoda-Part 2): 114-129. ConchBooks, Hackenheim, Germany.

Miller M.A., Pfeiffer W. \& Schwartz T. 2010. Creating the CIPRES Science Gateway for inference of large phylogenetic trees. In: Proceedings of the Gateway Computing Environments Workshop (GCE): $1-8$. New Orleans.

Pleijel F., Jondelius U., Norlinder E., Nygren A., Oxelman B., Schander C., Sundberg P. \& Thollesson M. 2008. Phylogenies without roots? A plea for the use of vouchers in molecular phylogenetic studies. Molecular Phylogenetics and Evolution 48: 369-371. http://dx.doi.org/10.1016/j.ympev.2008.03.024

Preston H.B. 1907. Description of two new species of Nassa from Fiji and New Caledonia. Journal de Conchyliologie 54: 267-269.

Puillandre N., Bouchet P., Boisselier-Dubayle M.-C., Brisset J., Buge B., Castelin M., Chagnoux S., Christophe T., Corbari L., Lambourdière J., Lozouet P., Marani G., Rivasseau A., Silva N., Terryn Y., 
GALINDO L.A. et al., The Nassarius pauperus complex, part 3

Tillier S., Utge J. \& Samadi S. 2012a. New taxonomy and old collections: integrating DNA barcoding into the collection curation process: DNA barcoding in museums. Molecular Ecology Resourses 12: 396-402. http://dx.doi.org/10.1111/j.1755-0998.2011.03105.x

Puillandre N., Lambert A., Brouillet S. \& Achaz G. 2012b. ABGD, Automatic Barcode Gap Discovery for primary species delimitation. Molecular Ecology 21 (8): 1864-1877. http://dx.doi.org/10.1111/ j.1365-294X.2011.05239.x

Richard G.R., Tröndle J. \& Salvat B. 1984. Nassariidae de Polynésie Française. Xenophora 21: 7-17.

Risso A. 1826. Histoire naturelle des principales Productions de l'Europe méridionale et particulièrement de celles des Environs de Nice et des Alpes Maritimes, IV. F.G. Levrault, Paris.

Ronquist F. \& Huelsenbeck J.P. 2003. MrBayes 3: Bayesian phylogenetic inference under mixed models. Bioinformatics 19: 1572-1574. http://dx.doi.org/10.1093/bioinformatics/btg180

Shuto T. 1969. Neogene gastropods from Panay I., the Philippines. Memoirs of the Faculty of Science, Kyushu University, Geological Series 19: 1-250.

Simon-Bouhet B., Garcia-Meunier P. \& Viard F. 2006. Multiple introductions promote range expansion of the mollusc Cyclope neritea (Nassariidae) in France: evidence from mitochondrial sequence data. Molecular Ecology 15: 1699-1711. http://dx.doi.org/10.1111/j.1365-294X.2006.02881.x

Souverbie M. \& Montrouzier R.P. 1864. Descriptions d'espèces nouvelles de l'Archipel Calédonien. Journal de Conchyliologie 12: 261-275.

Strøm H. 1768. Beskrivelse over norske Insecter. Det Kongelige Norske Videnskabers Selskabs Skrifter 4: 313-371.

Tamura K., Peterson A., Peterson N., Stecher G., Nei M. \& Kumar S. 2011. MEGA5: Molecular Evolutionary Genetics Analysis using maximum likelihood, evolutionary distance, and maximum parsimony methods. Molecular Biology and Evolution 28: 2731-2739. http://dx.doi.org/10.1093/ $\underline{\mathrm{molbev} / \mathrm{msr} 121}$

Von Maltzan H. 1884. Diagnosen neuer Senegambischer Gastropoden. Nachrichtsblatt der Deutschen Malakozoologischen Gesellschaft 16: 65-73.

Woodring W.P. 1928. Miocene mollusks from Bowden, Jamaica. Part II. Gastropods and discussion of results. Contributions to the geology and paleontology of the West Indies. Carnegie Institution of Washington Publication 385: 1-564.

Manuscript received: 1 February 2016

Manuscript accepted: 1 August 2016

Published on: 10 February 2017

Guest editors: Line Le Gall, Frédéric Delsuc, Stéphane Hourdez, Guillaume Lecointre

and Jean-Yves Rasplus

Desk editor: Danny Eibye-Jacobsen

Printed versions of all papers are also deposited in the libraries of the institutes that are members of the EJT consortium: Muséum national d'Histoire naturelle, Paris, France; Botanic Garden Meise, Belgium; Royal Museum for Central Africa, Tervuren, Belgium; Natural History Museum, London, United Kingdom; Royal Belgian Institute of Natural Sciences, Brussels, Belgium; Natural History Museum of Denmark, Copenhagen, Denmark; Naturalis Biodiversity Center, Leiden, the Netherlands. 